\title{
Forwarding strategies and optimal power allocation for coherent and noncoherent relay networks
}

\author{
Somak Datta Gupta \\ West Virginia University
}

Follow this and additional works at: https://researchrepository.wvu.edu/etd

\section{Recommended Citation}

Datta Gupta, Somak, "Forwarding strategies and optimal power allocation for coherent and noncoherent relay networks" (2006). Graduate Theses, Dissertations, and Problem Reports. 1849.

https://researchrepository.wvu.edu/etd/1849

This Thesis is protected by copyright and/or related rights. It has been brought to you by the The Research Repository @ WVU with permission from the rights-holder(s). You are free to use this Thesis in any way that is permitted by the copyright and related rights legislation that applies to your use. For other uses you must obtain permission from the rights-holder(s) directly, unless additional rights are indicated by a Creative Commons license in the record and/ or on the work itself. This Thesis has been accepted for inclusion in WVU Graduate Theses, Dissertations, and Problem Reports collection by an authorized administrator of The Research Repository @ WVU. For more information, please contact researchrepository@mail.wvu.edu. 


\title{
Forwarding Strategies and Optimal Power Allocation for Coherent and Noncoherent Relay Networks
}

\author{
by \\ Somak Datta Gupta \\ Thesis submitted to the \\ College of Engineering and Mineral Resources \\ at West Virginia University \\ in partial fulfillment of the requirements \\ for the degree of \\ Master of Science \\ in \\ Electrical Engineering \\ Daryl Reynolds, Ph.D., Chair \\ Arun Ross, Ph.D. \\ Matthew C.Valenti, Ph.D.
}

Lane Department of Computer Science and Electrical Engineering

Morgantown, West Virginia

2006

Keywords: Cooperative Diversity, Amplify and Forward, Decode and Forward, Power Optimization, Power Allocation, Preferred Relay Location, 


\begin{abstract}
Forwarding Strategies and Optimal Power Allocation for Coherent and Noncoherent Relay Networks

by

Somak Datta Gupta

Master of Science in Electrical Engineering

West Virginia University

Daryl Reynolds, Ph.D., Chair
\end{abstract}

In fading wireless channels, relays are used with the aim of achieving diversity and thus overall performance gain. In cooperative relay networks, various forwarding techniques like amplify and forward (AF) and decode and forward (DF) are used at the relay for better throughput and improved BER performance than traditional multihop systems. In a power constrained environment, the performance can be further improved by using an optimal power allocation strategy. The relative position of the relay with respect to the source and destination also has an immense effect on the efficacy of the relay.

We position the relay at various positions in a planar grid, with the position of source and destination being fixed, and we investigate the effect that the positioning of the relay has on a relaying system. We use our three terminal model to optimize the power allocation under total transmit power constraint, to maximize the instantaneous signal-to-noise ratio (SNR) at destination, and thus achieve improved throughput and BER performance, while using AF and DF protocols. We evaluate the performance of our system for both coherent and noncoherent modulation in a Rayleigh block fading channel. Quadrature phase shift keying (QPSK) is used in the coherent case and 4-Frequency shift keying (4-FSK) is used in the noncoherent case.

Previous works involving power allocation schemes have mainly concentrated on optimizing information theoretic quantities like capacity and outage probability. We derive expressions for instantaneous SNR using our model and optimize the power allocation based on that, with the final aim of achieving improved uncoded BER. Analytical expressions of the instantaneous SNR at the destination are derived for both AF and DF. These expressions are numerically optimized to obtain an optimum power allocation strategy for each position of the relay in both the AF and DF schemes using coherent or noncoherent detection.

We compare the performance of the AF and DF protocols based on their positional BER and throughput at different received SNR and notice that our power optimized schemes outperform existing power control schemes at certain areas. Finally we also identify the shape and area of the regions where relaying would provide performance gains for both the protocols at different received SNRs. 
To My Family 


\section{Acknowledgments}

Limited by my power of expression, I want to convey my sincere appreciation to all those people, past and present, who helped me come to this point and helped me during my stay at WVU. I am what I am because of them and would never forget them. First, I would thank my adviser, Dr. Daryl Reynolds for guiding me towards a goal. I am grateful for the freedom he allowed during my research, always supporting, encouraging and offering valuable advice. I have learnt the basics of research from him, ways to approach and tackle problems and also improved my writing skills. His drive for perfection is contagious and I am sure it would be an asset in the coming years. I would also like to thank him for his patience and support during difficult times.

I am thankful to Dr. Matthew Valenti for helping me to develop a scientific and logical outlook through his courses, seminars and discussions. It was a pleasure being his student. I would also express my sincere thanks to Dr. Arun Ross for being in my committee. I would like to thank Dr. Natalia Schmid and Dr. Brian Woerner for giving me a chance to study under their guidance. I must convey my sincere thanks to all WCRL members, specially Shi, Rohit, Ram and Kanchan for the numerous helpful discussions and also the lighter moments we shared. And I would fail my duties if I do not thank National Research Center for Coal and Energy for supporting me during my MS and my wonderful colleagues there.

I would like to express my heartfelt gratitude to my dear friends Abanti, Aniruddha, Saswata, Indrani and Sudeshnadi and family, for standing by during good times and bad and supporting me all these days. I have no words to express my regards to Indrani, who has been more than a sister to me, taking care, feeding and encouraging me all the time.

Finally I would thank my mother, Mamata Datta Gupta, father Sadhan Datta Gupta and brother, Sayan Datta Gupta, for believing in me and offering unflinching support and encouragement in all I did. Though they are continents away, their blessings and love is always there and are a constant source of inspiration. 


\section{Contents}

Acknowledgments iv

List of Figures vii

Notation $\quad$ xi

1 Introduction 1

2 Wireless Communication Systems and Relay Networks $\quad 7$

2.1 Linear Modulation and Optimal Detection in AWGN channels . . . . . . . . 7

2.2 BPSK in Additive White Gaussian Noise Channel . . . . . . . . . . . . . . . 11

2.3 QPSK in Additive White Gaussian Noise Channel . . . . . . . . . . . . . . . 12

2.4 Coherent PSK in Rayleigh Fading Channel . . . . . . . . . . . . . . . . . . . 13

2.5 FSK in Rayleigh Fading Channel . . . . . . . . . . . . . . . . . . . . . . . . 14

2.6 Noncoherent FSK in Rayleigh Block Fading Channel . . . . . . . . . . . . . 16

2.7 Diversity and Maximal Ratio Combining . . . . . . . . . . . . . . . . . 17

2.8 Path Loss Models . . . . . . . . . . . . . . . . . . . . . . . . . . 20

2.9 Relay Networks: An Overview . . . . . . . . . . . . . . . . . . . 22

3 Amplify and Forward with Coherent Modulation 24

3.1 System Model . . . . . . . . . . . . . . . . . . . . . . 24

3.2 Performance Improvement Offered by Amplify and Forward . . . . . . . . . 28

3.2.1 Power Optimization in Collinear Case . . . . . . . . . . . . . . . . . 29

3.2 .2 Power Optimization Over a Grid and Area of Interest . . . . . . . . . 34

3.3 Conclusion . . . . . . . . . . . . . . . . . . . 39

4 Decode and Forward with Coherent Modulation 40

4.1 Introduction . . . . . . . . . . . . . . . . . . . 40

4.2 Performance Improvement Offered by Decode and Forward . . . . . . . . . . 42

4.2.1 Power Optimization in Collinear Case . . . . . . . . . . . . . . . . 43

4.2 .2 Power Optimization Over a Grid and Area of Interest . . . . . . . . . 48

4.3 Performance Comparison of Amplify Forward and Decode Forward . . . . . 52

4.4 Conclusion . . . . . . . . . . . . . . . . . . 54 
5 Forwarding with Noncoherent Modulation $\quad 58$

5.1 Introduction . . . . . . . . . . . . . . . . . 58

5.2 Performance Improvement Offered by Amplify and Forward and Decode and Forward . . . . . . . . . . . . . . . . . . . . 60

5.2 .1 Amplify and Forward:Collinear Case . . . . . . . . . . . . 60

5.2 .2 Decode and Forward:Collinear Case . . . . . . . . . . . . . . 61

5.3 Power Optimization in Collinear Case . . . . . . . . . . . . . . . . . . 62

5.4 Power Optimization Over a Grid and Area of Interest . . . . . . . . . . . 67

5.5 Conclusion . . . . . . . . . . . . . . . . . . . . 72

6 Conclusions $\quad 73$

6.1 Summary and Conclusions . . . . . . . . . . . . . . . . . . . . . 73

6.2 Future Work . . . . . . . . . . . . . . . . . . . . . . . . . . . . 74

$\begin{array}{lr}\text { References } & 75\end{array}$

\begin{tabular}{lr}
\hline Appendix & 80
\end{tabular}

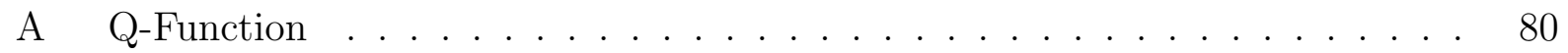

B Bernoulli Random Variable . . . . . . . . . . . . . . . . . . . . . . . . 81

C Cyclic Redundancy Check . . . . . . . . . . . . . . . . . . . . . . 82

D Quasi-Newton Method . . . . . . . . . . . . . . . . . . 83 


\section{List of Figures}

2.1 correlation-type demodulator. . . . . . . . . . . . . . . . . 9

2.2 BPSK constellation . . . . . . . . . . . . . . . . . . 11

2.3 QPSK constellation diagram with Gray coding. . . . . . . . . . . . . . . . . 13

2.4 Receiver structure for M-FSK with coherent modulation. . . . . . . . . . . . 15

2.5 BER curve with maximum ratio combining for BPSK modulation having varied number of diversity branches. . . . . . . . . . . . . . . . . . . 20

3.1 System model for a three terminal cooperative relay network. . . . . . . . . . 25

3.2 Analytical and simulation results for direct source to relay communication using distance model with received SNR of 10dB. The x-axis represents the transmitter to receiver distance. . . . . . . . . . . . . . . . . . . 27

3.3 Comparison of semi-analytical and simulated BER performance of AF . The transmit power is equally divided in both the phases. . . . . . . . . . . . . . 30

3.4 Comparison of semi-analytical BER performance of AF with various different power allocations in Phase 1 and Phase 2. . . . . . . . . . . . . . . . 31

3.5 Comparison of simulated BER performance of AF with various different power allocations in Phase 1 and Phase 2 and direct communication. . . . . . . . .

3.6 Comparison of simulated BER performance of AF with various different power allocations in Phase 1 and Phase 2, direct communication, and AF with optimal power allocation. . . . . . . . . . . . . . . . .

3.7 Comparison of simulated BER performance of AF with optimal power allocation and direct communication for received SNRs of $10 \mathrm{~dB}$ and $20 \mathrm{~dB}$. . . . . .

3.8 Comparison of the throughput of AF with optimal power allocation and direct communication for received SNRs of $10 \mathrm{~dB}$ and $20 \mathrm{~dB}$. . . . . . . . . . . . . . 34

3.9 Layout of the grid with the source at $\mathrm{x}, \mathrm{y}=(-0.5,0)$ and destination at $\mathrm{x}, \mathrm{y}=$ $(0.5,0) \ldots \ldots \ldots \ldots . \ldots \ldots \ldots$

3.10 Phase 1 power $p_{1}$ for AF . The received SNR is $10 \mathrm{~dB}$. Source at $\mathrm{x}, \mathrm{y}=(-0.5,0)$ and destination at $\mathrm{x}, \mathrm{y}=(0.5,0)$. The $\mathrm{z}$ axis represents the optimal value of $p_{1} .35$

3.11 BER performance of AF over the grid. The received SNR is 10dB. Source at

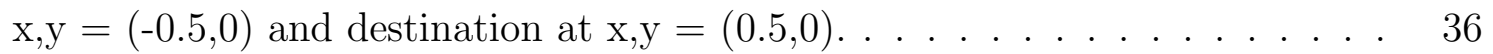

3.12 BER performance of AF and direct communication over the grid . The received SNR at destination is $10 \mathrm{~dB}$. Source at $\mathrm{x}, \mathrm{y}=(-0.5,0)$ and destination at $\mathrm{x}, \mathrm{y}=(0.5,0) \ldots \ldots \ldots \ldots \ldots$ 
3.13 The inner lighter area represents the region for relaying in AF. The received SNR is $10 d B$. Source at $\mathrm{x}, \mathrm{y}=(-0.5,0)$ and destination at $\mathrm{x}, \mathrm{y}=(0.5,0)$. . .

3.14 Comparison of BER performances of AF with received SNRs 10dB and 20dB. Source at $\mathrm{x}, \mathrm{y}=(-0.5,0)$ and destination at $\mathrm{x}, \mathrm{y}=(0.5,0) \ldots \ldots$. . . . .

3.15 The inner lighter areas represent the region for relaying in coherent environment using AF at the relay. The received SNR for the left hand plot is $10 \mathrm{~dB}$ and that for the right hand one is $20 \mathrm{~dB}$. Source at $\mathrm{x}, \mathrm{y}=(-0.5,0)$ and destination at $\mathrm{x}, \mathrm{y}=(0.5,0) \ldots \ldots \ldots \ldots$

4.1 BER performances of the system when the relay forwards any received data, does a CRC-5 check before forwarding and when it forwards only correct data from the relay. Equal power is allocated to both the transmission phases. The

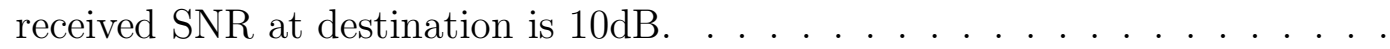

4.2 Comparison of semi-analytical and simulated BER performance of DF . The transmit power is equally divided in both the phases. . . . . . . . . . .

4.3 Comparison of semi-analytical BER performance of DF with various different power allocations in Phase 1 and Phase 2. . . . . . . . . . . . . . . . .

4.4 Comparison of simulated BER performance of DF with various different power allocations in Phase 1 and Phase 2 and direct communication. . . . . . . . .

4.5 Comparison of simulated BER performance of DF with various different power allocations in Phase 1 and Phase 2, direct communication, and DF with optimal power allocation. . . . . . . . . . . . . . . .

4.6 Comparison of simulated BER performance of DF with optimal power allocation and direct communication for received SNRs of $10 \mathrm{~dB}$ and $20 \mathrm{~dB}$. . . . .

4.7 Comparison of the throughput of DF with optimal power allocation and direct communication for received SNRs of $10 \mathrm{~dB}$ and $20 \mathrm{~dB}$. . . . . . . . . . . . . .

4.8 BER performance of DF over the grid. The received SNR is 10dB. Source at $\mathrm{x}, \mathrm{y}=(-0.5,0)$ and destination at $\mathrm{x}, \mathrm{y}=(0.5,0) \ldots \ldots . . . . . .$.

4.9 BER performances of DF over the grid and direct communication. The received SNR is $10 \mathrm{~dB}$. Source at $\mathrm{x}, \mathrm{y}=(-0.5,0)$ and destination at $\mathrm{x}, \mathrm{y}=(0.5,0)$.

4.10 The inner lighter area represents the region for relaying in DF. The received $\mathrm{SNR}$ is $10 \mathrm{~dB}$. Source at $\mathrm{x}, \mathrm{y}=(-0.5,0)$ and destination at $\mathrm{x}, \mathrm{y}=(0.5,0) . \ldots 50$

4.11 Phase 1 power $p_{1}$ for DF. The received SNR is $10 \mathrm{~dB}$. Source at $\mathrm{x}, \mathrm{y}=(-0.5,0)$ and destination at $\mathrm{x}, \mathrm{y}=(0.5,0)$. The $\mathrm{z}$ axis represents the optimal value of $p_{1} .50$

4.12 Comparison of BER performances of DF with received SNRs 10dB and 20dB. Source at $\mathrm{x}, \mathrm{y}=(-0.5,0)$ and destination at $\mathrm{x}, \mathrm{y}=(0.5,0) \ldots . . .$. . .

4.13 The inner lighter areas represent the region for relaying in coherent environment using DF at the relay. The received SNR for the left hand plot is $10 \mathrm{~dB}$ and that for the right hand one is $20 \mathrm{~dB}$. Source at $\mathrm{x}, \mathrm{y}=(-0.5,0)$ and destination at $\mathrm{x}, \mathrm{y}=(0.5,0) \ldots \ldots \ldots \ldots$

4.14 BER performance of AF with arbitrary and optimal power allocation. The received $\mathrm{SNR}$ is $10 \mathrm{~dB}$. Source at $\mathrm{x}, \mathrm{y}=(0,0)$ and destination at $\mathrm{x}, \mathrm{y}=(1,0)$. $\mathrm{AF}(10-90)$ represents the case when 10 percent of the total power is sent in Phase 1 and 90 percent in Phase 2 and so on. . . . . . . . . . . . . . 
4.15 BER performance of DF with arbitrary and optimal power allocation. The received $\mathrm{SNR}$ is $10 \mathrm{~dB}$. Source at $\mathrm{x}, \mathrm{y}=(0,0)$ and destination at $\mathrm{x}, \mathrm{y}=(1,0)$. $\mathrm{DF}(10-90)$ represents the case when 10 percent of the total power is sent in Phase 1 and 90 percent in Phase 2 and so on. . . . . . . . . . . . . . . .

4.16 BER performance of $\mathrm{AF}$ and $\mathrm{DF}$ with optimal power allocation and direct transmission. The received SNR is $10 \mathrm{~dB}$. Source at $\mathrm{x}, \mathrm{y}=(-0.5,0)$ and destination at $\mathrm{x}, \mathrm{y}=(0.5,0) \ldots \ldots \ldots \ldots$

4.17 Comparison of throughput performance between [Qi et.al.] and our model for $\mathrm{DF}$ and AF with optimal power allocation. The received SNR is 10dB. Source at $\mathrm{x}, \mathrm{y}=(0,0)$ and destination at $\mathrm{x}, \mathrm{y}=(1,0) \ldots \ldots$. . . . . . . . . . 55

4.18 Comparison of BER performance between [Qi et.al.] and our model for DF. The received $\mathrm{SNR}$ is $10 \mathrm{~dB}$. Source at $\mathrm{x}, \mathrm{y}=(0,0)$ and destination at $\mathrm{x}, \mathrm{y}=(1,0) .56$

4.19 Comparison of throughput performances of AF and DF using our power optimized model. The received $\mathrm{SNR}$ is $10 \mathrm{~dB}$. Source at $\mathrm{x}, \mathrm{y}=(-0.5,0)$ and destination at $\mathrm{x}, \mathrm{y}=(0.5,0) \ldots \ldots \ldots \ldots$. . . . . . . . . . 56

5.1 Receiver structure for M-FSK with noncoherent modulation. . . . . . . . . .

5.2 Comparison of simulated BER performance of AF with various different power allocations in Phase 1 and Phase 2 and direct communication. The envelope represents the best $\operatorname{BER}$ performance given by $\min _{p_{1}, p_{2}} \operatorname{BER}\left(p_{1}, p_{2}, d\right) . . .$.

5.3 Comparison of simulated BER performance of DF with various different power allocations in Phase 1 and Phase 2 and direct communication. The envelope represents the best $\operatorname{BER}$ performance given by $\min \operatorname{BER}\left(p_{1}, p_{2}, d\right)$. . . . . .

5.4 Comparison of simulated BER performance of AF and DF with various different power allocations in Phase 1 and Phase 2. The straight line represents direct communication. . . . . . . . . . . . . . . . . . .

5.5 Comparison of simulated BER performance of AF with various different power allocations in Phase 1 and Phase 2 and Qi's power allocation scheme. The envelope represents the best BER performance given by $\min _{p_{1}, p_{2}} \operatorname{BER}\left(p_{1}, p_{2}, d\right)$. .

5.6 Comparison of simulated BER performance of AF with various different power allocations in Phase 1 and Phase 2 and Qi's original and modified power allocation scheme. The envelope represents the best BER performance given by $\min _{p_{1}, p_{2}} \operatorname{BER}\left(p_{1}, p_{2}, d\right) \ldots \ldots \ldots \ldots \ldots$

5.7 Comparison of simulated BER performance of DF with various different power allocations in Phase 1 and Phase 2 and Qi's original and modified power allocation scheme. The envelope represents the best BER performance given by $\min _{p_{1}, p_{2}} \operatorname{BER}\left(p_{1}, p_{2}, d\right) \ldots \ldots \ldots \ldots \ldots$

5.8 Comparison of BER performances of AF and DF with power allocation modified from Qi's, and direct transmission for received SNRs of $10 \mathrm{~dB}$ and $20 \mathrm{~dB}$.

5.9 Comparison of throughput performances of AF and DF with power allocation modified from Qi's, and direct transmission for received SNRs of 10dB and $20 \mathrm{~dB} . \ldots \ldots \ldots \ldots \ldots \ldots \ldots$ 
5.10 BER performance of AF, with power allocation modified from Qi's, and direct transmission. The received SNR is $10 \mathrm{~dB}$. Source at $\mathrm{x}, \mathrm{y}=(-0.5,0)$ and destination at $\mathrm{x}, \mathrm{y}=(0.5,0) \ldots \ldots \ldots \ldots$

5.11 BER performance of DF, with power allocation modified from Qi's, and direct transmission. The received SNR is $10 \mathrm{~dB}$. Source at $\mathrm{x}, \mathrm{y}=(-0.5,0)$ and destination at $\mathrm{x}, \mathrm{y}=(0.5,0) \ldots \ldots \ldots \ldots$

5.12 BER performances of noncoherent DF, with power allocation modified from Qi's with received SNRs of $10 \mathrm{~dB}$ and $20 \mathrm{~dB}$. Source at $\mathrm{x}, \mathrm{y}=(-0.5,0)$ and destination at $\mathrm{x}, \mathrm{y}=(0.5,0) \ldots \ldots \ldots \ldots \ldots$

5.13 Throughput performance of noncoherent DF, with power allocation modified from Qi's with received SNRs of $10 \mathrm{~dB}$ and 20dB. Source at $\mathrm{x}, \mathrm{y}=(-0.5,0)$ and destination at $\mathrm{x}, \mathrm{y}=(0.5,0) \ldots \ldots \ldots \ldots$

5.14 The inner lighter area represents the region for relaying in AF with noncoherent modulation. The received $\mathrm{SNR}$ is $10 \mathrm{~dB}$. Source at $\mathrm{x}, \mathrm{y}=(-0.5,0)$ and destination at $\mathrm{x}, \mathrm{y}=(0.5,0) \ldots \ldots \ldots \ldots$

5.15 The inner lighter area represents the region for relaying in DF with noncoherent modulation. The received $\mathrm{SNR}$ is $10 \mathrm{~dB}$. Source at $\mathrm{x}, \mathrm{y}=(-0.5,0)$ and destination at $\mathrm{x}, \mathrm{y}=(0.5,0) \ldots \ldots \ldots \ldots$

5.16 The inner lighter areas represent the region for relaying in noncoherent environment AF. The received SNR for the left hand plot is $10 \mathrm{~dB}$ and that for the right hand one is $20 \mathrm{~dB}$. Source at $\mathrm{x}, \mathrm{y}=(-0.5,0)$ and destination at $\mathrm{x}, \mathrm{y}=$ $(0.5,0) \ldots \ldots \ldots \ldots \ldots \ldots$

5.17 The inner lighter areas represent the region for relaying in noncoherent environment using DF. The received SNR for the left hand plot is $10 \mathrm{~dB}$ and that for the right hand one is $20 \mathrm{~dB}$. Source at $\mathrm{x}, \mathrm{y}=(-0.5,0)$ and destination at $\mathrm{x}, \mathrm{y}$ $=(0.5,0) \ldots \ldots \ldots \ldots \ldots \ldots \ldots$

.1 The left hand plot shows the Gaussian probability density function with 0 mean and standard deviation 1 . The shaded area represents the area under the tail of a Gaussian random variable i.e. $\operatorname{Pr}\left(x \geq x_{0}\right)$. The right hand plot represents the plot of the Q-function. . . . . . . . . . . . 


\section{Notation}

We use the following notation and symbols throughout this thesis.

$Q(\cdot)$
$(\cdot)^{*}$
$\mathrm{E}[\cdot]$
$\|\cdot\|$
$d_{s, d}$
$d_{s, r}$
$d_{r, d}$
$r_{s, d}$
$r_{s, r}$
$r_{r, d}$
$n_{s, d}$
$n_{s, r}$
$n_{r, d}$
$k$
$\beta$
$\mathcal{P}_{\text {Total }}$
$p_{1}$
$p_{2}$
$\eta$
$\mathrm{AF}$
$\mathrm{DF}$
$\gamma_{\mathrm{AF}}$
$\gamma_{\mathrm{DF}}$
$\mathrm{BER} \mathrm{min}(d)$

: Q-function

: Complex conjugate

: Expectation operator

: Euclidian norm

: Source to destination distance

: Source to relay distance

: Relay to destination distance

: Signal received at destination from source

: Signal received at relay from source

: Signal received at destination from relay

: AWGN noise in the source to destination path

: AWGN noise in the source to relay path

: AWGN noise in the relay to destination path

scaling factor for received SNR

: $\left\|r_{s, r}\right\|^{-1}$

: Total transmit power in our system

: Phase 1 transmit power

: Phase 2 transmit power

: Bernoulli random variable

: Amplify and forward

Decode and forward

Instantaneous SNR of our system for coherent AF

Instantaneous SNR of our system for coherent DF

Best BER among a family of curves with different $p_{1}$ for a particular $\mathrm{d}$ and $p_{2}$ over a varying source to relay distance 


\section{Chapter 1}

\section{Introduction}

Starting from the days of smoke signals, signalling with mirrors, and semaphore codes, wireless communication has made blazing progress of late, a fact which is attested by the very common occurrence of lightweight, energy efficient mobile units capable of reliably handling voice and high-data-rate applications. The problem of fading and multipath, leading to degradation of system performance, was quickly recognized, and efforts mounted to alleviate these problems. The problems of multipath, like time delay and synchronization, loomed large, but the bane was soon turned into a boon with information harvesting from the independent signal paths to achieve performance benefits. This really started an era of cutting edge research aimed at improving the system performance. Research on different types of diversity, which exploits various kinds of signal or channel redundancy to improve performance, and development of multiple input multiple output (MIMO) systems, which have 2 or more transmit and receive antennas and use the multiple signal paths to robustly carry information across the channel $[1,2,3,4,5,6,7]$, and spacetime block codes (STBC) $[8,9,10,11]$, followed, offering performance improvement over single input single output systems.

The resulting evolution took wireless communication to a state where ad hoc wireless networks $[12,13,14,15,16,17,18]$ are no longer impossible to realize. Ad hoc networks are a group of mobile wireless nodes, with no centralized infrastructure, which can self-configure to form a network. In ad hoc networks, the network topology depends on the position and mobility of the nodes, so due to an arbitrary topology, such networks have to self configuring. 
The primary way they differ from infrastructure-based networks is that they have a fixed topology and require access points, base stations, and the connecting network to operate, whereas ad hoc networks can work without needing any of these.

Most modern wireless communications use frequencies in the gigahertz range, resulting in wavelengths which are less than a meter. MIMO systems require the presence of multiple antennas which can provide uncorrelated values of the signal at the receiver for combining, necessitating at least half a wavelength antenna spacing, which is not possible with the small mobiles currently in use. So not only for cellular systems but also ad hoc networks, which we consider in this thesis, and any other non-infrastructure based systems, where size limitations makes MIMO impractical, the idea of distributed or cooperative MIMO [19, 20, 21, 22] was introduced. In distributed MIMO the antennas of several single antenna devices cooperate to function on a whole as a MIMO system. With ad hoc networks, concepts like different forwarding techniques, optimal resource allocation, and cooperative relaying, came to the forefront.

In relay networks, the information transmitted from the transmitter is relayed by the nodes forming the network in order to improve the received signal. Unlike wireline multihop networking, where the relay nodes are interconnected by cables assuring a certain quality of service, the fading nature of the wireless channel, along with multipath interference, can cause considerable degradation of the transmitted signal. Due to the broadcast nature of radio all the relay nodes receive a copy of the transmitted signal but some of the copies might be highly degraded. Hence, the quality of the received signal might vary and this variation might not be insignificant, making routing more complicated than in wired networks.

Though in this thesis we do not assume cooperation between relays, we will briefly discuss the topic. A novel feature of cooperative relay networks is that the relays can cooperate with each other to extract the best possible output by using the relays in ways to optimize real system performance metrics like SNR, BER, achievable rates and throughputs. For example, the nodes communicate with each other to know which one is in the best position to relay or if a node does not have a good copy of the signal it looks for ones which might have a better copy. In the case of distributed or cooperative MIMO, the system shares the antennas of the participating nodes to from a virtual antenna array, thus mimicking a MIMO network. 
The independence of the various signal paths ensures that the probability of deep fade, which adversely affect signal quality, in all the different paths simultaneously is considerably lowered, resulting in overall performance improvement. But this improvement has to be balanced with the penalty of complicated routing and network control structure.

Research using an information theoretic approach to fully understand the working of ad hoc networks also ensued, and though great strides were made, a lot more remains to be understood. But results already obtained on performance metrics like system capacity and outage probability $[23,24,25]$ are acting as stepping stones for further research. Though information theoretic approaches offer a broader understanding of a system and give us a glimpse of the promise but it is ultimately performance metrics like end-to-end BER, the instantaneous SNR, their relationship, throughput, delay, achievable rates etc., that give us more details about practical performance. In this thesis we investigate end-to-end BER and throughput of a system in a relaying environment and tried to come up with improvements, using practical methods in a practical SNR regime.

The ways the nodes can convey or forward the signal has been well studied and developed, with techniques like, detect and forward, where the received signal is detected and then forwarded, decode and forward, where the received signal is decoded and then forwarded, and amplify forward, where the relay amplifies the received signal before forwarding, forming the base. These formed the nucleus around which more complex, exotic and practical forwarding techniques $[26,27,28,29,30]$ are developed. With extreme miniaturization of electronic components, the relay nodes have become smarter and can do much more than just "amplify and relay". They support various complicated forwarding techniques, requiring signal processing capabilities, routing algorithms, power control and other related functionalities. All these activities require power and for devices with finite power source, saving every drop of it becomes important. At the same time there is a need to maintain and improve overall system performance, thus emphasizing the development of schemes ensuring both quality of service and power savings.

In wireless communications the major problem of fading can be mitigated to a large extent by using large amounts of transmit power. But if we are trying to save power, this is not a practical solution. This necessitates optimal power allocation with a total 
system power constraint. Power control is important for infrastructure-based-networks, but it assumes much more importance in ad hoc networks, specially in sensor networks, where the relays consume very little power, in the range of $100 \mu \mathrm{W}$ [31]. But most of these relays are not rechargeable, making power optimization a big battery-life-enhancing and performanceimproving factor. Even when cell phones cooperate with each other, improving the overall system performance, they use precious battery power, thus making power optimization an important factor here too.

The effect of the distance between the wireless transmitter and receiver on system performance in various environments, has been studied extensively. It has been observed that there is loss of average signal power as the receiver moves farther away from the transmitter. This is caused by factors like shadowing and free space propagation from a point source. The receiver is also found to be sensitive to the instantaneous SNR of the received signal. There are a number of different models trying to capture this effect in various environments. In situations where intermediate relay nodes are used, if we can optimally allocate power, based on the position of the relay relative to the source or destination and instantaneous SNR, considerable overall performance improvement can be achieved.

Received signals can be demodulated in various ways depending on the availability of carrier phase and channel state information at the receiver, allowing for coherent demodulation when the information about the carrier phase and channel state are available. But in fading environments, extracting carrier phase becomes difficult, and it makes more practical sense to use noncoherent modulation where knowledge of carrier phase is not required. We consider both the coherent and noncoherent demodulation scenarios and worked on arriving at end-to-end performance boosting strategies in both the environments.

Another important issue is how to get a fairly accurate estimate of the relative distances among the nodes in the network. This is not much of a problem in networks with fixed topology but becomes critical in networks with dynamically changing topology. Nowadays it is common for networks to have bursty communications and shut down at times to save power. A portion of this latency can be used by the nodes to update their relative positions. The relay nodes can also update the transmitter about their positions using the uplink channel before the beginning of transmission. 
Now we will introduce the methodology followed in the thesis for improving system performance using position dependant power optimization in coherent and noncoherent environments employing amplify and forward and decode and forward techniques in relay networks. In the thesis we consider both coherent and noncoherent demodulation as well as forwarding techniques viz. amplify and forward and decode and forward and concentrate on allocating the optimal amount of power to the transmitter and the relay in these scenarios to improve system performance.

As discussed before, much of the current research has focussed on approaching the system from an information theoretic angle, optimizing various parameters like SNR and throughput based on capacity, outage probability, etc. [32, 33, 34, 35, 36, 37]. In contrast, here we develop a practical system, taking into account fading and path loss parameters, and we optimize power based on the instantaneous received SNR and the position of the relay to obtain overall BER and throughput enhancements. BER is important for low cost systems that cannot afford forward error correcting codes.

In Chapter 2 we present a general overview of concepts used in thesis. We discuss additive white Gaussian channels and move on to Rayleigh fading channels. Path loss models are then explained. A brief overview of relay networks is also provided. In Chapter 3 we introduce our system model and investigate the performance of amplify and forward with coherent demodulation. We propose a position dependant optimal power allocation strategy and identify the region where relaying is useful in this case. We also indicate the effects of different SNRs on the system performance, in general, and the region where relaying is useful, in particular. In Chapter 4, the same general strategy as Chapter 3 is followed, where decode and forward is used at the relay with coherent modulation, and performance of the power optimized protocol is compared with Qi et. al.'s work [37], which is based on information theoretic approaches. The region of interest, where relaying is useful, is also identified. We also compare the performance of amplify and forward with decode and forward. Chapter 5 deals with both of the forwarding protocols in a noncoherent enviornment. Using the power optimization used in [37] with some slight modifications, we investigate the BER and throughput performance and compare the results for both protocols. We also specify the area of relaying, which we define as the area where relaying provides better performance 
than direct communication, in all these cases. We draw our conclusions in Chapter 6 and review the salient results achieved and put forth some ideas about future work. 


\section{Chapter 2}

\section{Wireless Communication Systems and Relay Networks}

In this chapter, we review some of the basic concepts of wireless communications and relay networks that are used in the thesis. Since these topics are already well understood we choose to exercise brevity while describing them.

\subsection{Linear Modulation and Optimal Detection in AWGN channels}

We will develop a signal model at the input of the receiver. Let us assume that the transmitter uses a set of $M$ signal waveforms $\left\{s_{m}(t), m=1,2, \ldots, M\right\}$ to send information. These waveforms represent binary data, which may be a single bit or a few bits grouped together in a certain manner and are referred to as symbols. Here we consider signal transmission over the interval $0 \leq t \leq T$ where $T$ is the symbol or signalling interval. The channel introduces some noise and distortions in the transmitted signal. Quality of the signal may degrade due to distortion caused by factors like fading, multipath, intersymbol interference and interference due to multiuser and narrowband noise, but the additive portion of noise is modeled as a white complex Gaussian process and is present in all frequencies of interest. This is termed as additive white Gaussian noise (AWGN). The received signal under AWGN 
channel in the interval $0 \leq t \leq T$, is given by

$$
r(t)=s_{m}(t)+n(t), \quad m=1,2, \ldots, M .
$$

Samples of $n(t)$, the continuous time white Gaussian noise process, are independent with 0 mean and power spectral density of $\frac{N_{0}}{2}$ per dimension.

We should design a receiver which should be able to estimate the transmitted signal based upon the observation of $r(t)$. Here, we explain the design of an optimum receiver that minimizes the probability of error, while estimating the received signal. The receiver broadly consists of two blocks, the demodulator and the detector. The function of the demodulator is to convert the continuous time received signal into discrete time signal vector, producing sufficient statistics for detection and the detector tries to arrive at a good estimate of the transmitted signal. The demodulator can be implemented in the form of a correlation demodulator or a matched filter demodulator either of these devices can produce sufficient statistics. The matched filter can be used to maximize the output SNR but here we consider the correlation demodulator.

\section{The Correlation Demodulator}

We expand the signal and noise into a series of $K$ orthonormal basis functions $\left\{f_{k}(t)\right\}, k=$ $1,2, \ldots, K$, spanning the signal space, without discarding any useful information. The correlation demodulator decomposes the received signal and noise into $K$-dimensional vectors which form the sufficient statistic. In Fig. 2.1, a correlation-type demodulator is shown, where the received signal $r(t)$ is first passed through a bank of $K$ parallel correlators, which compute the projection of $r(t)$ onto the $K$ orthonormal basis functions resulting in the vector

$$
\boldsymbol{r}=\left[r_{1}, r_{2}, \ldots, r_{K}\right]
$$

So the demodulator output can be written as

$$
r_{k}=s_{m k}+n_{k}, \quad k=1,2, \ldots, K
$$

where

$$
r_{k}=\int_{0}^{T} r(t) f_{k}(t) d t
$$




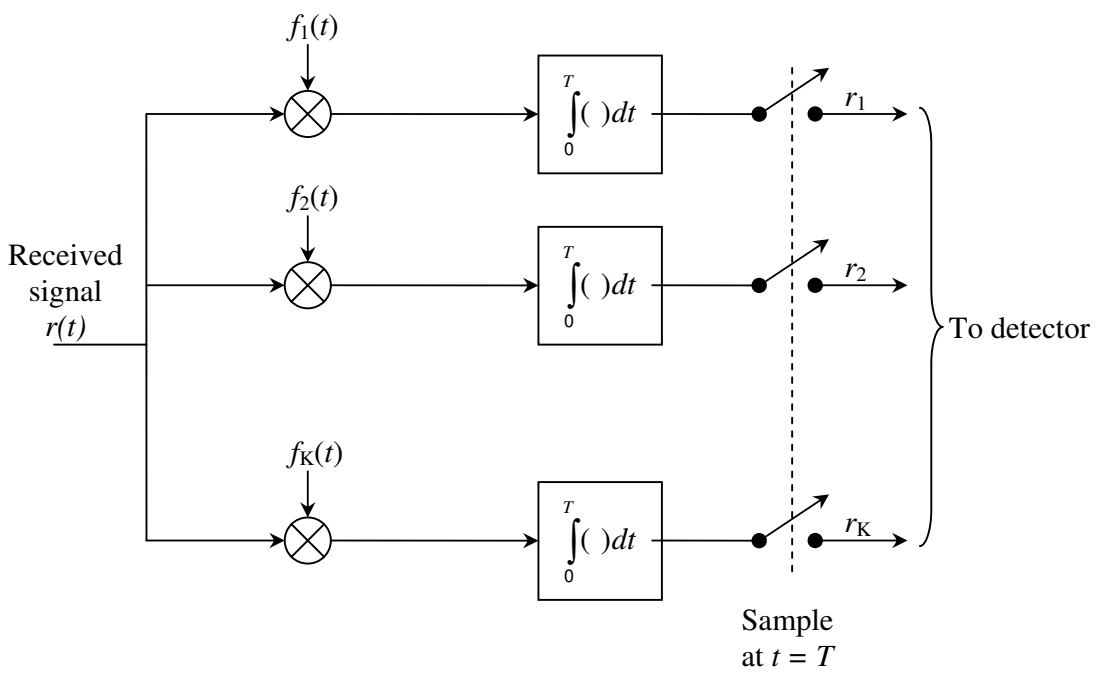

Figure 2.1: correlation-type demodulator.

$$
\begin{gathered}
s_{m k}=\int_{0}^{T} s_{m}(t) f_{k}(t) d t \\
n_{k}=\int_{0}^{T} n(t) f_{k}(t) d t .
\end{gathered}
$$

Now the signal can be represented by using the vector $\boldsymbol{s}_{m}=\left[s_{m 1}, s_{m 2}, \ldots, s_{m K}\right]$. We note here that the basis functions $\left\{f_{k}(t)\right\}$ do not span the noise space. The noise $n(t)$ can be represented as

$$
n(t)=n^{\prime}(t)+\sum_{i=1}^{K} n_{k} f_{k}(t) .
$$

Then, the uncorrealted portion of the noise

$$
n^{\prime}(t)=n(t)-\sum_{k=1}^{K} n_{k} f_{k}(t)
$$

represents the difference between the original noise process and the projection of $n(t)$ onto the orthonormal basis functions $f_{k}(t)$. The received bandpass signal in the interval $0 \leq t \leq T$ can be expressed as

$$
r(t)=\sum_{k=1}^{K} r_{k} f_{k}(t)+n^{\prime}(t) .
$$


It can be shown that the uncorrelated or orthogonal noise, $n^{\prime}(t)$ can be disregarded by the receiver since it is does not affect the decision on the transmitted signal [38]. The $r_{k}$ 's are also known as sufficient statistics as they contain sufficient information to make a decision at the detector, on which of the $M$ signals was transmitted. We can thus represent the received signal also as

$$
\boldsymbol{r}=\boldsymbol{s}_{m}+\boldsymbol{n}
$$

where $\boldsymbol{n}=\left[n_{1}, n_{2}, \ldots, n_{K}\right]$. This is known as the signal space approach.

\section{The Optimum Detector}

Using the signal space approach, we have seen that a vector $\boldsymbol{s}_{m}=\left[s_{m 1}, s_{m 2}, \ldots, s_{m K}\right]$ can be transmitted and a vector

$$
\boldsymbol{r}=\left[r_{1}, r_{2}, \ldots, r_{K}\right]=\boldsymbol{s}_{m}+\boldsymbol{n}
$$

is received, where individual elements of the vector $\boldsymbol{n}$ are i.i.d (independent and identically distributed) Gaussian random variables. Given $\boldsymbol{r}$, the detector has to form an estimate $\hat{\boldsymbol{s}}_{m}$ of the transmitted signal vector which minimizes the probability of symbol error. The optimum detector detector would be one which implements the maximum a posteriori probability (MAP) decision rule which minimizes the symbol error probability by choosing the signal $\boldsymbol{s}_{m}$ satisfying

$$
\operatorname{Pr}\left(\boldsymbol{s}_{m} \mid \boldsymbol{r}\right) \geq \operatorname{Pr}\left(\boldsymbol{s}_{i} \mid \boldsymbol{r}\right), \quad \forall m \neq i
$$

Or equivalently,

$$
\frac{p\left(\boldsymbol{r} \mid \boldsymbol{s}_{m}\right) \operatorname{Pr}\left(\boldsymbol{s}_{m}\right)}{p(\boldsymbol{r})} \geq \frac{p\left(\boldsymbol{r} \mid \boldsymbol{s}_{i}\right) \operatorname{Pr}\left(\boldsymbol{s}_{i}\right)}{p(\boldsymbol{r})}, \quad \forall m \neq i .
$$

Here $p(\boldsymbol{r})$ is the pdf of the received vector and $p\left(\boldsymbol{r} \mid \boldsymbol{s}_{m}\right)$ is the conditional pdf of the observed vector $\boldsymbol{r}$ given $\boldsymbol{s}_{m}$. If the a priori probabilities $\operatorname{Pr}\left(\boldsymbol{s}_{1}\right)=\ldots=\operatorname{Pr}\left(\boldsymbol{s}_{M}\right)$ are equal, then the MAP rule simplifies to maximum likelihood (ML) decision rule. For the AWGN channel, ML decision rule finds the signal $\boldsymbol{s}_{m}$ that is closest in Euclidian distance sense given by, 
$\boldsymbol{D}\left(\boldsymbol{r}, \boldsymbol{s}_{m}\right)=\sum_{k=1}^{K}\left(r_{k}-s_{m k}\right)^{2}$, to the received signal vector $\boldsymbol{r}$. Thus the ML decision rule can be summarized as

$$
p\left(\boldsymbol{r} \mid \boldsymbol{s}_{m}\right) \geq p\left(\boldsymbol{r} \mid \boldsymbol{s}_{i}\right), \quad \forall m \neq i
$$

\subsection{BPSK in Additive White Gaussian Noise Channel}

The simplest form of phase shift keying modulation is binary phase shift keying (BPSK) modulation, which has a signal space dimension of $K=1 . E_{b}$ is the signal energy per bit and the basis function $f_{1}(t)$, defined in the interval $0 \leq t \leq T$, is given by

$$
f_{1}(t)=\sqrt{\frac{2}{T}} \cos \left(2 \pi f_{c} t\right),
$$

where $f_{c}$ is the carrier frequency. Here one of the two equiprobable signals given by

$$
s_{1}(t)=\sqrt{E_{b}} f_{1}(t), \quad s_{2}(t)=-\sqrt{E_{b}} f_{1}(t), \quad 0 \leq t \leq T
$$

is transmitted. These signals can be represented in a one dimensional signal space, spanned by $f_{1}(t)$, shown by Fig. 2.2 , as real numbers by

$$
s_{1}=\sqrt{E_{b}}, \quad s_{2}=-\sqrt{E_{b}} .
$$

The received signal in this case is given by (2.10).

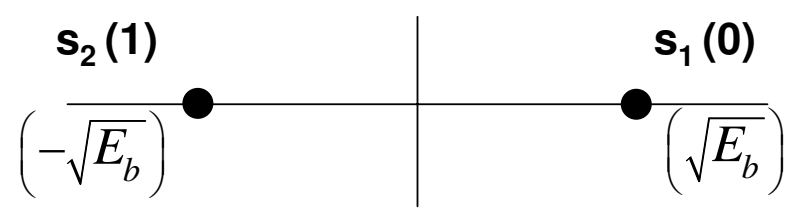

Figure 2.2: BPSK constellation .

The probability of symbol error and probability of bit error for BPSK in AWGN channel is same and is given by,

$$
P_{s}=P_{b}=Q\left(\sqrt{\frac{2 E_{b}}{N_{0}}}\right)
$$


where, $\frac{E_{b}}{N_{0}}$ is the signal to noise ratio (SNR) at the receiver. For BPSK we can use $E_{s}$, the energy per symbol and $E_{b}$, the energy per bit, interchangeably as symbols and bits are the same for BPSK.

\subsection{QPSK in Additive White Gaussian Noise Channel}

We consider the quadrature phase shift keying (QPSK) signal set, having a signal space dimension of 2 , as

$$
\begin{aligned}
& s_{1}(t)=\sqrt{\frac{E_{s}}{2}} f_{1}(t)+\sqrt{\frac{E_{s}}{2}} f_{2}(t) \\
& s_{2}(t)=-\sqrt{\frac{E_{s}}{2}} f_{1}(t)+\sqrt{\frac{E_{s}}{2}} f_{2}(t) \\
& s_{3}(t)=-\sqrt{\frac{E_{s}}{2}} f_{1}(t)-\sqrt{\frac{E_{s}}{2}} f_{2}(t) \\
& s_{4}(t)=\sqrt{\frac{E_{s}}{2}} f_{1}(t)-\sqrt{\frac{E_{s}}{2}} f_{2}(t) .
\end{aligned}
$$

These transmitted signals are represented using the basis functions

$$
\begin{aligned}
& f_{1}(t)=\sqrt{\frac{2}{T}} \cos \left(2 \pi f_{c} t\right) \\
& f_{2}(t)=\sqrt{\frac{2}{T}} \sin \left(2 \pi f_{c} t\right) .
\end{aligned}
$$

The constellation diagram for (QPSK) using Gray coding [39] is shown in Fig. 2.3. Using the 2-dimensional signal space the symbols can be represented as $\boldsymbol{s}_{1}=\left[\sqrt{\frac{E_{s}}{2}}, \sqrt{\frac{E_{s}}{2}}\right]^{T}, \boldsymbol{s}_{2}=$ $\left[-\sqrt{\frac{E_{s}}{2}},{\sqrt{\frac{E_{s}}{2}}}^{T}, \boldsymbol{s}_{3}=\left[-\sqrt{\frac{E_{s}}{2}},-{\sqrt{\frac{E_{s}}{2}}}^{T}, \boldsymbol{s}_{4}=\left[\sqrt{\frac{E_{s}}{2}},-{\sqrt{\frac{E_{s}}{2}}}^{T}\right.\right.\right.$. The reason for using Gray coding is that there is a difference of only a bit between adjacent symbols, ensuring that generally a single bit error will not change the output to nonadjacent symbols. For example in Fig. 2.3 symbol $s_{2}(01)$ can change to $s_{1}(00)$ or $s_{3}(11)$, with a single bit error but there has to be two bits in error to change it to $s_{4}(10)$.

The received signal in this case is given by (2.10). The symbol error probability for QPSK in AWGN channel can be given as

$$
\begin{aligned}
P_{s} & =2 Q\left(\sqrt{\frac{2 E_{b}}{N_{0}}}\right)-\left[Q\left(\sqrt{\frac{2 E_{b}}{N_{0}}}\right)\right]^{2} \\
& \left.\approx 2 Q\left(\sqrt{\frac{2 E_{b}}{N_{0}}}\right) \text { (Since } Q^{2}(.)<<Q(.)\right) .
\end{aligned}
$$




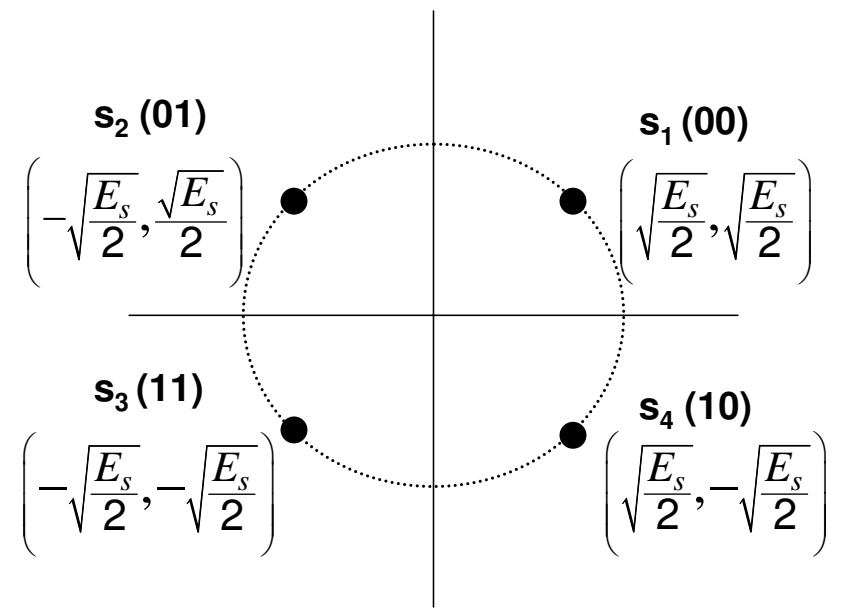

Figure 2.3: QPSK constellation diagram with Gray coding.

For Gray coding and also noting that $\gamma_{s}=2 \gamma_{b}$, where $\gamma_{s}$ is the SNR per symbol and $\gamma_{b}$ is the SNR per bit, we can write the bit error probability for QPSK in AWGN as,

$$
P_{b}=Q\left(\sqrt{2 \gamma_{b}}\right)
$$

QPSK can be thought as two orthogonal binary phase shift keying (BPSK) constellations and the bit error probability for QPSK is the same as BPSK.

\subsection{Coherent PSK in Rayleigh Fading Channel}

First we will briefly describe the expressions needed to calculate error probability in Rayleigh fading.

\section{Error Probability in Rayleigh Fading Channel}

We can express the average probability of bit error, $P_{b}$, in a frequency-nonselective, slow, Rayleigh block fading channel as [40]

$$
P_{b}=\int_{0}^{\infty} P_{b}\left(\gamma_{b}\right) p\left(\gamma_{b}\right) d \gamma_{b}
$$

where we define, $P_{b}\left(\gamma_{b}\right)$ as the probability of error for any modulation conditioned on the bit SNR $\gamma_{b}$ and $p\left(\gamma_{b}\right)$ is the probability density function of $\gamma_{b}$. Also $\gamma_{b}$ has a chi-square 
distribution with two degrees of freedom. We define $\Gamma \triangleq \mathrm{E}\left[\gamma_{b}\right]$, as the average value of SNR in fading environment and then $p\left(\gamma_{b}\right)$ can be expressed as,

$$
p\left(\gamma_{b}\right)=\frac{1}{\Gamma} \exp \left(-\frac{\gamma_{b}}{\Gamma}\right), \gamma_{b} \geq 0
$$

We can now use (2.29) and the probability of error of BPSK in AWGN in (2.28) to get the bit error probability of BPSK in Rayleigh fading as

$$
P_{b}=\frac{1}{2}\left[1-\sqrt{\frac{\Gamma}{1+\Gamma}}\right] .
$$

Using high SNR approximation, (2.30) can be written as

$$
P_{b} \approx \frac{1}{4 \Gamma}
$$

The bit error probability of QPSK in AWGN is same as that of BPSK, so they have the same probability of bit error in Rayleigh fading too, given by (2.30).

\subsection{FSK in Rayleigh Fading Channel}

Frequency-shift keying (FSK) is a form of frequency modulation in which the modulating signal shifts the output frequency between predetermined values. In $M$-ary FSK modulation the $M$ transmitted signals are generally of equal energy and duration. Each of the $M$ symbols are represented by a particular frequency which are orthogonal to each other. It can be continuous in phase or discontinuous in phase depending on the generation method. FSK signal can be detected coherently or noncoherently. In this section we will discuss coherent detection of FSK. For 4-FSK, which has a signal space dimension of 4, the signals can be represented as

$$
\begin{aligned}
& s_{1}(t)=\sqrt{E_{s}} f_{1}(t) \\
& s_{2}(t)=\sqrt{E_{s}} f_{2}(t) \\
& s_{3}(t)=\sqrt{E_{s}} f_{3}(t) \\
& s_{4}(t)=\sqrt{E_{s}} f_{4}(t)
\end{aligned}
$$


where the basis functions can be represented as

$$
f_{i}(t)=\sqrt{\frac{2}{T}} \cos \left(2 \pi f_{i} t\right) \text { for } i=1,2, \ldots, 4 .
$$

We can write

$$
f_{i}=f_{c}+(i-1) \triangle f \text { for } i=1,2, \ldots, 4
$$

where $f_{c}$ is the carrier frequency and $\triangle f=\frac{1}{2 T}$ is the minimum separation between adjacent frequencies to maintain orthogonality. Using the signal space model the FSK symbols can be represented as $\boldsymbol{s}_{1}=[0,0,0,1]^{T}, \boldsymbol{s}_{2}=[0,0,1,0]^{T}, \boldsymbol{s}_{3}=[0,1,0,0]^{T}, \boldsymbol{s}_{4}=[1,0,0,0]^{T}$ in the case of 4-FSK. The optimum receiver for coherent $M$-ary FSK, as shown in Fig. 2.4, consists of a bank of $M$ correlators or matched filters tuned to the $M$ distinct carrier frequencies.

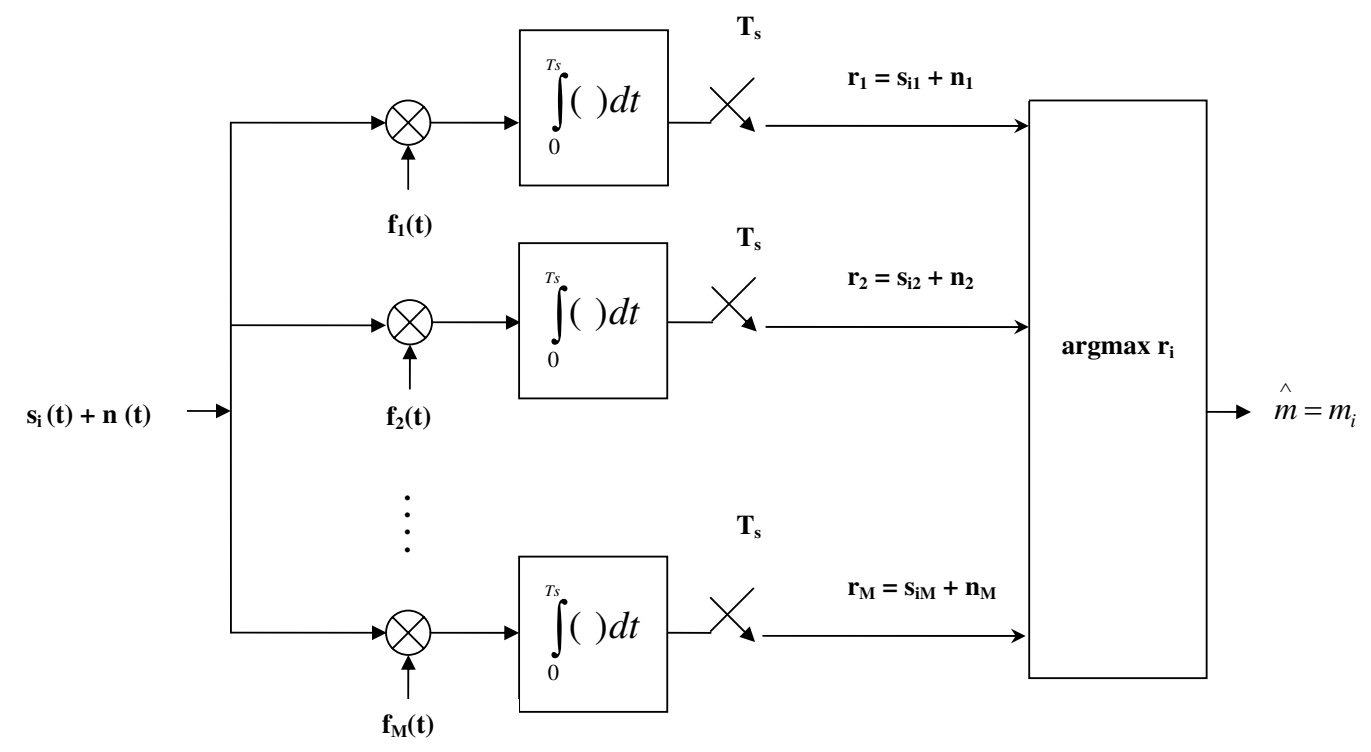

Figure 2.4: Receiver structure for M-FSK with coherent modulation.

Using the union bound, the average symbol error probability can bounded by [41]

$$
P_{s} \leq(M-1) Q\left(\sqrt{\frac{\log _{2}(M) E_{b}}{N_{0}}}\right) .
$$

The average bit error probability is given by 1

$$
P_{b}=\frac{M}{2(M-1)} P_{s} .
$$

\footnotetext{
${ }^{1}$ Derived in next section
} 
Now, by using (2.29) and substituting $P_{b}$ in (2.28), we can get the bit error probability for coherent FSK in Rayleigh fading. The performance of coherent FSK is seen to be better than noncoherent FSK but in the later case, carrier phase knowledge is not required.

\subsection{Noncoherent FSK in Rayleigh Block Fading Chan- nel}

For noncoherent 4-FSK modulation, having a signal space dimension of 4 , the signals are the same as coherent 4-FSK. Only the minimum separation between frequencies to maintain orthogonality is $\triangle f=\frac{1}{T}$. In the case of noncoherent $M$-FSK the carrier phase is not known and the received signal can be represented as

$$
r(t)=\sqrt{\frac{2 E_{s}}{T}} \cos \left(2 \pi f_{m}+\phi_{m}\right)+n(t) \quad \text { for } m=1,2, \ldots, 4
$$

where $f_{m}$ is the carrier frequency and $\phi_{m}$ is the phase of the carrier $f_{m}$. The demodulation of the received signal $r(t)$ can be achieved by using two correlators per carrier frequency. The basis functions corresponding to each carrier frequency are represented as

$$
\begin{aligned}
& f_{c m}(t)=\sqrt{\frac{2}{T}} \cos \left(2 \pi f_{m} t\right) \\
& f_{s m}(t)=-\sqrt{\frac{2}{T}} \sin \left(2 \pi f_{m} t\right) \text { for } m=1,2, \ldots, 4
\end{aligned}
$$

where

$$
f_{m}=f_{c}+(m-1) \triangle f \text { for } m=1,2, \ldots, 4
$$

with $f_{c}$ as the carrier frequency and $\triangle f=\frac{1}{T}$ is the minimum separation between adjacent frequencies to maintain orthogonality. We will now evaluate the probability of error of 4FSK with noncoherent detection in Rayleigh fading. The exact probability of symbol error of 4-FSK with noncoherent detection in AWGN can be written as [38],

$$
P_{s}=\sum_{n=1}^{M-1}(-1)^{n+1}\left(\begin{array}{c}
M-1 \\
n
\end{array}\right) \frac{1}{n+1} \exp \left[-\frac{n k \gamma_{b}}{n+1}\right]
$$


where $\gamma_{b}$ is the SNR per bit and $M=4$ and $k=\log _{2} M=2$ for our case. We assume equiprobable symbols so the bit error probabilities would also be equal and can be represented as

$$
\frac{P_{s}}{M-1}=\frac{P_{s}}{2^{k}-1}
$$

Also $n$ bits, $(n \leq k)$, out of $k$ bits could be in error, so the average number of bit errors for a $k$-bit symbol is

$$
\sum_{n=1}^{k} n\left(\begin{array}{l}
k \\
n
\end{array}\right) \frac{P_{s}}{2^{k}-1}=k \frac{2^{k-1}}{2^{k}-1} P_{s} .
$$

Then, we can calculate the bit error probability by dividing (2.46) by $k$, resulting in [38],

$$
P_{b}=\frac{2^{k-1}}{2^{k}-1} P_{s}
$$

Now by using (2.29) and substituting $P_{b}$ in $(2.28)$ we can get the bit error probability for noncoherent 4-FSK in Rayleigh fading.

\subsection{Diversity and Maximal Ratio Combining}

In order to combat fading and multipath, a range of techniques were developed that exploited some kind of channel or signal redundancy to improve system performance. These are collectively called diversity techniques. We can use diversity in a fading environment. It has been established [38] that if a channel is in deep fade, the signal suffers a considerable loss of quality. If we have multiple signal paths and the probability of deep fade in each is $p$, then with $L$ independent paths that would go down drastically to $p^{L}$, thereby increasing the chance of recovering the signal manifold. There are various kinds of diversity, like time diversity, frequency diversity and spatial diversity which are generally used to improve performance. Here we would discuss SNR improvement with Maximal Ratio Combining (MRC) [16, 42, 40].

After sampling the output of the matched filter, the received signal for branch $k(1 \leq$ $k \leq L)$ is

$$
\boldsymbol{r}_{k}=h_{k} \boldsymbol{s}_{m}+\boldsymbol{n}_{k}
$$


where $\boldsymbol{r}$ in $\boldsymbol{r}_{k}$ is defined in (2.11) and the subscript $k$ refers to each branch. The branches come from temporal, frequency, or time based diversity, and each branch is an independently faded copy of the signal. So for the $k^{\text {th }}$ branch the instantaneous SNR can be expressed as

$$
\gamma_{k}=\frac{E_{s}\left|h_{k}\right|^{2}}{N_{0}} .
$$

We assume symbols with equal energy given by $E_{s}$ and define the expectation operator as $\mathrm{E}[\cdot]$. Now if we assume that each branch has the same average SNR,

$$
\Gamma \triangleq \frac{E_{s}}{N_{0}} \mathrm{E}\left[\left|h_{k}\right|^{2}\right]
$$

then for Rayleigh fading, each branch can be said to have the same exponential SNR distribution, given by

$$
p(\gamma)=\frac{1}{\Gamma} e^{\frac{-\gamma}{\Gamma}}
$$

The SNR improvement in MRC comes from coherently combining (co-phasing) each diversity branch to provide the largest possible SNR. Supposing there are $L$ diversity branches available, the decision statistic can be written as

$$
\begin{aligned}
d & =\sum_{k=1}^{L}\left(h_{k}\right)^{*} \boldsymbol{r}_{k} \\
& =\sum_{k=1}^{L}\left|h_{k}\right|^{2} \boldsymbol{s}_{m}+\sum_{k=1}^{L}\left(h_{k}\right)^{*} \boldsymbol{n}_{k},
\end{aligned}
$$

yielding an instantaneous SNR of

$$
\begin{aligned}
\gamma_{\mathrm{MRC}} & =\frac{E_{s}\left(\sum_{k=1}^{L}\left|h_{k}\right|^{2}\right)^{2}}{N_{0} \sum_{k=1}^{L}\left|h_{k}\right|^{2}} \\
& =\frac{E_{s} \sum_{k=1}^{L}\left|h_{k}\right|^{2}}{N_{0}}
\end{aligned}
$$

It is seen that $\gamma_{\mathrm{MRC}}$ has a chi-square distribution with $2 L$ degrees of freedom, given by

$$
p_{L}(\gamma)=\frac{1}{(L-1) ! \Gamma^{L}} \gamma^{L-1} e^{\frac{-\gamma}{\Gamma}}
$$

and we can express the average SNR as

$$
\Gamma_{\mathrm{MRC}}=\mathrm{E}\left[\gamma_{\mathrm{MRC}}\right]=\Gamma L
$$


So it turns out that in MRC, SNR is harvested from all the branches including the low SNR ones. Thus all available energy from all diversity branches are coherently added in MRC, helping it to attain the largest possible average SNR. It is also seen that for MRC there is a change in the shape of the distribution too, which with increasing number of diversity branches, allows progressively fewer low SNR realizations, which in turn increases the slope of the SNR-BER curve. We can find the probability of error for BPSK in a $L$-branch diversity channel with MRC by averaging the BER of BPSK in AWGN, given ${ }^{2}$ by $Q(\sqrt{2 \gamma})$ over the distribution of the SNR (2.56). This results in [40]

$$
P_{b}=\left[\frac{1}{2}(1-\mu)\right]_{k=0}^{L} \sum_{k}^{L-1}\left(\begin{array}{c}
L-1-k \\
k
\end{array}\right)\left[\frac{1}{2}(1+\mu)\right]^{k}
$$

where,

$$
\mu \triangleq \sqrt{\frac{\Gamma}{1+\Gamma}}
$$

We can use the high-SNR approximation when $[42,40] \bar{\gamma} \geq 10 \mathrm{~dB}$,

$$
P_{b} \approx\left(\frac{1}{4 \Gamma}\right)^{L}\left(\begin{array}{c}
2 L-1 \\
L
\end{array}\right)
$$

So from Fig. 2.5 it is seen that the BER varies as $\frac{1}{\Gamma}$ raised to the $L^{\text {th }}$ power. Following a similar procedure, it can be shown that for coherent FSK at high SNR, the BER is

$$
P_{b} \approx\left(\frac{1}{2 \Gamma}\right)^{L}\left(\begin{array}{c}
2 L-1 \\
L
\end{array}\right)
$$

The value of $L$ represents the slope of the BER-SNR curve in the log domain and the equation,

$$
P_{b} \propto\left(\frac{1}{\Gamma}\right)^{L}
$$

is true for MRC for almost all modulation schemes. $L$ is called the diversity gain of the system and a system whose BER curve has the same slope as MRC with $L$-branches is said to exploit full $L$-branch diversity.

Fig. 2.5 indicates the change in BER with changing SNR, for a varied number of diversity branches. The diversity gain can clearly be seen from the increasing slope of BER curve,

\footnotetext{
${ }^{2}$ In this case $\gamma=\gamma_{b}$, the SNR per bit
} 


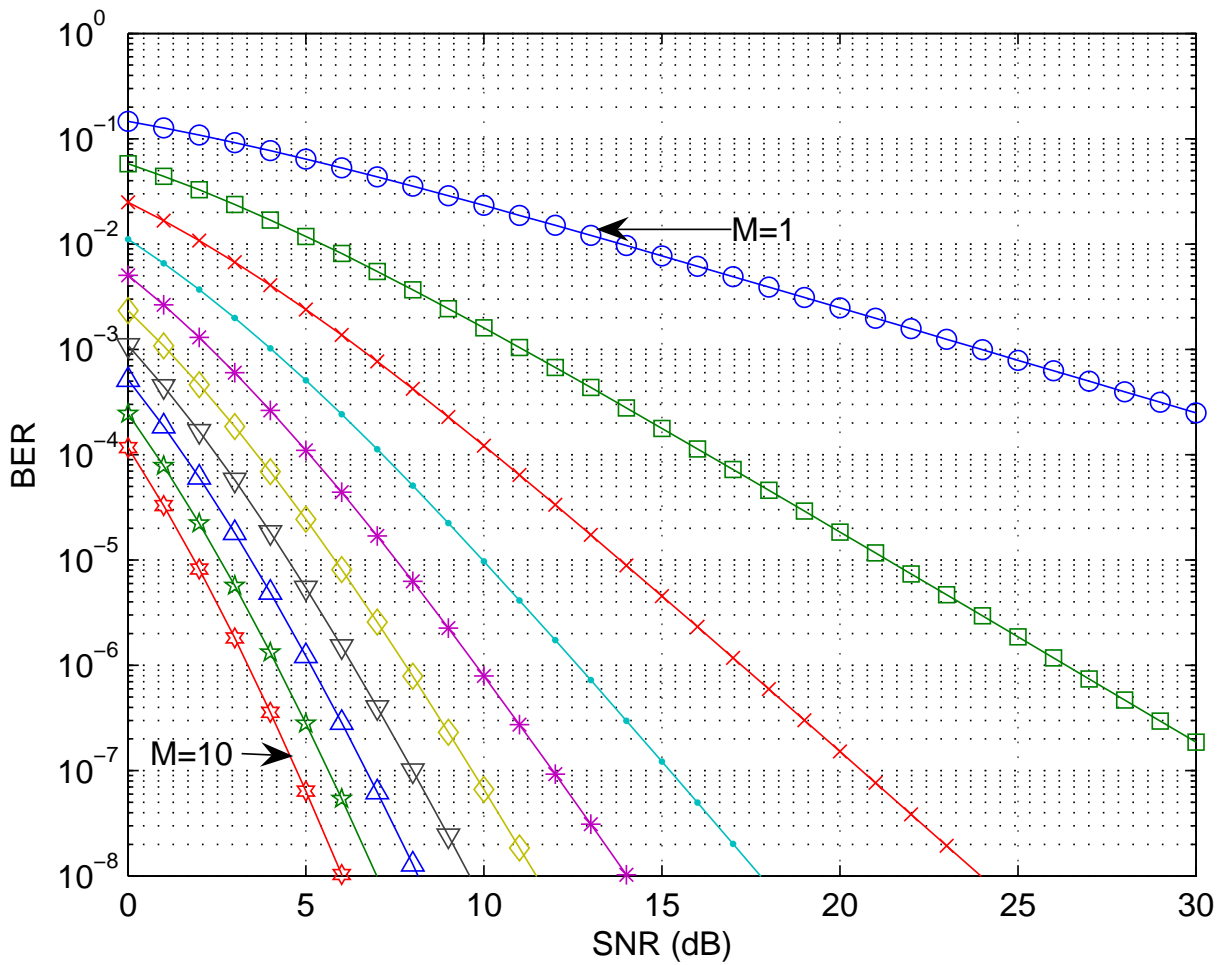

Figure 2.5: BER curve with maximum ratio combining for BPSK modulation having varied number of diversity branches.

which increases with the number of diversity branches. We have seen in [40] that in the $\log$ domain there is a linear relationship between BER and SNR in a Rayleigh fading channel but there is a distinct performance improvement, as shown by

$$
P_{b} \propto \frac{1}{\mathrm{SNR}^{L}}
$$

achieved by using diversity techniques. Lower BERs can be achieved at lower SNRs while using diversity techniques and that eventually translates to considerable power savings too.

\subsection{Path Loss Models}

\section{Path Loss and Large Scale Fading}

It has been observed that as the distance between transmitter and receiver increases there is a gradual decrease in the average received power. The gradual large-scale variation in the 
signal strength, which is simply a function of transmitter-receiver separation, is known as large scale fading. We would like to include the effect of distance between the transmitter and receiver in our system model which is discussed in detail in Chapter 3. We will briefly discuss two main path loss models.

\section{Log-Distance Path Loss Model}

Both theoretical and experimental propagation models showed that the mean received signal strength decreases logarithmically with distance for indoor and outdoor wireless channels [40, 31]. When free space path loss is considered, it is seen to be proportional to the square of the distance. But if perfect ground reflection is taken into account, the average received power falls off according to the fourth power of distance. Practical average large-scale path loss is given by,

$$
\overline{P L}(d)=\overline{P L}\left(d_{0}\right)+10 \alpha \log \left(\frac{d}{d_{0}}\right) \mathrm{dB}
$$

where $d$ is the distance between the transmitter and receiver, $d_{0}$ is the reference distance in the far field of the antenna, $\alpha$ is the path loss exponent whose value depends on the antenna height, frequency and the propagation environment. Measurements show that $\alpha$ varies between 2 in free space to 10 in dense urban environment. The value of path loss at the reference distance $d_{0}$ is usually found by using actual field measurements.

\section{Log-normal Shadowing}

The fact that the average path loss might be different, due to the difference of environmental clutter, for the same transmitter-receiver separation, has been established experimentally. This resulted in the Log-Normal Shadowing model [40, 31]. Experiments showed that the path loss at a particular distance is random and is log-normally distributed about its mean distance dependent value, $\overline{P L}(d)[43,44]$. So we can express this model as,

$$
\overline{P L}(d)=\overline{P L}\left(d_{0}\right)+10 \alpha \log \left(\frac{d}{d_{0}}\right)+X_{\sigma} \mathrm{dB}
$$

where where $X_{\sigma}$ is a normal random variable in $d B$, with standard deviation of $\sigma d B$, i.e. $X_{\sigma} \sim \mathcal{N}\left(0, \sigma^{2}\right)$. From (2.65) it is seen that the log-normal distribution describes the random shadowing effects occurring over a large number of measurement locations with same 
transmitter-receiver separation, but having different amounts of environmental clutter. Sending more signal power or a 'fade margin', which is the amount of excess power needed to overcome shadowing, helps to mitigate this problem.

\subsection{Relay Networks: An Overview}

The concept of relaying information was there even during historic times in a rudimentary form, but with the advent of radio it took a completely different meaning. Pioneering works by Claude Shannon [45] and Fano [46] opened up the potentials for communication in a much broader sense by laying the base for information theory. One of the earliest works on three terminal communication, where all three terminals cooperate to maximize the end to end capacity, was investigated by Edward van der Meulen in his 1970 paper, "Three-terminal communication channels" [47]. The coming years saw a deluge of information theoretic researches on multiterminal cooperative networks by Cover, Gamal, Aref etc. [48, 49, 50, 51, 52]. These seminal researches investigated topics like capacity of relay channels, broadcast channels, capacity of semi-deterministic relay channels, thus strengthening the foundation for further advances.

The field of relaying gradually evolved with concepts of different kinds of forwarding techniques like, observe and forward where the relay observes and forwards the signal, estimate and forward (Cover and El Gamal, 1979), wherein the relay estimates the received signal and then forwards. In quantize and forward the received signal is quantized by the relay before forwarding whereas in compress and forward (Kramer, Gastpar, Gupta, 2005) the received signal is compressed before forwarding. The relay amplifies the received signal before forwarding in amplify and forward and in decode and forward (Kramer, Gastpar, Gupta, 2005) (Laneman, 2002) the received signal is decoded and then forwarded [53]. More recently schemes like decode-amplify and forward, which combines decode and forward and amplify and forward has been proposed by X. Bao, and J. Li in [29].

The importance of resource allocation for relay networks was quickly realized and a lot of research on that topic followed. Reznik, Kulkarni and Verdu [35] worked on optimal power allocation and capacity of degraded Gaussian multirelay channel. Liang and Veeravalli 
worked on determining the optimal resource allocation and capacity for orthogonal Gaussian relay channels in [33]. Hasna and Alouini investigated the optimum power allocation for selective transmit-diversity systems over Nakagami fading channels [34]. The case of a large scale cooperative relaying network, with optimal coherent combining under aggregate relay power constraint, was dealt by Larsson [36], where he used a group of $48 \times 48$ relays in a square grid for evaluating cooperative relaying under power constraint. Maric and Yates showed in [54] that in a relay network, data should be transmitted over a single relay path which is in the best position in the network. In [55] a power allocation scheme was proposed for the more complex adaptive decode and forward protocol, where the destination has to know when the relay is forwarding, the source must have knowledge on the decoding status of the relay and there may also be requests for retransmission with the source repeating the message. Another power allocation scheme is proposed in [37], where the approach is from an information theoretic point of view with the aim of maximizing SNR and hence channel capacity, for a regenerative relay system, in Rayleigh fading environment, with instantaneous and mixed CSI at the transmitter. Most of the above works have assumed that the nodes are subject to sum power constraint.

In our thesis we will assume the same, sum power constraint and aim towards achieving better system performance in coherent and noncoherent modulation scenarios while using amplify and forward and decode and forward with position dependant optimal power allocation. 


\section{Chapter 3}

\section{Amplify and Forward with Coherent Modulation}

\subsection{System Model}

We have seen in the last chapter that there is a general performance gain offered by MRC. This gain sometimes offers a lot of overall performance boost in fading multipath environments. Taking motivation from this, we look at cooperative relaying for diversity gains. Of the various forwarding schemes used in the cooperative relay networks, amplify and forward is the probably the simplest one in terms of implementation and this has led to a lot of analysis of its various performance metrics. Before evaluating the performance of AF we would now proceed to describe our system model that we would be using throughout the thesis irrespective of the forwarding or modulation technique.

To simplify and facilitate the problem of power allocation, a single relay and half-duplex system model is used. Our system is a simple three terminal model as shown in Fig.3.1 with one source, one destination and one relay denoted by $s, d$ and $r$ respectively. The system is half-duplex and the transmission from the source and from the relay is assumed to be timeorthogonal. We choose QPSK as the modulation scheme as it has many practical applications and also offers decent performance for a reasonably low complexity. We assume full channel state information (CSI) at relay and the destination and perform coherent demodulation in

\footnotetext{
${ }^{1}$ Part of the work presented in this chapter appears in [56]
} 


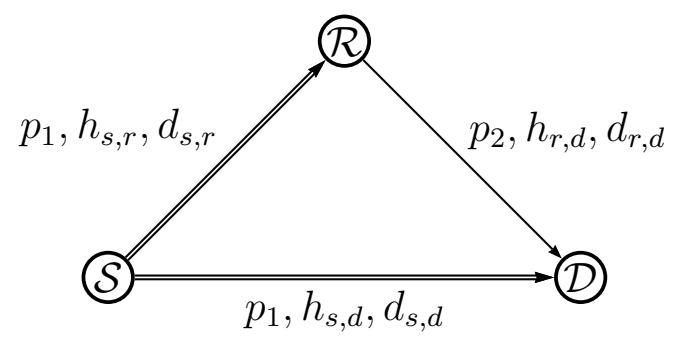

Figure 3.1: System model for a three terminal cooperative relay network.

this chapter and also when we use DF in the next chapter. The channel is modeled as a frequency non-selective one, where fading affects all the frequencies in the same way, with Rayleigh fading, where the fading amplitudes are Rayleigh distributed. As shown in Fig. 3.1, the channel gain between the source and destination is denoted by $h_{s, d}$, the channel gain between the source and the relay by $h_{s, r}$ and that between relay and destination by $h_{r, d}$. The channel gains are complex Gaussian with zero mean and unit variance and the fading amplitude $|h|$ is Rayleigh distributed. The distance between the source and the destination, denoted by $d_{s, d}$, is normalized to one as the relay is moved in a two dimensional plane. The distances between the source and relay and that between the relay and destination are represented as $d_{s, r}$ and $d_{r, d}$ respectively.

In our thesis we have used the term "Phase" to mean time slot and should not be confused with phase related to power or electricity. Because the system is half duplex, the transmission is two phased:

Phase 1 - The source transmits with a certain power, $p_{1}$ (indicated by the double lines in Fig. 3.1) and due to the broadcast nature of wireless communication, the relay as well as the destination receives a copy of it.

Phase 2 - The relay forwards the received signal using either AF (or DF in the next chapter) protocol using power $p_{2}$ (indicated by the single line in Fig. 3.1). This signal uses a path that is independent from the one used in Phase 1 and in doing so it also offers diversity gain. The two copies of the signals are then combined using maximal ratio combining and decoded. 
To facilitate the determination of optimal power division between the two phases, we constrained the total power to 1 . This is summarized in (3.1) as,

$$
\mathcal{P}_{\text {Total }}=p_{1}+p_{2}
$$

with $\mathcal{P}_{\text {Total }}$ set to one so that we can effectively express $p_{2}$ as

$$
p_{2}=1-p_{1}
$$

To investigate the effect of the relay position on the overall performance, the pathloss is also taken into consideration. We use a pathloss exponent of 3.6 and fix the noise power, $\sigma^{2}$ to $5 \times 10^{-15}$ Watts as used in [57]. A constant $k$ is introduced to flexibly scale the predetermined received SNR. We would consider a predetermined received SNR of 10dB and sometimes $20 \mathrm{~dB}$ throughout the thesis. From Fig. 2.1, for PSK modulation, where the signal space dimension is $K=2$, and the basis functions $f_{1}(t)=\sqrt{\frac{2}{T}} \cos 2 \pi f_{c} t$ and $f_{2}(t)=\sqrt{\frac{2}{T}} \sin 2 \pi f_{c} t$, we can write the complex received signal $r$ as

$$
r=r_{1}+j r_{2}
$$

where $r_{1}$ and $r_{2}$ are defined in (2.11). So for the transmitted symbol $b$ and pathloss coefficient $\alpha$, the received signal $r$ can be expressed as

$$
r=\frac{\sqrt{p} h k b}{d^{\alpha}}+n
$$

In (3.4), $h$ represents the complex channel gain or the fading coefficient and $n$ the complex AWGN noise with the power spectral density $N_{0}$ and we choose the pathloss coefficient $\alpha=3.6$. The distance between the source and the destination is quantified by $d$, normalized to one in our case and $p$ denotes the transmit power for that particular transmission phase. We evaluate the performance over a $3 \times 3$ unit grid, with the source at $(-0.5,0)$ and the destination placed at $(0.5,0)$. The relay can be placed anywhere within the grid.

\section{Direct Communication using Our Model}

We will now see first, how a system where the receiver changes distance with respect to the transmitter behaves, under a fixed received SNR. We will use the model described 
above in (3.4) to evaluate the system with the distance between the transmitter and receiver normalized to one. We have closed form expressions for the average probability of error for binary phase shift keying $[40,38]$ in a slow flat fading channel given by

$$
P_{b}=\frac{1}{2}\left[1-\sqrt{\frac{\Gamma}{1+\Gamma}}\right] .
$$

We know that the bit error probability for QPSK is same as BPSK and $\Gamma$ is the average received SNR in a fading channel so that we can use (3.5) for our case. But in our case the effect of distance also needs to be considered and here that effect can be grouped together with the noise power. We proceed to find the performance of direct communication with changing distance. In Fig. 3.2 we plot the analytical performance as well as the simulated performance for this setup.

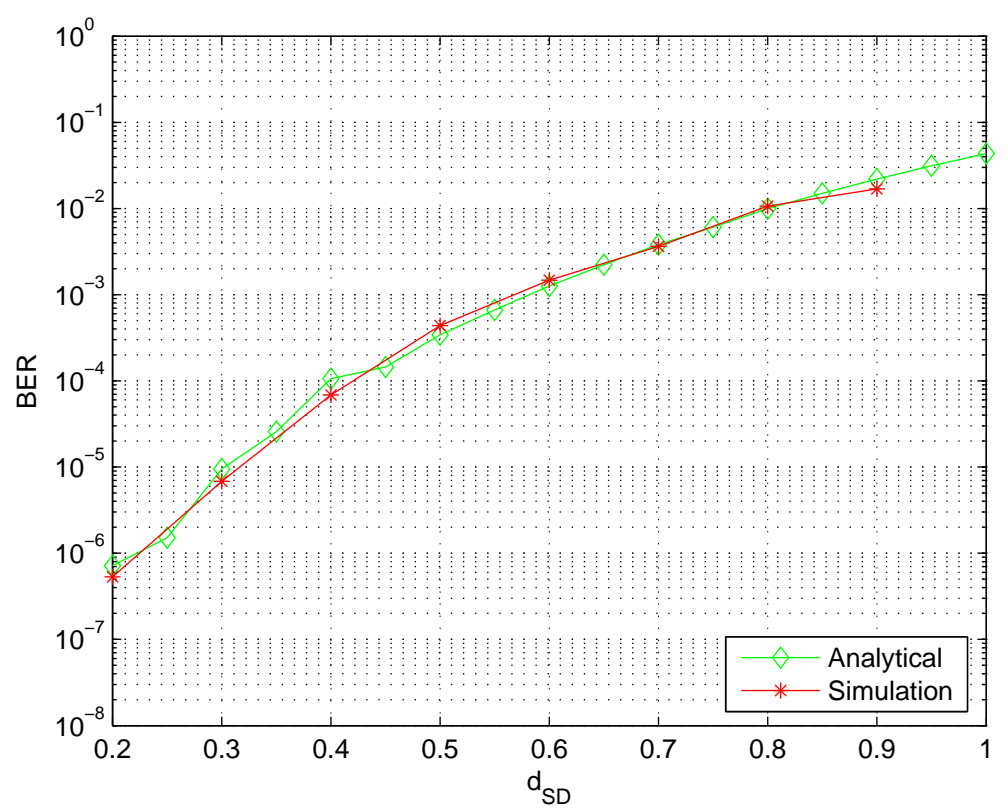

Figure 3.2: Analytical and simulation results for direct source to relay communication using distance model with received SNR of $10 \mathrm{~dB}$. The $\mathrm{x}$-axis represents the transmitter to receiver distance.

The $\mathrm{x}$-axis in Fig. 3.2 represents the transmitter to receiver distance. We can see from Fig. 3.2 that the performance predictably deteriorates as the distance of the receiver from the source increases. We also note that the performance is considerably better when the receiver is nearer to the source. This is also a pointer that if we place a relay in an intermediate 
position the individual distances between the terminals would reduce and there could be performance gains of the overall system. And this is the guiding beacon of our thesis.

\subsection{Performance Improvement Offered by Amplify and Forward}

The simplicity of amplify and forward and its ease of implementation has lead to a lot of analysis of its various performance metrics. In this technique the relay almost acts as a repeater. The signal received at the relay in Phase 1, from the source, is scaled and then retransmitted towards the receiver in Phase 2. We have assumed a total transmit power constraint, so according to (3.2) in Phase 2 the relay uses the portion of power left over from the first phase. We would proceed to formulate the instantaneous combined received $\mathrm{SNR}, \gamma_{\mathrm{AF}}$ for a predetermined received SNR which is used to calculate the probability of bit

error. In Phase 1 the relay and the destination receive signals as given by (3.6) and (3.7) respectively:

$$
\begin{aligned}
r_{s, r} & =\frac{\sqrt{p_{1}} k h_{s, r} b}{d_{s, r}^{\alpha}}+n_{s, r} \\
r_{s, d} & =\frac{\sqrt{p_{1}} k h_{s, d} b}{d_{s, d}^{\alpha}}+n_{s, d} .
\end{aligned}
$$

In this thesis we have considered a pathloss coefficient $\alpha=3.6$. The relay here does not detect or demodulate the received noisy signal but before transmitting in Phase 2, the relay scales $r_{s, r}$ by multiplying it by $\beta$, which is given by,

$$
\beta=\frac{1}{\left\|r_{s, r}\right\|}
$$

The purpose of this is to normalize the received power in the signal being transmitted from the relay, so that we can control the transmit power. At the end of Phase 2 , the destination receives a noisy copy of the signal from the relay given by

$$
\begin{aligned}
r_{r, d} & =\frac{\sqrt{p_{2}} k h_{r, d} \beta r_{s, r}}{d_{r, d}^{3.6}}+n_{r, d} \\
& =\frac{{\sqrt{p_{1} p_{2}} k^{2} \beta h_{s, r} h_{r, d} b}_{d_{s, r}^{3.6} d_{r, d}^{3.6}}+\frac{\sqrt{p}_{2} k \beta h_{r, d} n_{s, r}}{d_{r, d}^{3.6}}+n_{r, d}}{}
\end{aligned}
$$


We now form the decision statistic as shown in (3.11) and from that derive the instantaneous SNR. To achieve this, the signals $r_{s, d}$ and $r_{r, d}$ are individually multiplied by the complex conjugates of the respective channel gains, given by $h_{s, d}^{*}$ and $\left(h_{s, r} h_{r, d}\right)^{*}$ and then combined to give

$$
\begin{aligned}
d_{\mathrm{AF}} & =h_{s, d}^{*} r_{s, d}+\left(h_{s, r} h_{r, d}\right)^{*} r_{r, d} \\
& =h_{s, d}^{*}\left(\frac{\sqrt{p_{1}} k h_{s, d} b}{d_{s, d}^{3.6}}+n_{s, d}\right)+\left(h_{s, r} h_{r, d}\right)^{*}\left(\frac{\sqrt{p_{1} p_{2}} k^{2} \beta h_{s, r} h_{r, d} b}{d_{s, r}^{3.6} d_{r, d}^{3.6}}+\frac{\sqrt{p_{2}} k \beta h_{r, d} n_{s, r}}{d_{r, d}^{3.6}}+n_{r, d}\right) .
\end{aligned}
$$

We can simplify (3.11) as

$$
d_{\mathrm{AF}}=\frac{\sqrt{p_{1}} k\left|h_{s, d}\right|^{2} b}{d_{s, d}^{3.6}}+\frac{\sqrt{p_{1} p_{2}} k^{2} \beta\left|h_{s, r} h_{r, d}\right|^{2} b}{d_{s, r}^{3.6} d_{r, d}^{3.6}}+h_{s, d}^{*} n_{s, d}+\frac{\sqrt{p_{2}} k \beta h_{s, r}^{*}\left|h_{r, d}\right|^{2} n_{s, r}}{d_{r, d}^{3.6}}+\left(h_{s, r} h_{r, d}\right)^{*} n_{r, d} .
$$

From (3.12), we express the instantaneous SNR as

$$
\gamma_{\mathrm{AF}}=\frac{\left(\frac{\sqrt{p_{1}} k\left|h_{s, d}\right|^{2}}{d_{s, d}^{3.6}}+\frac{\sqrt{p_{1} p_{2}} k^{2} \beta\left|h_{s, r} h_{r, d}\right|^{2}}{d_{s, r}^{3.6} d_{r, d}^{3,6}}\right)^{2}}{\left(\left|h_{s, d}\right|^{2}+\frac{p_{2} k^{2} \beta^{2}\left|h_{s, r}\right|^{2}\left|h_{r, d}\right|^{4}}{d_{r, d}^{7.2}}+\left|h_{s, r} h_{r, d}\right|^{2}\right) N_{0}} .
$$

Using (3.2), (3.13) can be written as

$$
\gamma_{\mathrm{AF}}=\frac{\left(\sqrt{p_{1}} k\left|h_{s, d}\right|^{2}+\frac{\sqrt{\left(p_{1}-p_{1}^{2}\right)} k^{2} \beta\left|h_{s, r} h_{r, d}\right|^{2}}{d_{s, r}^{3.6}\left(1-d_{s, r}\right)^{3.6}}\right)^{2}}{\left(\left|h_{s, d}\right|^{2}+\frac{\left(1-p_{1}\right) k^{2} \beta^{2}\left|h_{s, r}\right|^{2}\left|h_{r, d}\right|^{4}}{\left(1-d_{s, r}\right)^{7.2}}+\left|h_{s, r} h_{r, d}\right|^{2}\right) N_{0}} .
$$

Thus the average probability of bit error is

$$
P_{b}=\mathrm{E}\left[Q\left(\sqrt{\gamma_{\mathrm{AF}}}\right)\right]
$$

\subsubsection{Power Optimization in Collinear Case}

Considering the source and destination at unit distance apart, the relay in between but collinear to the source and destination, we simulate to evaluate the performance of this system. Initially we divide the transmit power equally between the two phases ${ }^{1}$. We compare the simulated performance with the semi-analytical ${ }^{2}$ result obtained from (3.15).

\footnotetext{
${ }^{1}$ Power allocations will be denoted with the transmit power in phase one first and then that in Phase 2 second within first brackets. For example, if 10 percent of the power is sent in Phase 1 and the rest in Phase 2 , it will be represented as (10-90).

${ }^{2}$ By semi-analytical here we mean that the BER given by (3.15) is evaluated by replacing the expectation by time averaging $Q\left(\sqrt{\gamma_{\mathrm{AF}}}\right)$.
} 
As can be seen from Fig. 3.3 the semi-analytical and simulation results match, confirming the validity of (3.15).

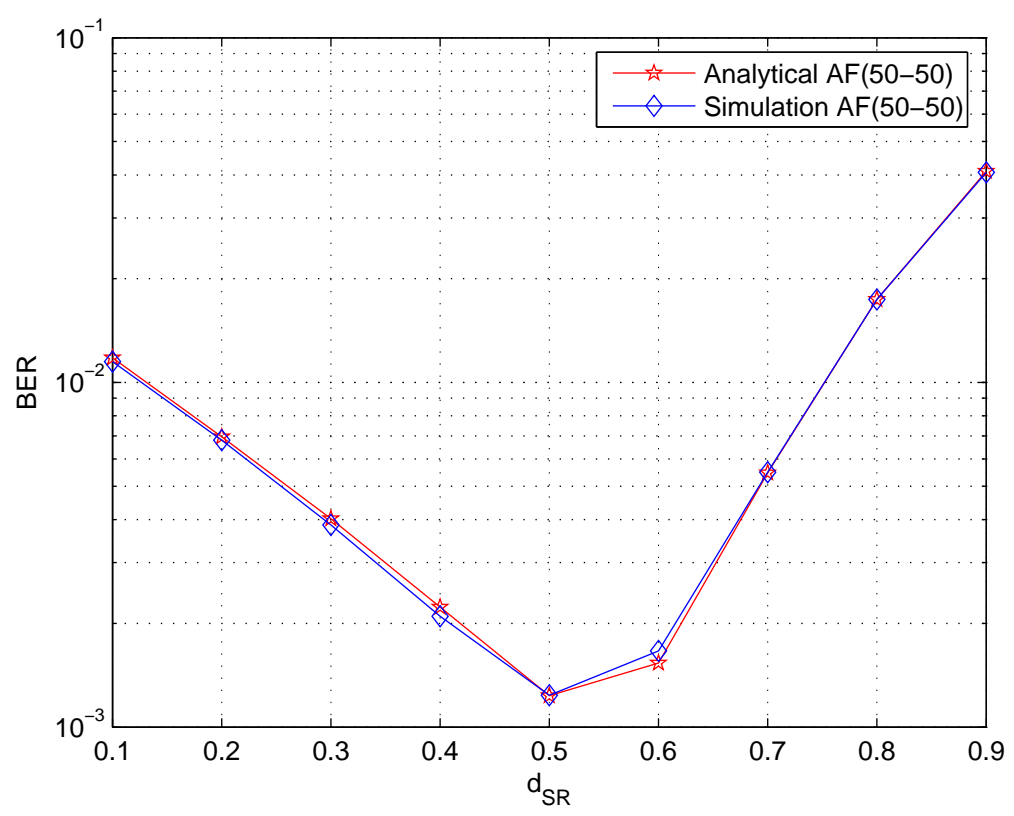

Figure 3.3: Comparison of semi-analytical and simulated BER performance of AF . The transmit power is equally divided in both the phases.

Our next goal is to check how the system responds to various kinds of power allocated in Phase 1 and Phase 2. We check some arbitrary power allocations, starting from relatively low power in Phase 1 to relatively more power in Phase 2. The family of curves for a received SNR of $10 \mathrm{~dB}$ at the destination, are plotted in Fig. 3.4 and Fig. 3.5 using semi-analytical method and simulation respectively.

The fact that power allocation plays a crucial role in determining the overall system performance becomes clear from Fig. 3.4 and Fig. 3.5. The best performance is achieved around the midway region, though it may vary slightly for different power allocations. No single power allocation has the best performance throughout as the relay moves between the source and destination. From Fig. 3.5 it is also seen that some power allocations perform worse than direct communication. The next logical step to improve system performance would be to optimally allocate the powers in the different transmission phases depending on the position of the relay relative to the source and destination. It is evident that if we are able to optimize the instantaneous phase powers to maximize $\gamma_{\mathrm{AF}}$ given by (3.14) and use the 


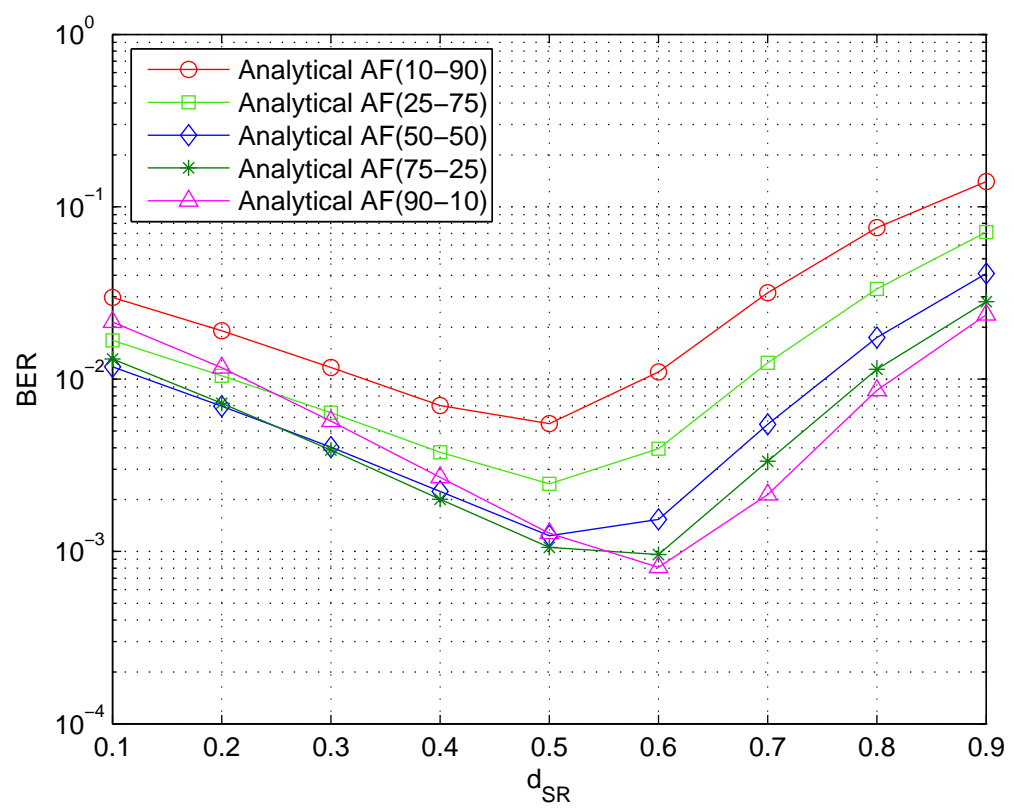

Figure 3.4: Comparison of semi-analytical BER performance of AF with various different power allocations in Phase 1 and Phase 2.

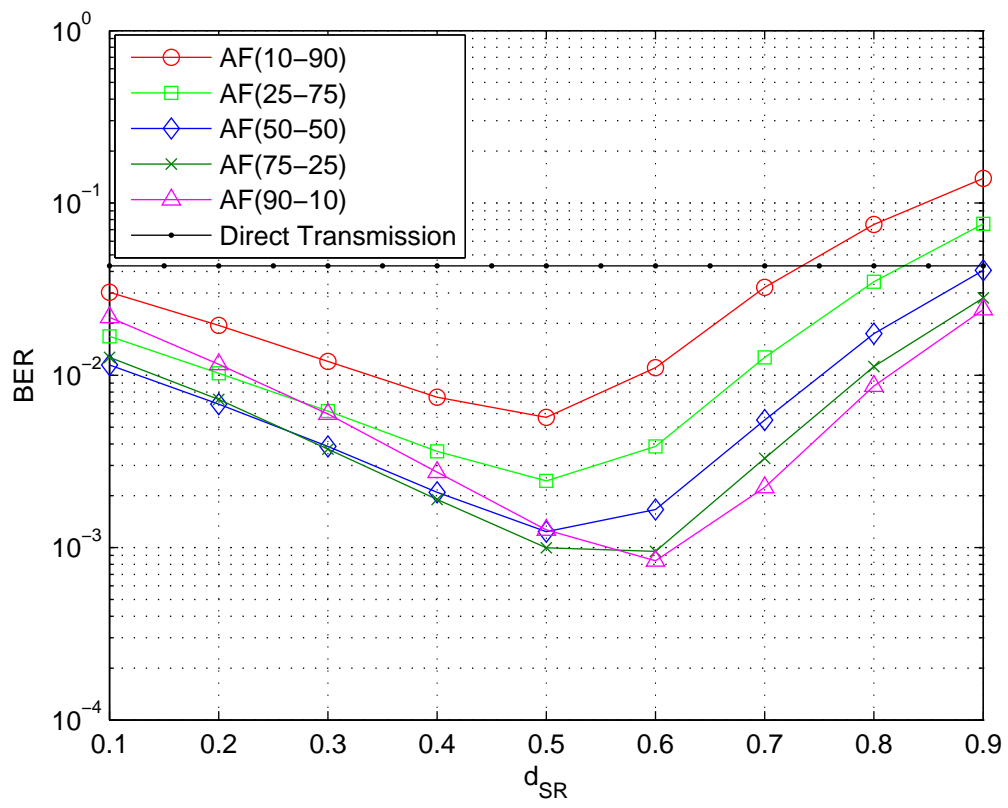

Figure 3.5: Comparison of simulated BER performance of AF with various different power allocations in Phase 1 and Phase 2 and direct communication.

optimized powers, we would be able to extract almost optimal BER performance from our system. We numerically optimize (3.14) for $p_{1}$, the power in Phase 1, using Quasi-Newton 
method [58, 59], under the total power constraint given by (3.1).

The optimal power allocation thus obtained are then utilized in resimulating the system and the performance achieved is shown in Fig. 3.6. The fact that the optimal power allocation outperforms the arbitrary ones all through is clearly understandable from the Fig. 3.6. In Fig. 3.6 we also plot the performance of direct communication and notice that while the optimal power allocation scheme is throughout better than this, there are some arbitrary power allocations which perform worse than the direct communication at certain locations. This happens especially when the transmit power in Phase 1 is low and the relay is much closer to the destination than the source. This can be explained as follows. As the relay moves away from the source, its performance gets worse and this coupled with low power transmission in Phase 1 only compounds the problem leading to bad performance.

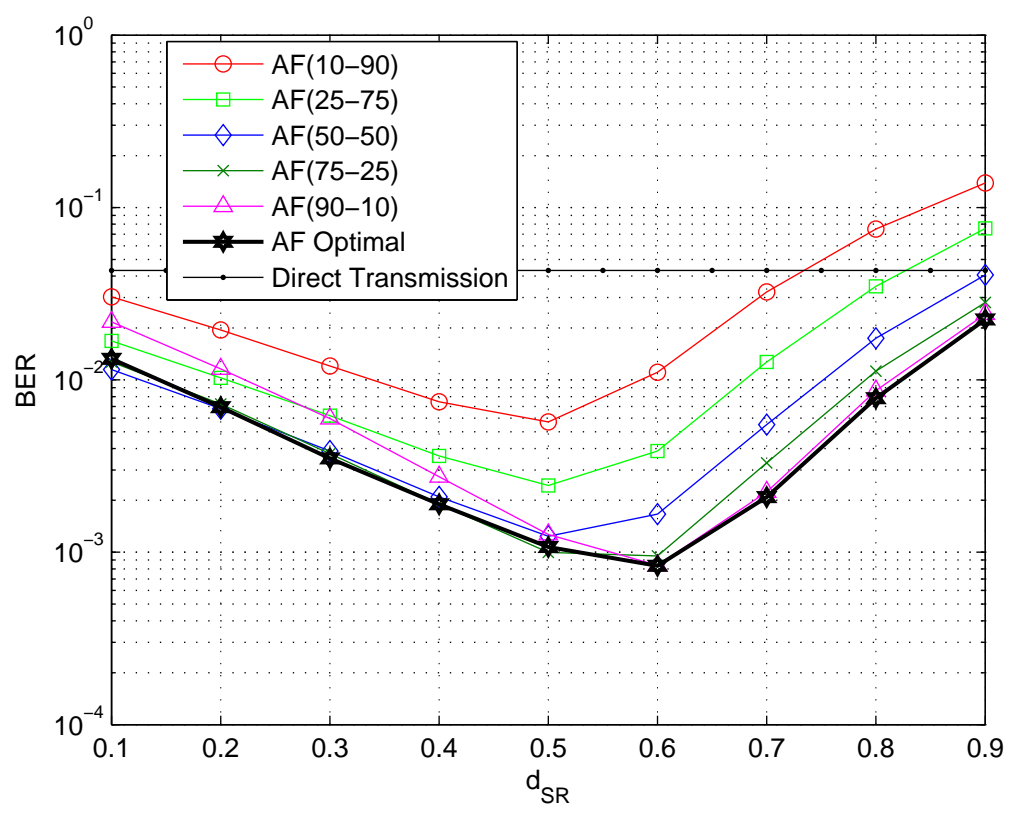

Figure 3.6: Comparison of simulated BER performance of AF with various different power allocations in Phase 1 and Phase 2, direct communication, and AF with optimal power allocation.

It would be interesting to see how the system behaves when we change the received SNR. The effects of this when we change the received SNR from 10dB to $20 \mathrm{~dB}$ is shown in Fig. 3.7 and compared with direct transmission. Here we observe that the the performance at the higher SNR is better throughout and also the amount by which the system is better than 
direct communication is more for the higher SNR. The throughput performance of AF at $10 \mathrm{~dB}$ and $20 \mathrm{~dB}$ received SNRs at destination is plotted to investigate the system behavior and also compared with direct communications at those SNRs. In this thesis for a normalized rate $R$ system with packet size $M$, we define throughput as

$$
T_{p}=R(1-B E R)^{M} \quad \text { bits/timeslot. }
$$

Our system is half duplex so the rate is $R=0.5$ and the packet size $M=120$ bits/timeslot. In DF the packet size $M=14$ and is kept small to increase the probability of delivering a packet correctly. Whenever AF and DF are compared the packet size of both are kept at $M=14$ for fairness. The Fig. 3.8 shows that the throughput of the higher SNR is better throughout, specially so when compared to the lower SNR at regions near the source or the destination. The dramatic increase in throughput at $20 \mathrm{~dB}$ received SNR is the result of considerably better BER performance at 20dB received SNR, as seen in Fig. 3.7. Higher received SNR requires more power to be injected into the system, which in turn helps in reducing the effects of fading and additive noise and thus delivering the signal with more accuracy, increasing throughput and improving BER.

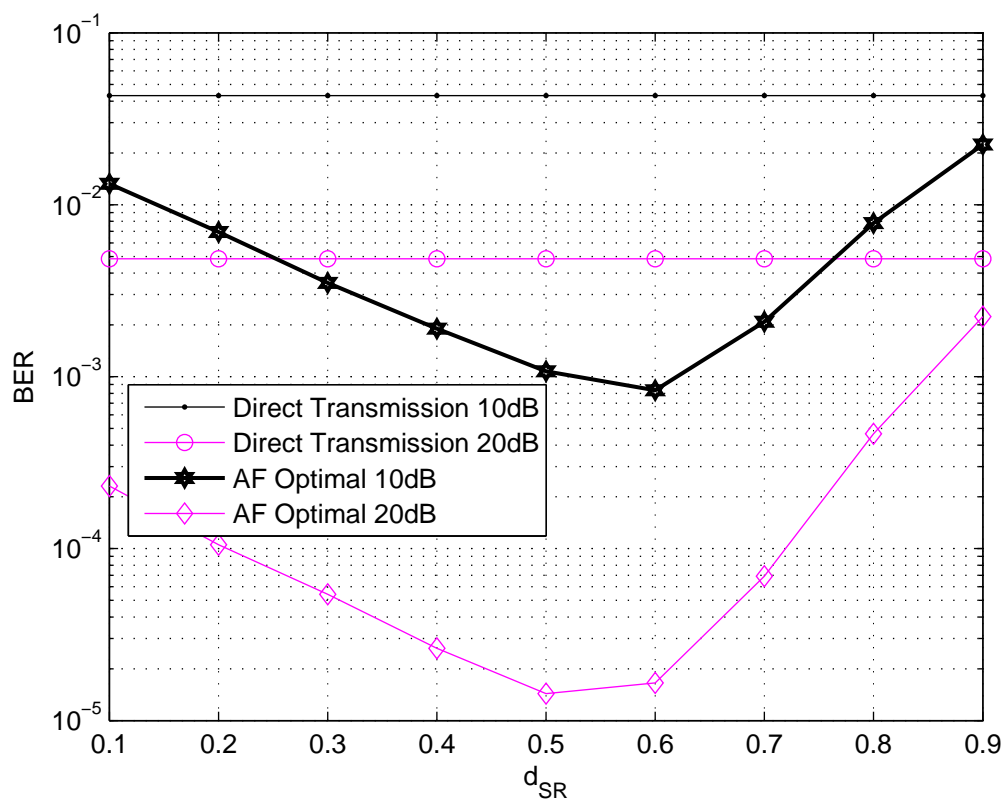

Figure 3.7: Comparison of simulated BER performance of AF with optimal power allocation and direct communication for received SNRs of $10 \mathrm{~dB}$ and $20 \mathrm{~dB}$. 


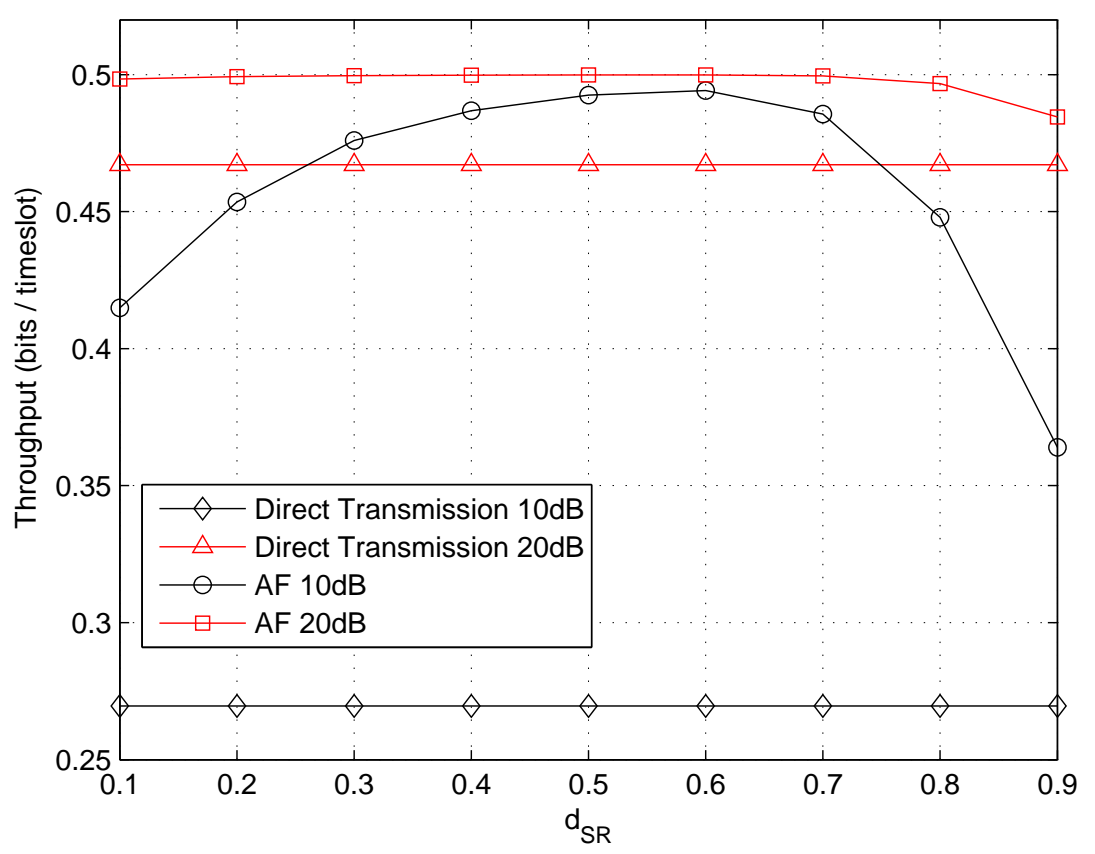

Figure 3.8: Comparison of the throughput of AF with optimal power allocation and direct communication for received SNRs of $10 \mathrm{~dB}$ and $20 \mathrm{~dB}$.

\subsubsection{Power Optimization Over a Grid and Area of Interest}

Having seen how the system behaves when the relay is collinear to the source and destination it would be interesting to see how the system performs when the relay is free to move over a two dimensional grid in the same plane as the source and destination. We consider a $3 \times 3$ grid with the source at $x=-0.5, y=0$ and the destination at $x=0.5, y=0$. Fig. 3.9 shows the grid with the source and destination pointed out. The relay can move over this region but for computational purposes we assume the relay to be in any of the intersecting grid points. Depending on the relative distance of the relay from the source and destination there would be an optimum power allocation scheme for each of these positions. Using (3.13) and (3.15) and using Quasi-Newton optimization we obtain the specific power allocations for each grid point as is shown in Fig. 3.10. Now armed with the knowledge of optimal power allocation we simulate the performance of the system over the grid and plot the BER performance in Fig. 3.11.

Fig. 3.11 shows the BER performance of the system which also shows that the best performance is achieved if the relay is placed about halfway between the source and destina- 


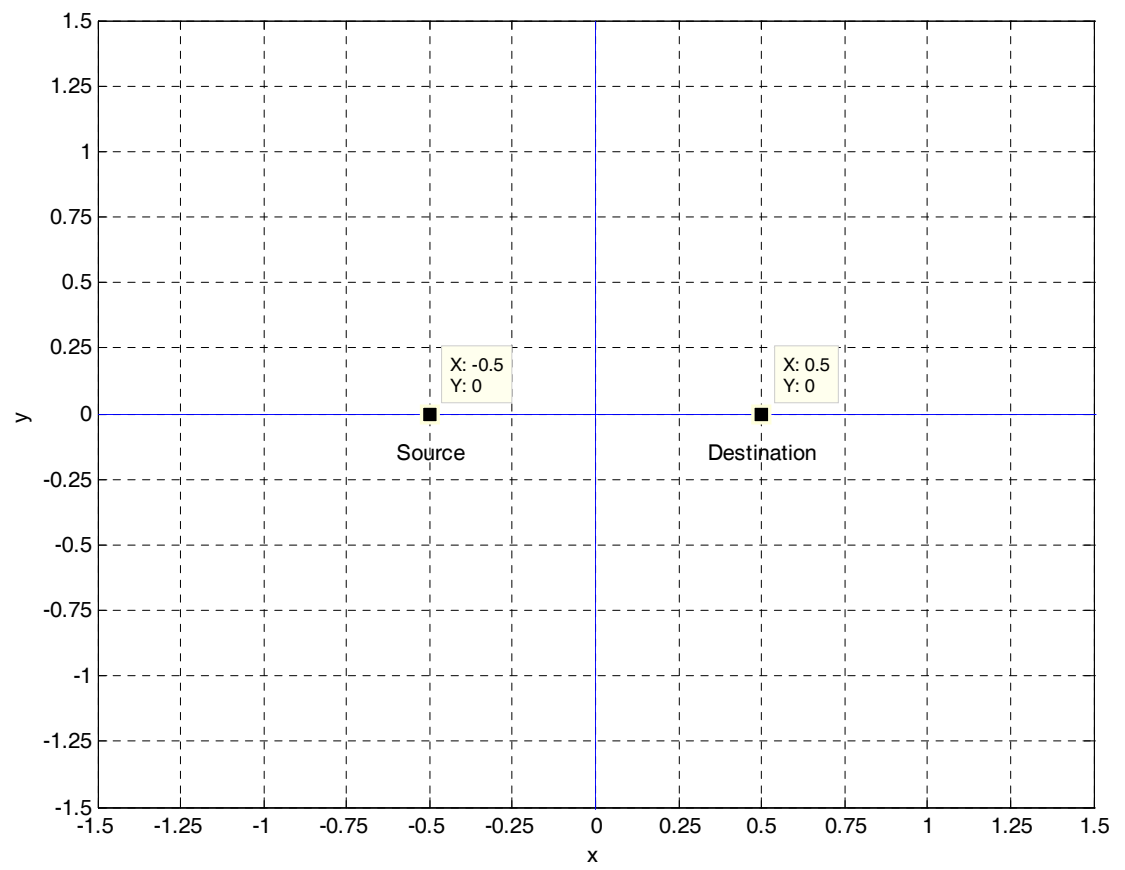

Figure 3.9: Layout of the grid with the source at $\mathrm{x}, \mathrm{y}=(-0.5,0)$ and destination at $\mathrm{x}, \mathrm{y}=$ $(0.5,0)$.

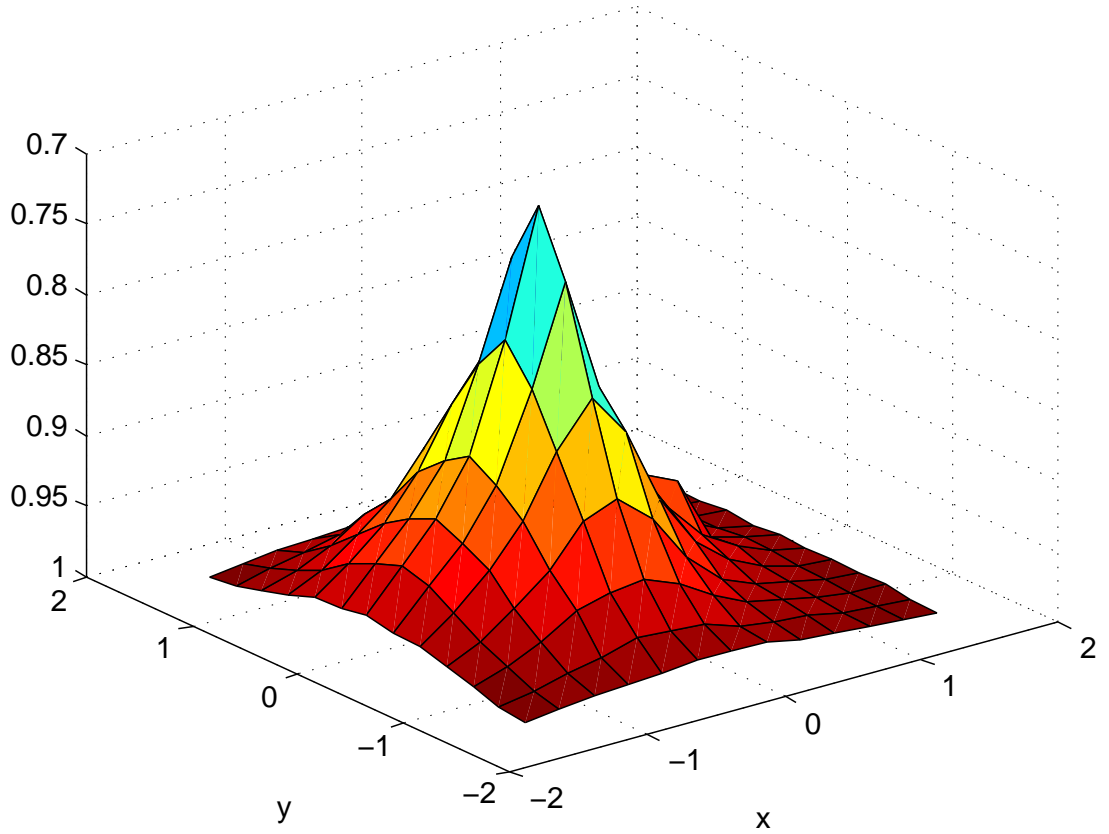

Figure 3.10: Phase 1 power $p_{1}$ for AF . The received SNR is $10 \mathrm{~dB}$. Source at $\mathrm{x}, \mathrm{y}=(-0.5,0)$ and destination at $\mathrm{x}, \mathrm{y}=(0.5,0)$. The $\mathrm{z}$ axis represents the optimal value of $p_{1}$. 


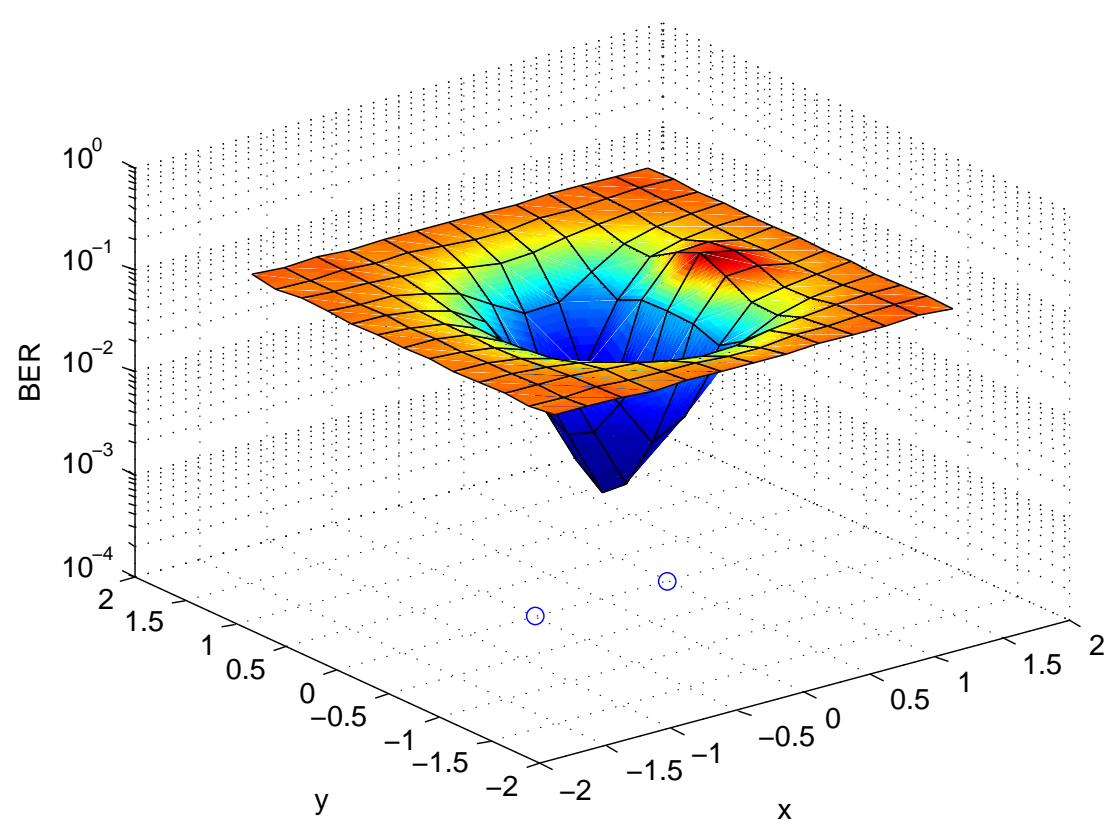

Figure 3.11: BER performance of AF over the grid. The received SNR is 10dB. Source at $\mathrm{x}, \mathrm{y}=(-0.5,0)$ and destination at $\mathrm{x}, \mathrm{y}=(0.5,0)$.

tion. There is degradation of performance as the relay moves farther away from the source or destination. So there would be some regions where the performance is worse than the direct communication. To figure this out we plot the performance of direct communication on the same plot as Fig. 3.11, which is a plane of same BER. This is clearly seen in Fig. 3.12, where the gray plane represent the performance of direct communication and, parts of the power optimized BER surface plot above it denotes areas where AF is worse than direct communication. In Fig. 3.13, to identify the regions where AF with optimal power allocation outperforms direct communication, we look at Fig. 3.12 from the top.

Fig. 3.13, which is the top view of Fig. 3.12, points to fact that significant improvement over direct transmission is achieved over a wide area of the grid we considered. The inner lighter colored, cardioid shaped area represents the area where relaying is useful in AF.

The portion of total power used for transmission in Phase 1 given by $p_{1}$ is plotted in Fig. 3.10, which shows how $p_{1}$ changes with the position of the relay. We see that in the grid we considered, points having better BER performance than others also use comparatively lesser amount of power in Phase 1 than points with worse BER performance. Depending on the received SNR there should be changes in the system performance. We check the 


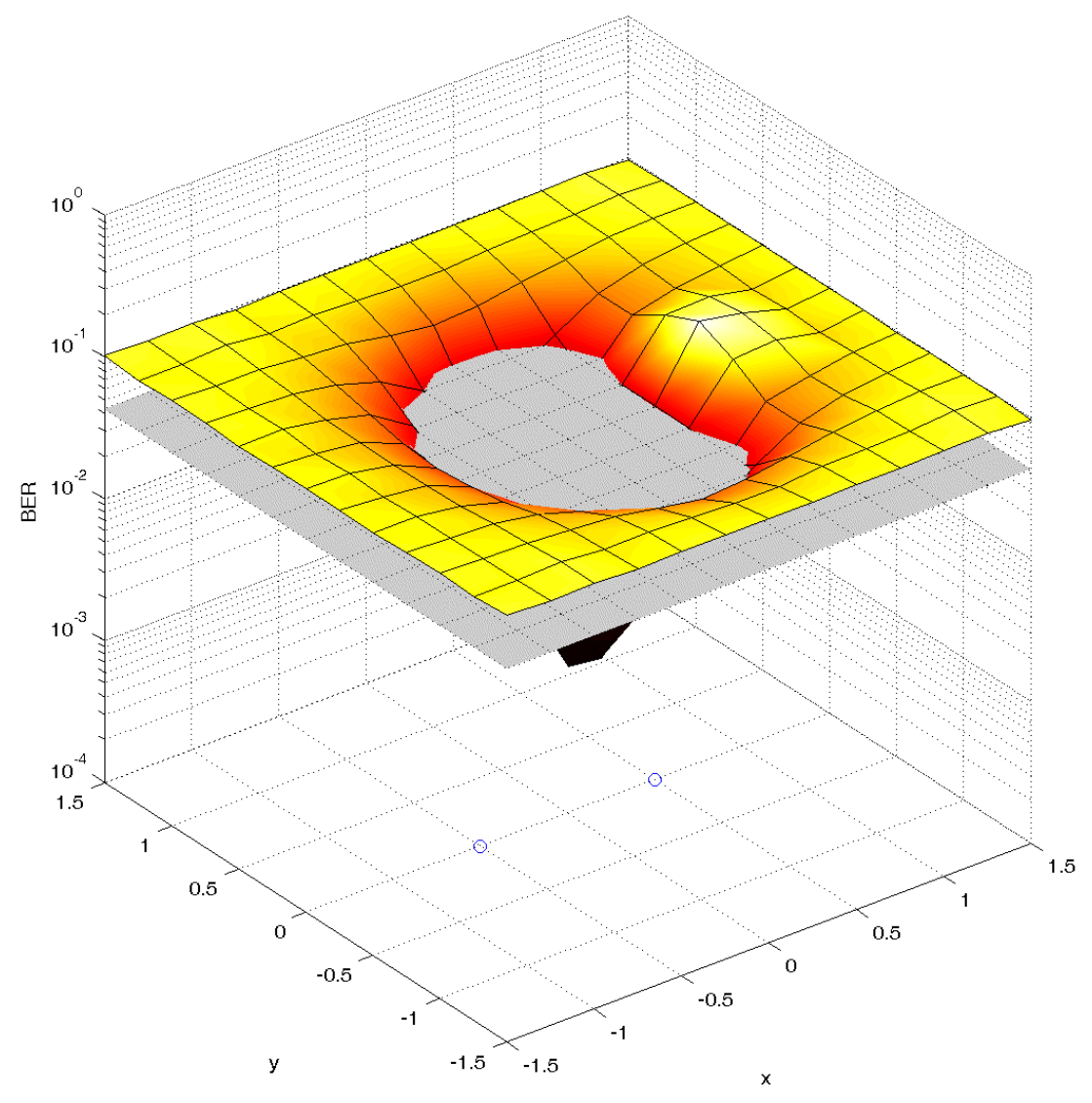

Figure 3.12: BER performance of AF and direct communication over the grid . The received SNR at destination is $10 \mathrm{~dB}$. Source at $\mathrm{x}, \mathrm{y}=(-0.5,0)$ and destination at $\mathrm{x}, \mathrm{y}=(0.5,0)$.

system performance with optimal power allocation at $10 \mathrm{~dB}$ and $20 \mathrm{~dB}$ received SNR. The performance of the system with $20 \mathrm{~dB}$ received SNR is markedly better than the $10 \mathrm{~dB}$ received SNR, specially midway between the source and the destination.

We will define the area where our system outperforms direct communication as the "area of interest" and the way this area changes with increasing SNR would be interesting to learn. To visualize this, we simulate the performance of the power optimized system and that of direct communication, for $10 \mathrm{~dB}$ and $20 \mathrm{~dB}$ received SNR, and compare the region where relaying provides performance gains. In Fig. 3.15 it can be clearly seen that for the higher SNR there is a significant increase in the size of the area under discussion. This indicates that the relative amount by which the performance of the relay communication improves, at least in the area of interest, is more than that for direct communication. 


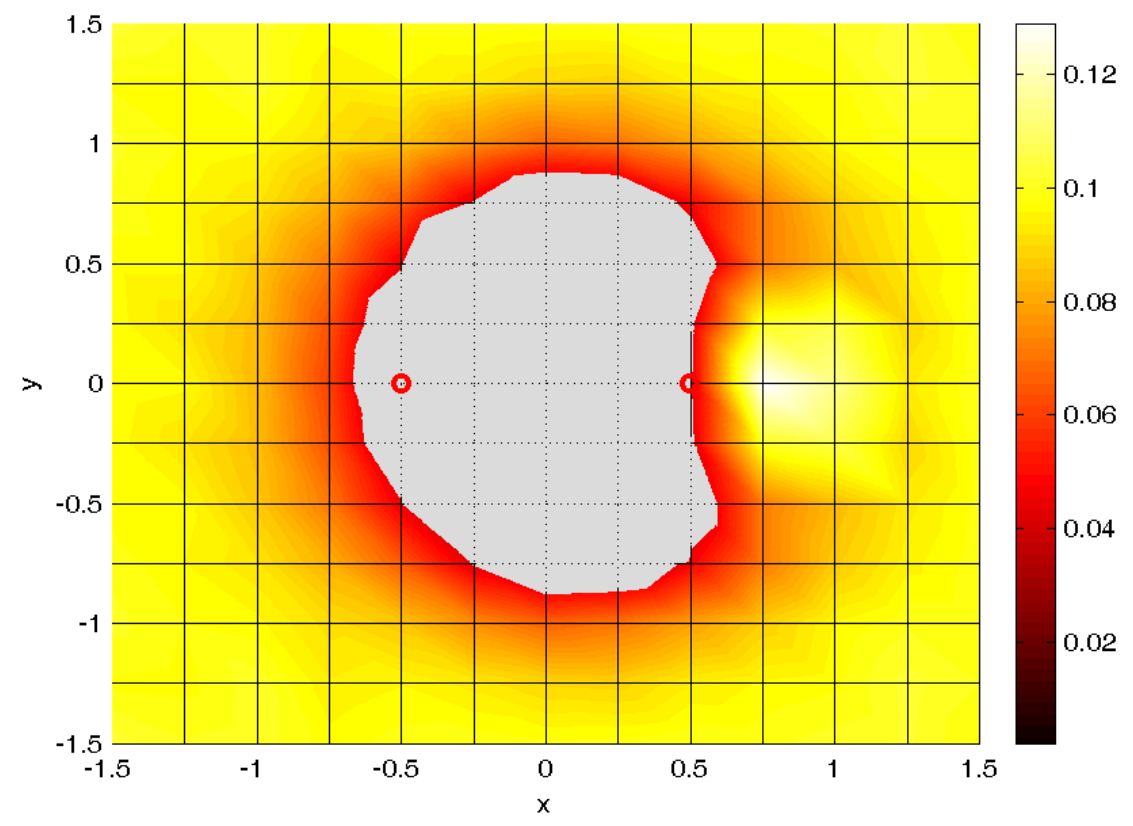

Figure 3.13: The inner lighter area represents the region for relaying in AF. The received $\mathrm{SNR}$ is $10 \mathrm{~dB}$. Source at $\mathrm{x}, \mathrm{y}=(-0.5,0)$ and destination at $\mathrm{x}, \mathrm{y}=(0.5,0)$.

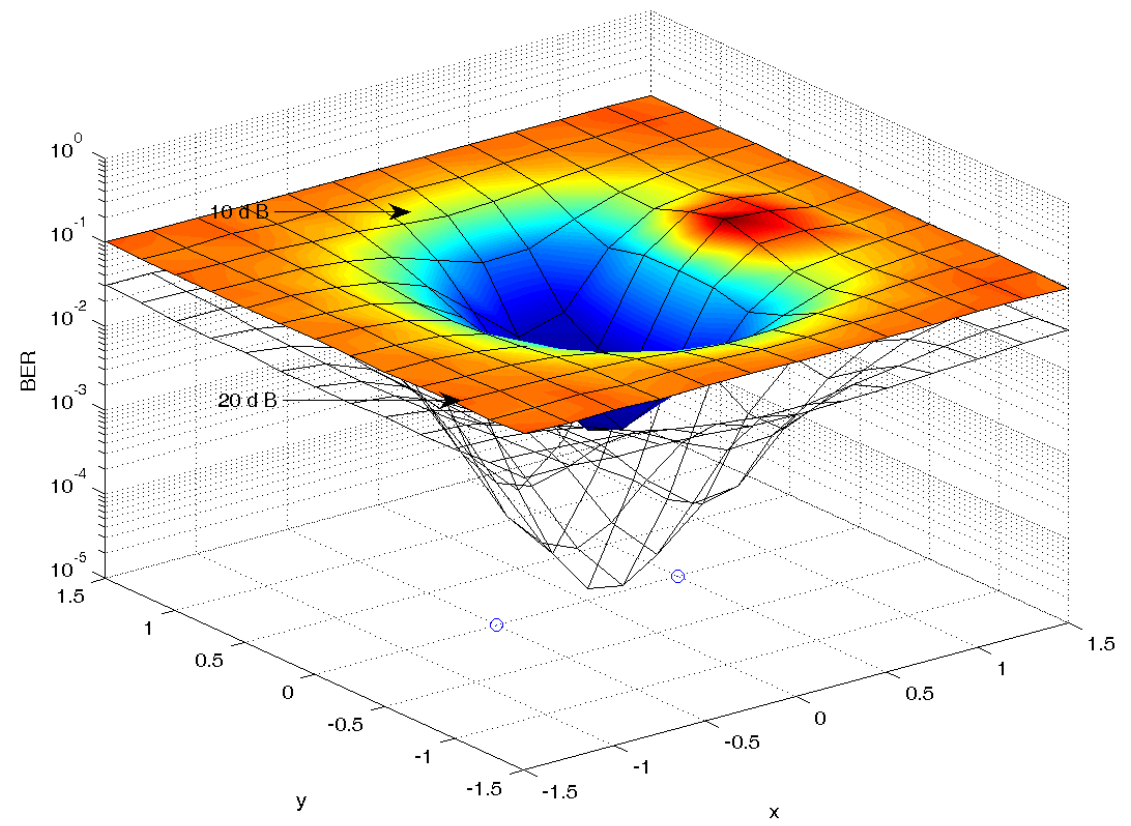

Figure 3.14: Comparison of BER performances of AF with received SNRs 10dB and 20dB. Source at $\mathrm{x}, \mathrm{y}=(-0.5,0)$ and destination at $\mathrm{x}, \mathrm{y}=(0.5,0)$. 

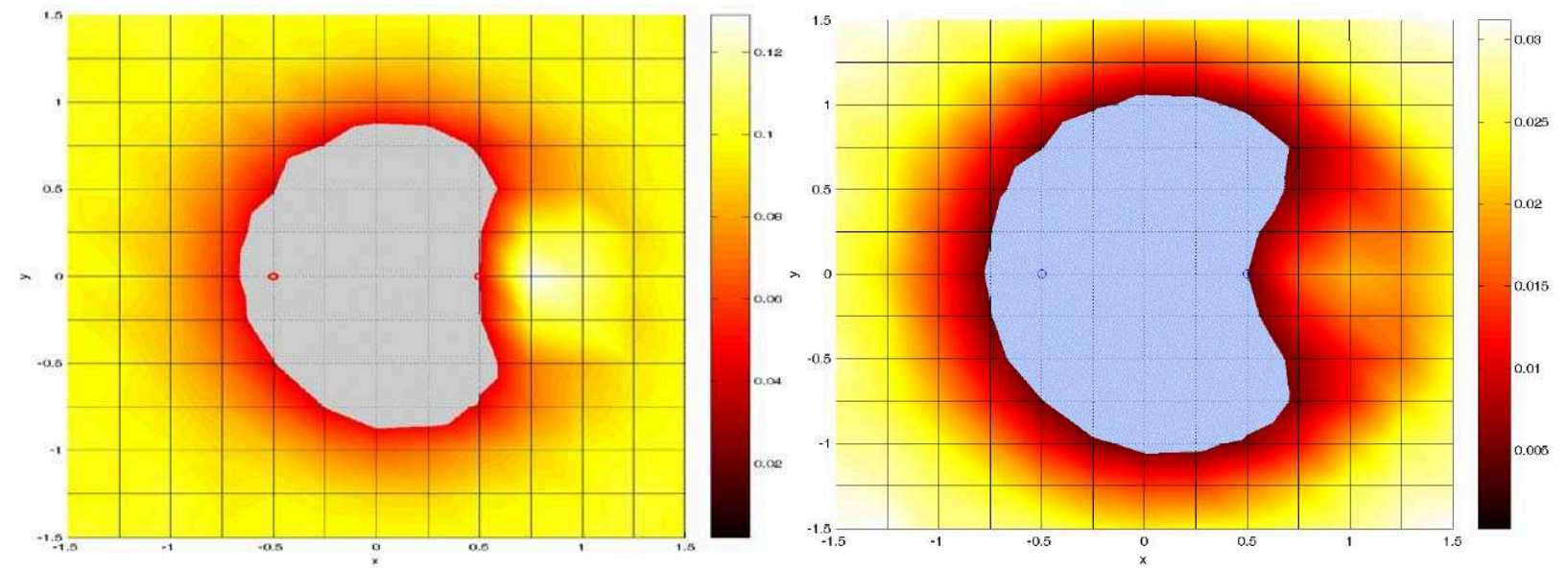

Figure 3.15: The inner lighter areas represent the region for relaying in coherent environment using AF at the relay. The received SNR for the left hand plot is $10 \mathrm{~dB}$ and that for the right hand one is $20 \mathrm{~dB}$. Source at $\mathrm{x}, \mathrm{y}=(-0.5,0)$ and destination at $\mathrm{x}, \mathrm{y}=(0.5,0)$.

\subsection{Conclusion}

Even being a simple technique itself, amplify and forward provides improvement of performance over direct communication in certain scenarios, specially when the relay is midway between the source and the receiver, though the best performance point may vary slightly with different power allocations. We also see that this performance is further enhanced by the use of optimal power allocation. We conclude that, though we can place the relay anywhere in our grid, it makes practical sense to use AF and place the relay around the midway region between the source and destination, which in turn lies inside the region where relaying is relevant, because around that region our optimal power allocation scheme can extract the better performance of the system. It is also observed that with increasing SNRs the area of the region where relaying is relevant increases. 


\section{Chapter 4}

\section{Decode and Forward with Coherent Modulation}

\subsection{Introduction}

In this chapter we will evaluate the performance of the regenerative DF protocol and compare it with AF. The same system model as described in the previous chapter is used here, only the forwarding technique at the relay is different. As in the case of AF the relay receives the broadcast from the transmitter in Phase 1, but instead of simply scaling and forwarding the signal, the relay tries to decode the signal and then forwards it. We assume perfect cyclic redundancy code (CRC) in the source to relay link so that the relay can perform a CRC check on the received signal and forward only if it decodes correctly. Since we assume CRC, we use term decode and forward instead of detect and forward or demodulate and forward.

We can also consider the scenario where the relay forwards everything it decodes without ever checking whether the decoded data is accurate. Nowadays, with extreme miniaturization relay nodes have become smart and have at least some means of checking the verity of the received data. It is intuitive that if the relay forwards only correct data there would be at least some improvement of the performance of the system other than perhaps when the power allocated in Phase 1 is low. Using our system model we proceed to simulate the

\footnotetext{
${ }^{1}$ Part of the work presented in this chapter appears in [56]
} 
scenario where the relay forwards any received data when equal power is allocated to both the transmission phases but before that we would also look into a CRC code to get a feel of how a perfect CRC might work. We will use CRC-5, a relatively weak CRC with degree 5, to demonstrate what we should expect if, instead we use a stronger CRC with a generator polynomial of high degree. It would also be interesting to compare these results with the one where the relay forwards only correct data.

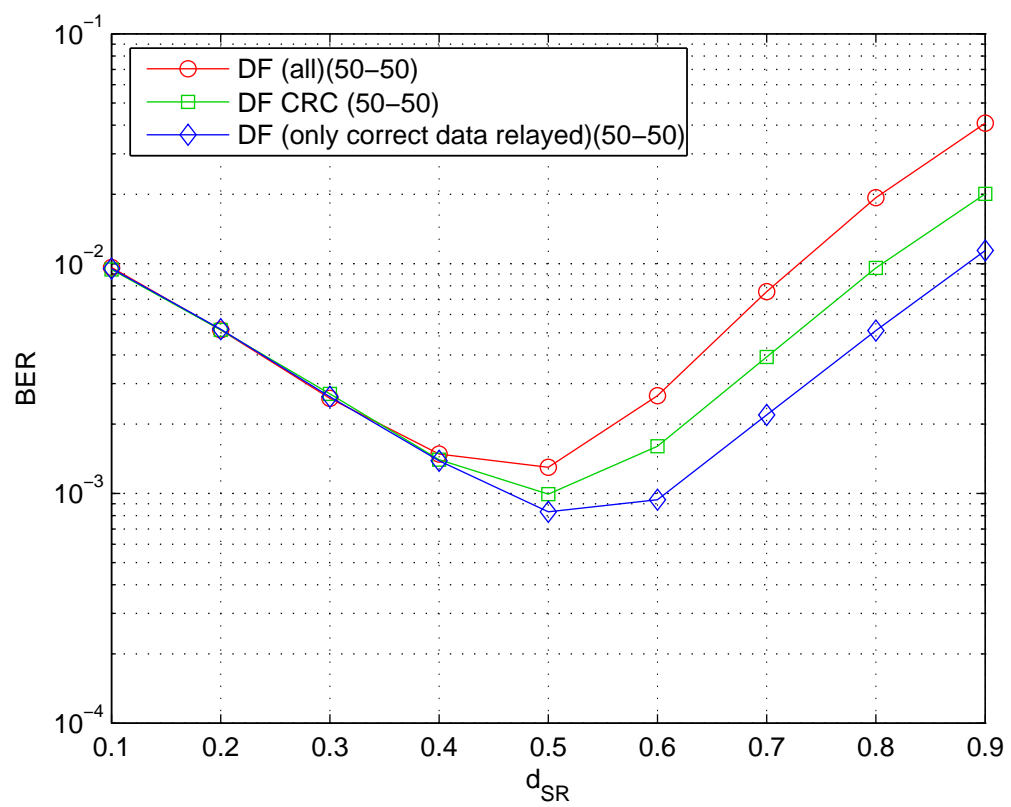

Figure 4.1: BER performances of the system when the relay forwards any received data, does a CRC-5 check before forwarding and when it forwards only correct data from the relay. Equal power is allocated to both the transmission phases. The received SNR at destination is $10 \mathrm{~dB}$.

As can be seen from Fig. 4.1 as long the relay is closer to the source and is almost always receiving correct data, the system performance for all three systems are almost the same. But as the relay moves away from the source, the performance of the system which forwards any received data degrades. The system with CRC checking performs better but since the CRC-5 polynomial used is of small degree there are errors that slips through and consequently the performance is not as good as that when the relay forwards only correct data. So we can see that a good CRC adds to the performance of the system as a whole and throughout the remainder of the thesis we will assume that in the case of DF the relay 
forwards only correct data.

\subsection{Performance Improvement Offered by Decode and Forward}

Here we would strive to see the how much the decode and forward technique improves the performance and also try to maximize the performance of the system. In our system the relay forwards only correct data, so in this case the quality of the source to relay link assumes importance. In Phase 1 the relay and the destination receives signals given by (3.6) and (3.7). We model the probability that relay decodes correctly as a Bernoulli random variable

$$
\eta \sim \operatorname{Bernoulli}(\rho)
$$

whose parameter $\rho$ is the probability of receiving a correct bit from the source at the relay. In Phase 2 the relay transmits with the power $p_{2}$, the portion of power left over from Phase 1 , given by (3.2). The signal received by the destination in Phase 2 can be expressed as

$$
r_{r, d}=\frac{\sqrt{p_{2}} k h_{r, d} \eta b}{d_{r, d}^{3.6}}+n_{r, d}
$$

where $\eta$ is the Bernoulli random variable discussed in (4.1). From (4.2) it can be seen that whenever the relay determines it has not decoded correctly, the first term is zero and the signal received at the destination is pure noise. But the destination has no way to figure that and hence it always listens to the relay even when the relay is silent. An immediate effect is that the performance of power optimized relaying does not converge to that of direct transmission when the relay is not actually forwarding. The two received signals from the source in Phase 1 and the relay in Phase 2 are then multiplied by the complex conjugate of their individual channel gains and then combined to form the decision statistic as shown in 


$$
\begin{aligned}
d_{\mathrm{DF}} & =h_{s, d}^{*} r_{s, d}+h_{r, d}^{*} r_{r, d} \\
& =h_{s, d}^{*}\left(\frac{\sqrt{p_{1}} k h_{s, d} b}{d_{s, d}^{3.6}}+n_{s, d}\right)+h_{r, d}^{*}\left(\frac{\sqrt{p_{2}} k h_{r, d} \eta b}{d_{r, d}^{3.6}}+n_{r, d}\right) \\
& =\frac{\sqrt{p}_{1} k\left|h_{s, d}\right|^{2} b}{d_{s, d}^{3.6}}+h_{s, d}^{*} n_{s, d}+\frac{\sqrt{p_{2}} k \eta\left|h_{r, d}\right|^{2} b}{d_{r, d}^{3.6}}+h_{r, d}^{*} n_{r, d} .
\end{aligned}
$$

From (4.3) we can write the instantaneous SNR as

$$
\gamma_{\mathrm{DF}}=\frac{\left(\frac{\sqrt{p_{1}} k\left|h_{s, d}\right|^{2}}{d_{s, d}^{3.6}}+\frac{\sqrt{p_{2}} k \eta\left|h_{r, d}\right|^{2}}{d_{r, d}^{3.6}}\right)^{2}}{\left(\left|h_{s, d}\right|^{2}+\left|h_{r, d}\right|^{2}\right) N_{0}} .
$$

Using (3.2) and noting that the source to destination distance is one, we can write (4.4) as

$$
\gamma_{\mathrm{DF}}=\frac{\left(\sqrt{p_{1}} k\left|h_{s, d}\right|^{2}+\frac{\sqrt{1-p_{1}} k \eta\left|h_{r, d}\right|^{2}}{d_{r, d}^{3.6}}\right)^{2}}{\left(\left|h_{s, d}\right|^{2}+\left|h_{r, d}\right|^{2}\right) N_{0}} .
$$

Similar to the AF case the probability of bit error is expressed as

$$
P_{b}=\mathrm{E}\left[Q\left(\sqrt{\gamma_{\mathrm{DF}}}\right)\right]
$$

\subsubsection{Power Optimization in Collinear Case}

In our system model we have considered the source and destination at unit distance apart. We simulate to evaluate the performance of the system when the relay is in between but collinear to the source and transmitter. To start with we divide the transmit power equally between the two phases ${ }^{1}$. We check the performance of this system with the semi-analytical result obtained from (4.6) where the expectation is replaced with time averaging.

Comparing the curves in Fig. 4.2 it can be seen that the simulation and analytical results match which verifies the equation (4.6).

As seen in the previous chapter the power allocation in the individual transmission phases plays a crucial role in determining the system performance. To show the dependence of performance on power allocation we plot the BER curves for a number of arbitrary power allocations for both simulated and semi-analytical method.

\footnotetext{
${ }^{1}$ Power allocations will be denoted with the transmit power in phase one first and then that in Phase 2 second within first brackets. For example, if 10 percent of the power is sent in Phase 1 and the rest in Phase 2 , it will be represented as (10-90).
} 


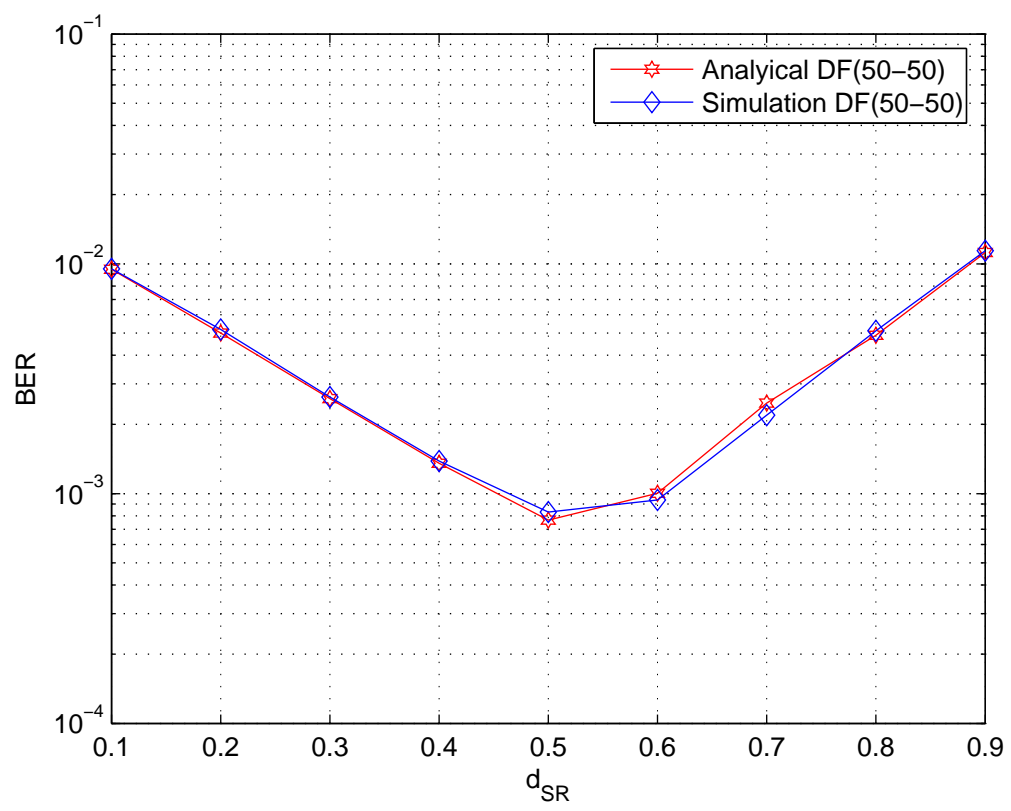

Figure 4.2: Comparison of semi-analytical and simulated BER performance of DF . The transmit power is equally divided in both the phases.

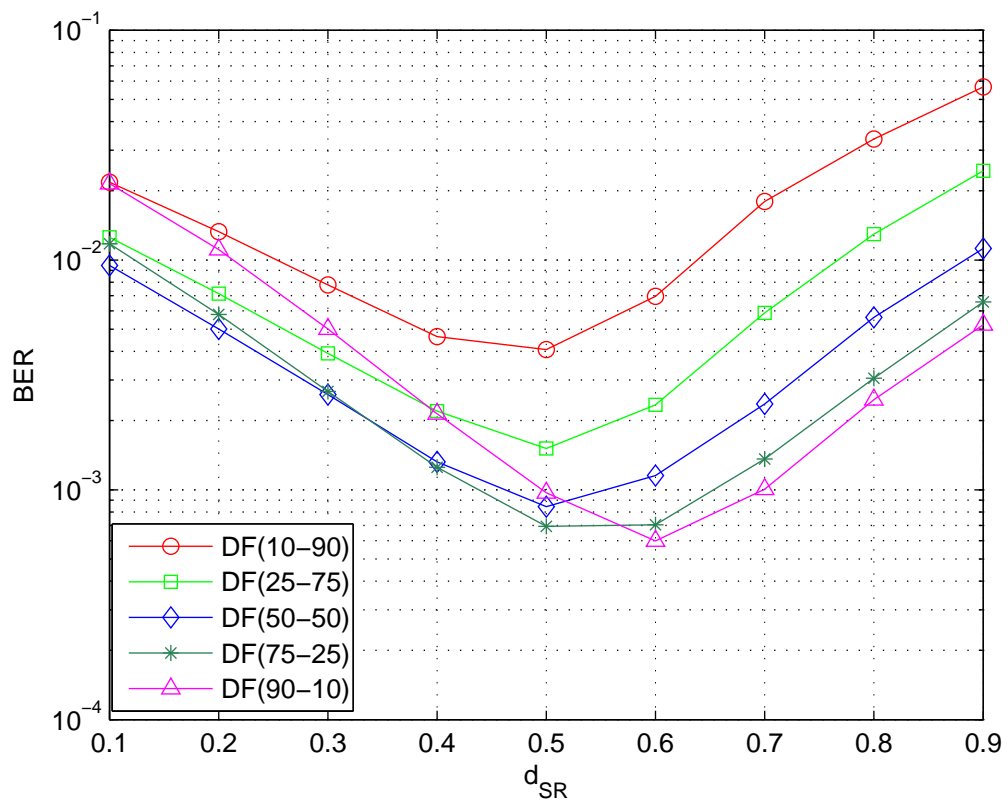

Figure 4.3: Comparison of semi-analytical BER performance of DF with various different power allocations in Phase 1 and Phase 2.

Fig. 4.3 and Fig. 4.4 shows how the performance varies with changing source to relay distance and also with different power allocations. Generally the best performance is achieved 


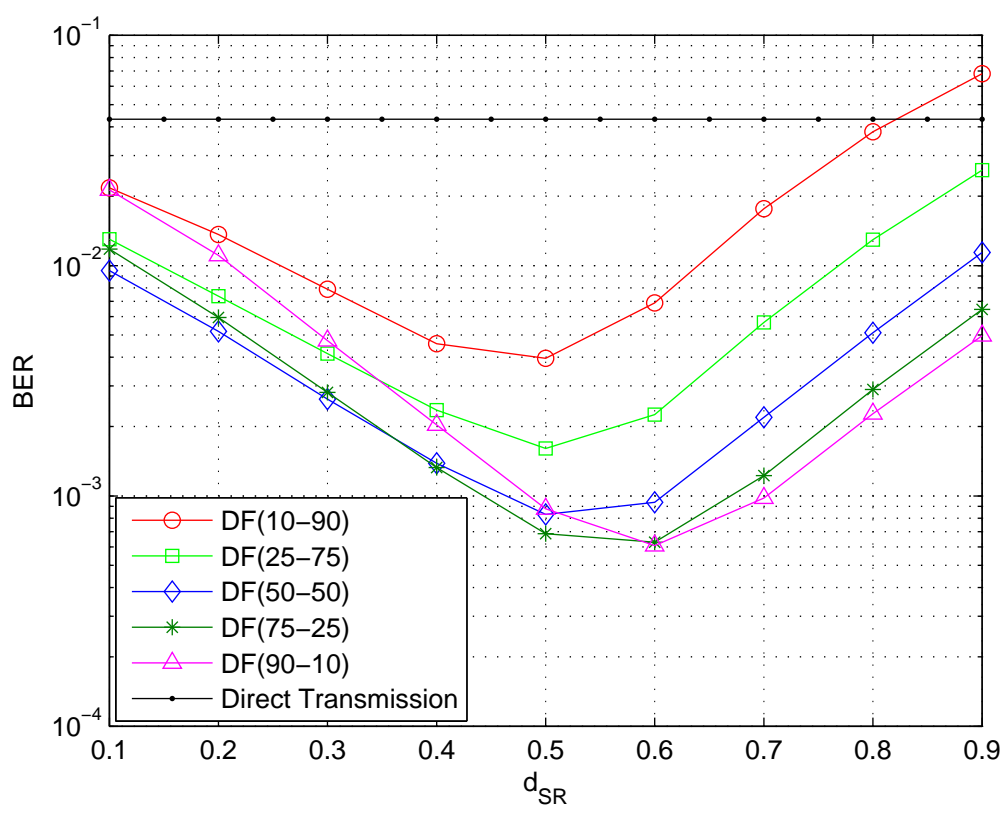

Figure 4.4: Comparison of simulated BER performance of DF with various different power allocations in Phase 1 and Phase 2 and direct communication.

around the midway region between source and destination and there is a particular power allocation that performs best at a particular location. Also the power allocation scheme in which 10 percent of the total transmit power is sent in Phase 1 and the rest in Phase 2, performs worse than the direct communication in areas near the destination. As the relay moves away from the source there is a general degradation of performance and the fact that only one tenth of the total transmit power is available in Phase 1 does not help. The signal decoded in this region would contain a large number of errors which in turn will lead to a lot of packets being discarded after CRC check at the relay thus leading to a severe degradation of performance.

So from Fig. 4.3 and Fig. 4.4 it can be seen that even for DF there should be some optimal power allocation corresponding to a particular location. We proceed to numerically optimize (4.5) for the Phase 1 power, $p_{1}$ using Quasi-Newton method [58, 59], under total power constraint given by (3.1).

With the help of this optimal power allocation we reevaluate the performance of our system and present the result in Fig. 4.5. As expected the optimal power allocation provides 
the best performance throughout the whole range. The gain over direct communication, when the relay is in between but collinear to the source and destination, is also worth noting. These incentives make power optimization a worthwhile exercise.

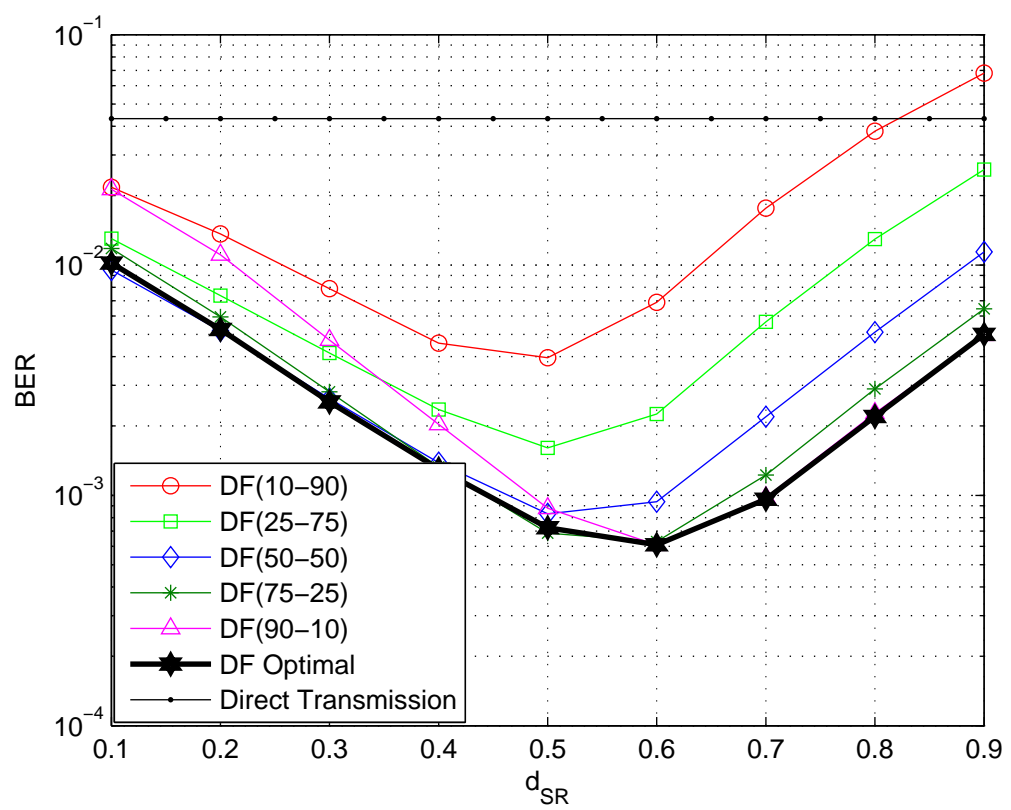

Figure 4.5: Comparison of simulated BER performance of DF with various different power allocations in Phase 1 and Phase 2, direct communication, and DF with optimal power allocation.

The effect of different SNRs on the performance of the power optimized system would give us an idea of how it responds to SNR changes. In Fig. 4.6 and Fig. 4.7 we evaluate the BER and throughput performance for $10 \mathrm{~dB}$ and $20 \mathrm{~dB}$ received SNRs and also compare them with those of direct communication. We can see from these figures that the system generally performs better at higher SNRs. It is also seen that the throughput of direct communication, with $20 \mathrm{~dB}$ received SNR, is much more closer to that of the power optimized case with same SNR, compared to the $10 \mathrm{~dB}$ received SNR case. This indicates to the fact that with arbitrarily large SNRs, the performance achieved with power optimized relaying and that of direct communication would ultimately coincide. 


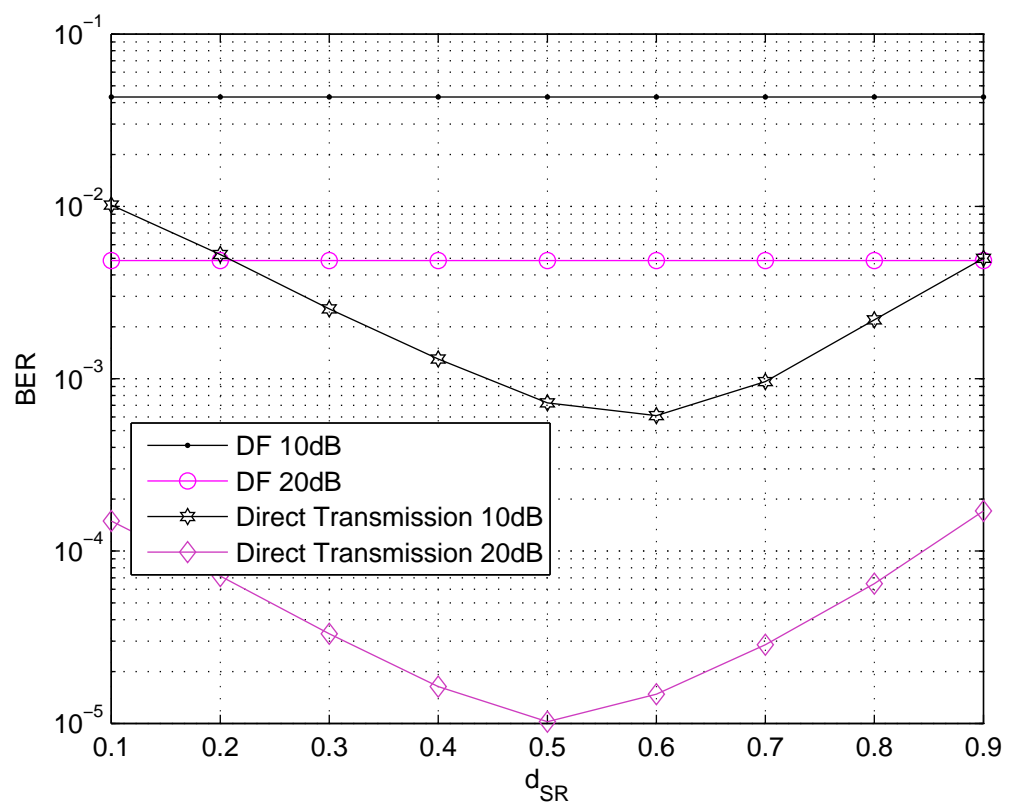

Figure 4.6: Comparison of simulated BER performance of DF with optimal power allocation and direct communication for received SNRs of $10 \mathrm{~dB}$ and $20 \mathrm{~dB}$.

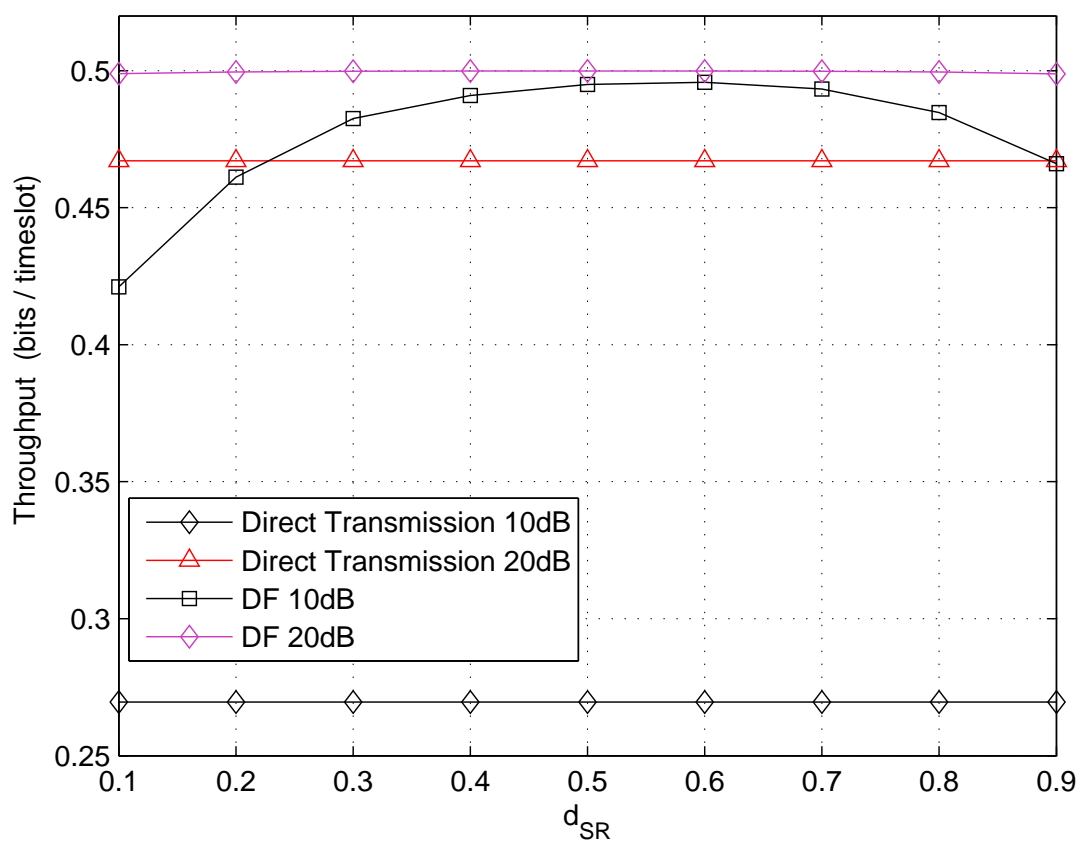

Figure 4.7: Comparison of the throughput of DF with optimal power allocation and direct communication for received SNRs of $10 \mathrm{~dB}$ and $20 \mathrm{~dB}$. 


\subsubsection{Power Optimization Over a Grid and Area of Interest}

We would now like to evaluate the system performance when the relay is free to move over a two dimensional grid in the same plane as the source and destination. A $3 \times 3$ grid, with the source at $\mathrm{x}=-0.5, \mathrm{y}=0$ and the destination at $\mathrm{x}=0.5, \mathrm{y}=0$ is chosen for the purpose. Fig. 3.9 in Chapter 3 shows this grid with the source and destination pointed out. The relay can move over this region but for computational purposes we assume the relay to be in any of the intersecting grid points. For each of these points there would be an optimum power allocation scheme. We optimize the power allocations for each grid point using QuasiNewton optimization on (4.4) and (4.6). The specific power allocations for each grid point thus obtained is shown in Fig.4.11 and taking into account these optimal power allocations we simulate the performance of the system over the grid and plot the BER performance in Fig. 4.8.

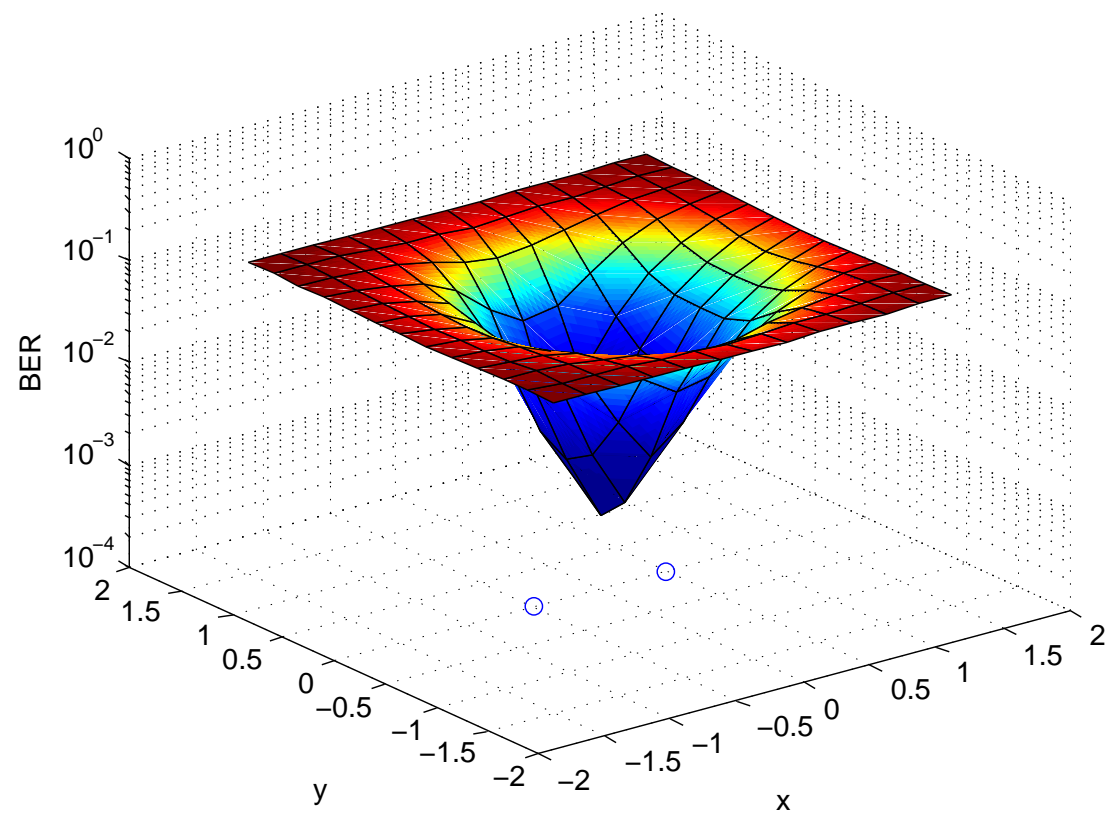

Figure 4.8: BER performance of DF over the grid. The received SNR is $10 \mathrm{~dB}$. Source at $\mathrm{x}, \mathrm{y}$ $=(-0.5,0)$ and destination at $\mathrm{x}, \mathrm{y}=(0.5,0)$.

It is again seen in Fig. 4.8 that the system achieves best performance around the midway region between the source and relay. Noting the fact that the system performance drops as the relay moves farther away from the source or destination, we might conjecture that there would be places in the grid where relaying using DF offers no better performance or even 
worse performance than direct communication. To prove this, we plot the performance of direct communication on the same plot as Fig. 4.8.

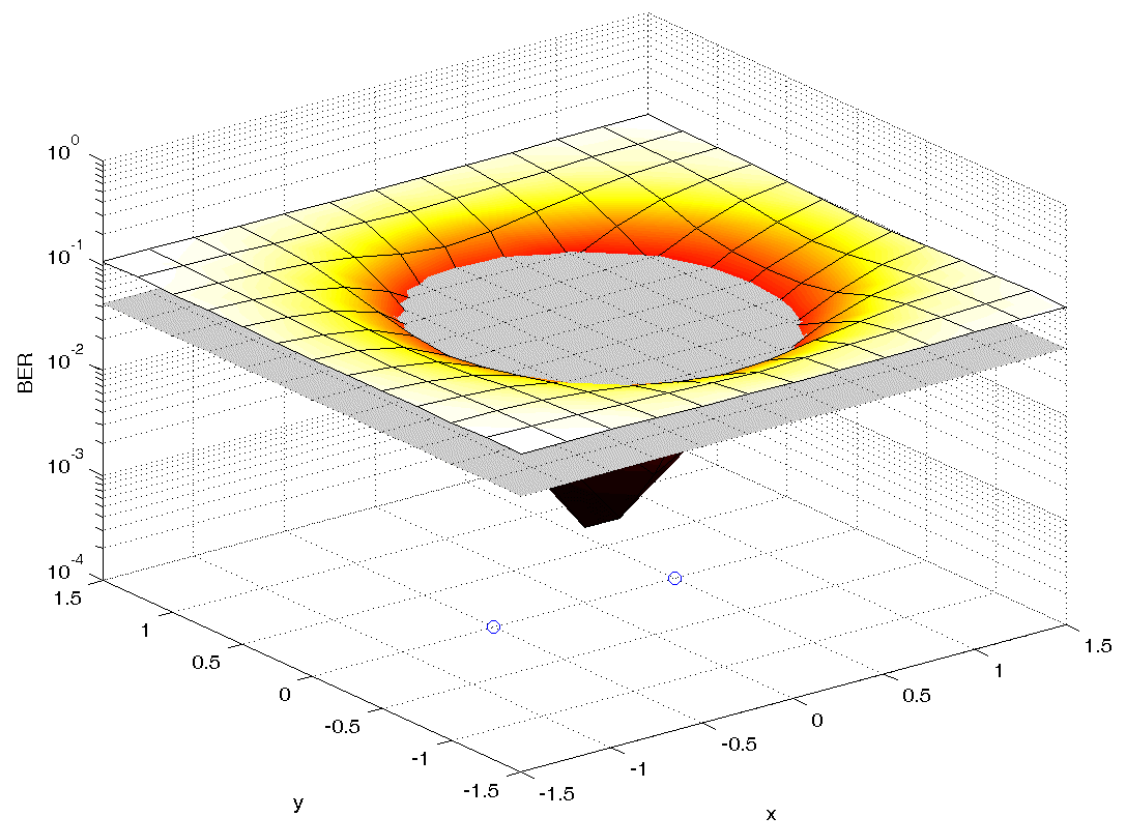

Figure 4.9: BER performances of DF over the grid and direct communication. The received SNR is $10 \mathrm{~dB}$. Source at $\mathrm{x}, \mathrm{y}=(-0.5,0)$ and destination at $\mathrm{x}, \mathrm{y}=(0.5,0)$.

The fact that DF can be worse is clearly seen in Fig. 4.9 with the region above the gray plane, representing the performance of the direct communication, representing areas where direct communication performs better. In Fig. 4.10 in order to map the regions where DF, with optimal power allocation, outperforms direct communication we look at Fig. 4.9 from the top.

Fig. 4.8 shows again that the best performance is achieved by positioning the relay about midway between the source and the relay provides the best BER performance. In Fig. 4.10 the central lighter, elliptical area depicts the region where relaying using decode and forward technique would give better performance over direct transmission. It is also noticed that not only is the shape of the region, where decode and forward is better than direct transmission, different than that for amplify and forward but it also has a greater area than the same for amplify and forward.

The portion of power used for transmission in Phase 1, $p_{1}$, is plotted in Fig. 4.11, which, 


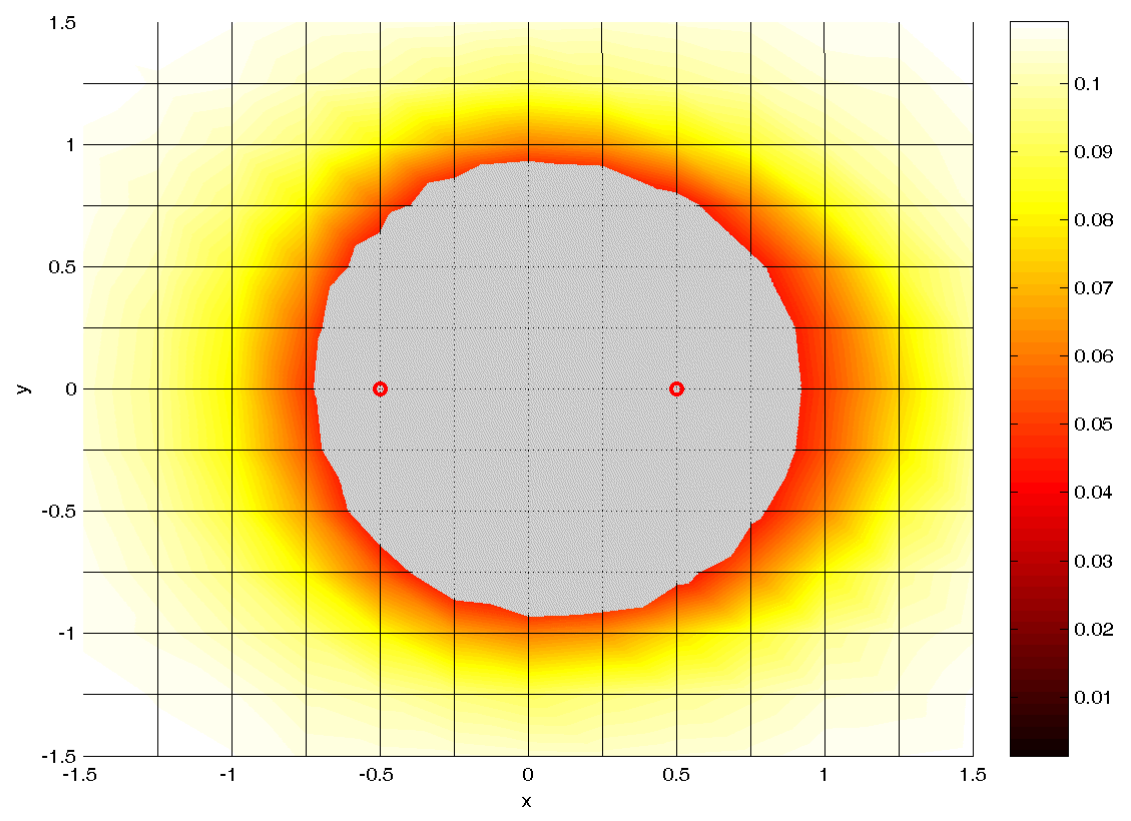

Figure 4.10: The inner lighter area represents the region for relaying in DF. The received $\mathrm{SNR}$ is $10 \mathrm{~dB}$. Source at $\mathrm{x}, \mathrm{y}=(-0.5,0)$ and destination at $\mathrm{x}, \mathrm{y}=(0.5,0)$.

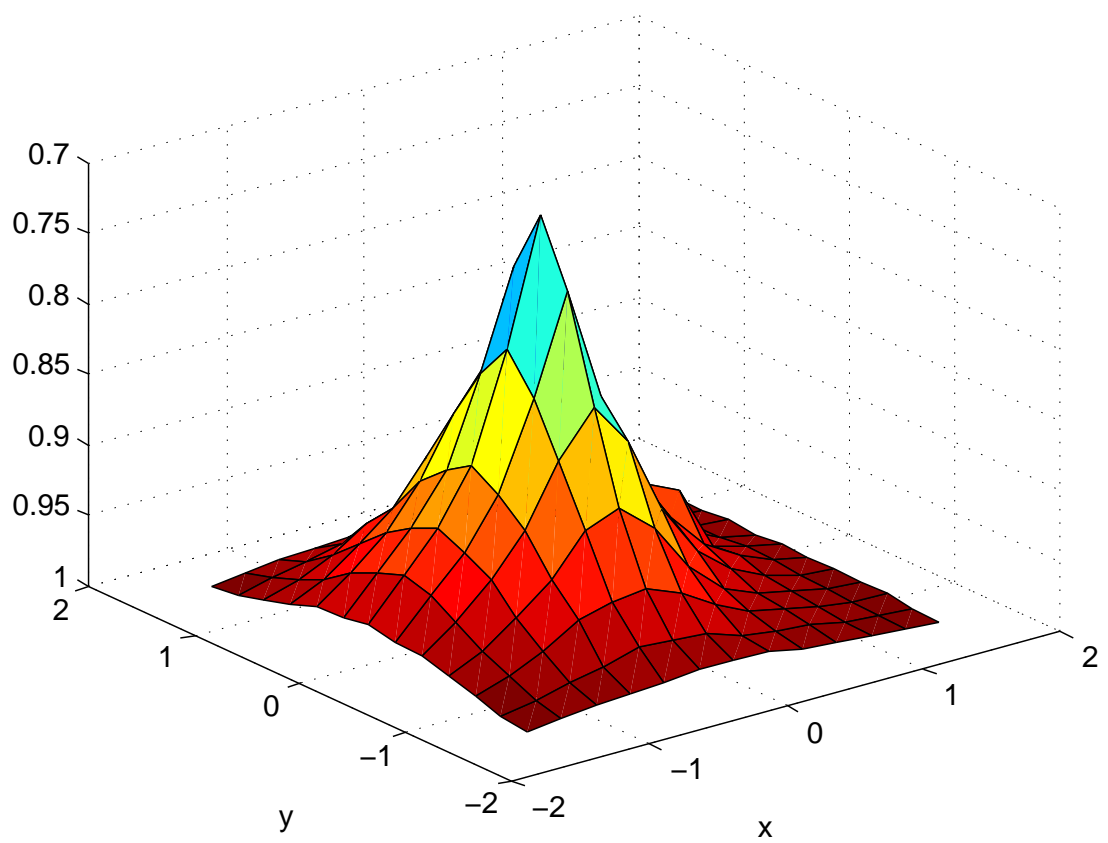

Figure 4.11: Phase 1 power $p_{1}$ for DF. The received SNR is $10 \mathrm{~dB}$. Source at $\mathrm{x}, \mathrm{y}=(-0.5,0)$ and destination at $\mathrm{x}, \mathrm{y}=(0.5,0)$. The $\mathrm{z}$ axis represents the optimal value of $p_{1}$.

as discussed in the amplify and forward section, implies that in the region where the system BER performance is better we can send comparatively lesser amount of power in Phase 1. 
We also note that the allocated power for AF and DF is very similar.

Fig. 4.12 shows the performances of the system for $10 \mathrm{~dB}$ and $20 \mathrm{~dB}$ received SNRs. We see that, predictably, at 20dB not only is the system performance better throughout but also over a wider area than that for lower SNRs.

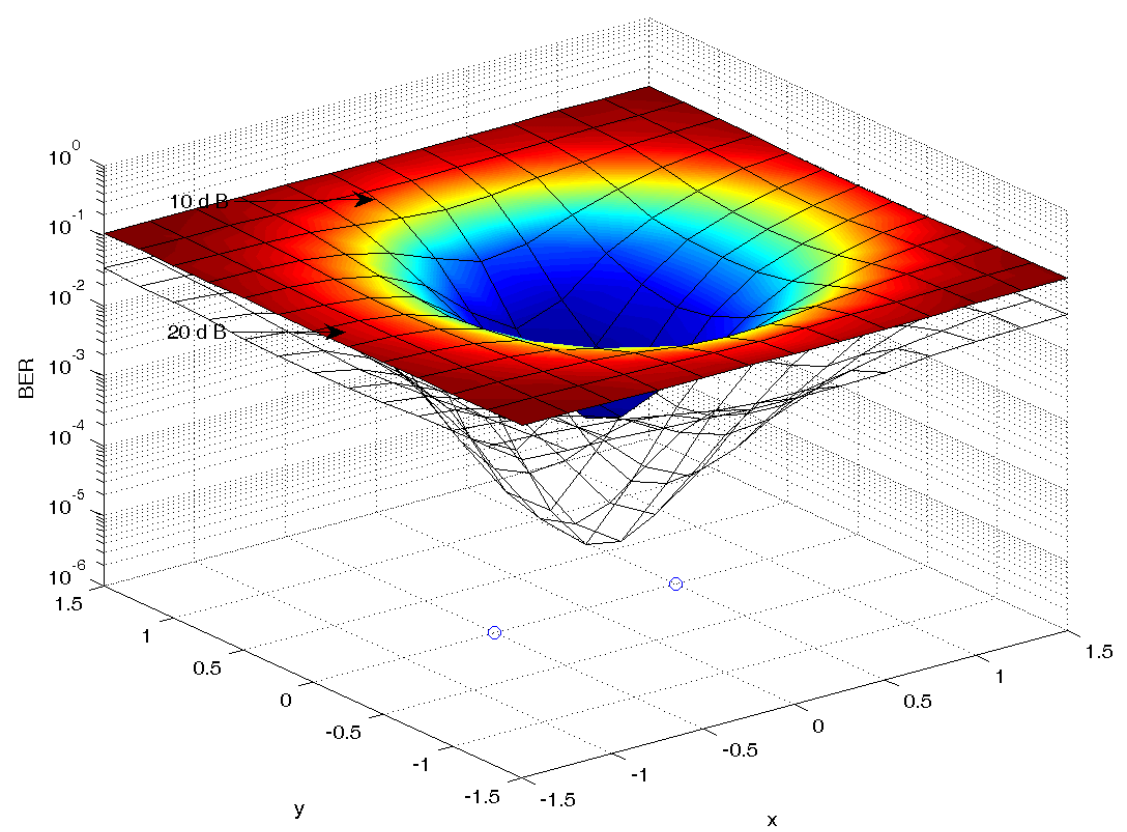

Figure 4.12: Comparison of BER performances of DF with received SNRs 10dB and 20dB. Source at $\mathrm{x}, \mathrm{y}=(-0.5,0)$ and destination at $\mathrm{x}, \mathrm{y}=(0.5,0)$.

As discussed in Chapter 3, we would like to know how the area of interest, where relaying is relevant changes with changes in received SNR. To check this we simulate the performance of the power optimized system and that of direct communication, for $10 \mathrm{~dB}$ and $20 \mathrm{~dB}$ received SNR, and compare the region where relaying provides performance gains. In Fig. 4.13 it can be clearly seen that for $20 \mathrm{~dB}$ received SNR there is a significant increase in the size of the area of interest. This suggests that the relative amount by which the performance of the relay communication improves over the area in question is more than that for direct communication. 

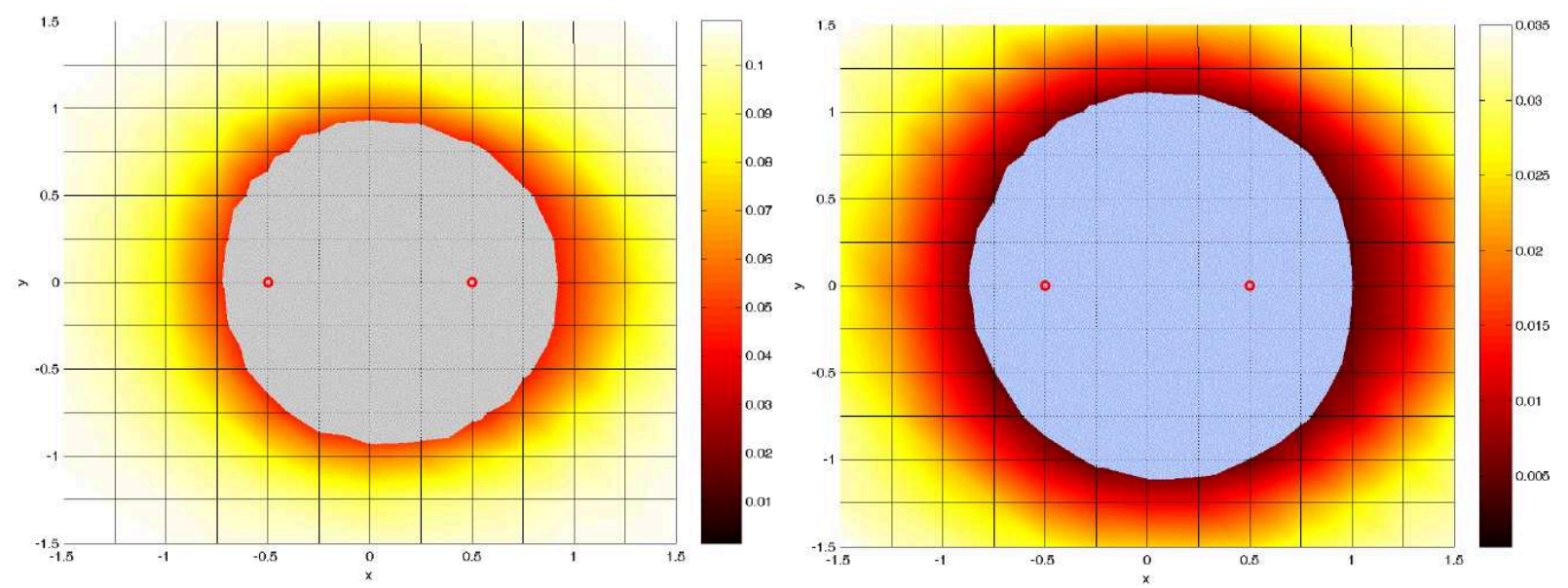

Figure 4.13: The inner lighter areas represent the region for relaying in coherent environment using DF at the relay. The received SNR for the left hand plot is $10 \mathrm{~dB}$ and that for the right hand one is $20 \mathrm{~dB}$. Source at $\mathrm{x}, \mathrm{y}=(-0.5,0)$ and destination at $\mathrm{x}, \mathrm{y}=(0.5,0)$.

\subsection{Performance Comparison of Amplify Forward and Decode Forward}

Having established the basic framework we now proceed to analyze our results. We consider various arbitrary power allocations for both $\mathrm{AF}$ and $\mathrm{DF}$ and evaluate their performance assuming that the relay is allowed to be in any position along a straight line between the source and the destination which are unit distance apart. From Fig. 4.14 and Fig. 4.15, we see that using the power optimization technique we get the best overall performance, reiterating the importance of optimal power allocation.

In Fig. 4.16 we look at the performances of AF, DF and direct transmission over a grid. We notice that the DF protocol, wherein the relay transmits only when it decodes correctly, outperform the non-regenerative AF protocol, which forwards all the time. Not only is the performance of DF better than AF, it also has wider region where relaying is meaningful. This is also evident from Fig. 3.13 and Fig. 4.10. The throughput of DF is also better than that of the AF as shown in Fig. 4.17, which shows the throughputs of AF and DF using optimal power allocation when the relay is collinear with the source and destination. By throughput we will mean throughput per timeslot in this thesis. Considering that we have a half duplex system, we get better throughput and BER for both AF and DF when the relay 


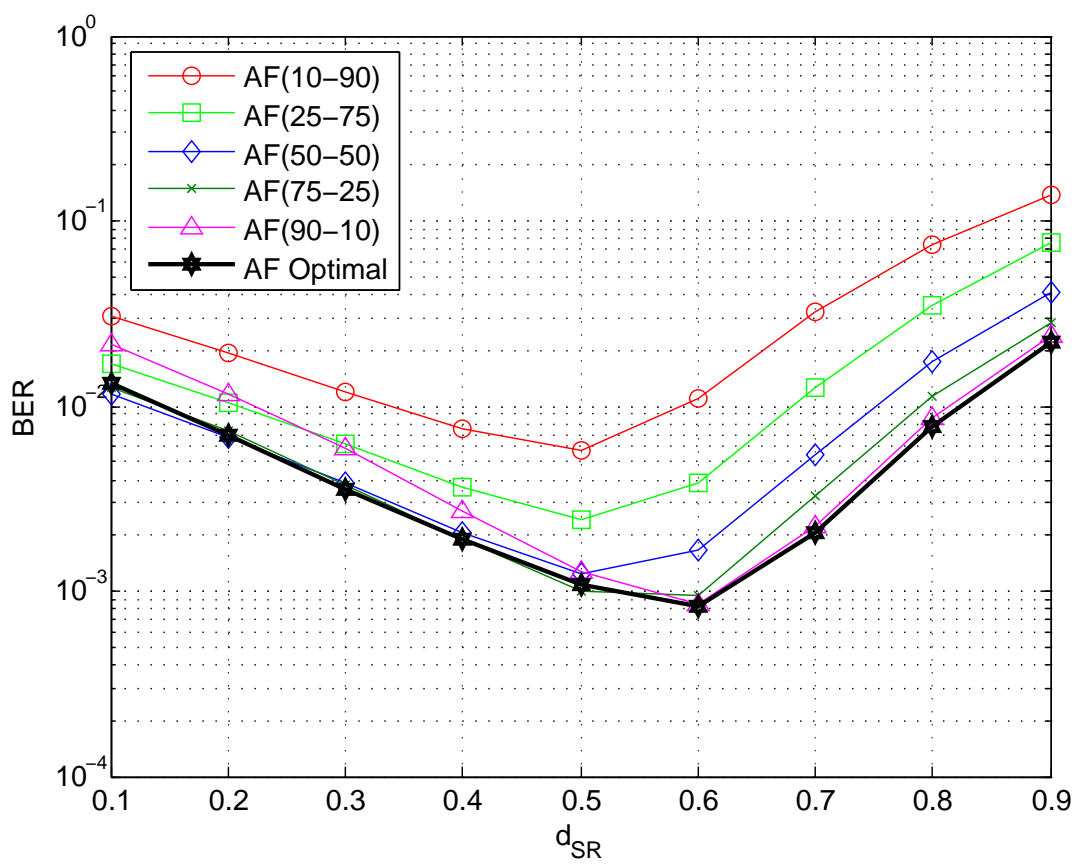

Figure 4.14: BER performance of AF with arbitrary and optimal power allocation. The received $\mathrm{SNR}$ is $10 \mathrm{~dB}$. Source at $\mathrm{x}, \mathrm{y}=(0,0)$ and destination at $\mathrm{x}, \mathrm{y}=(1,0) . \mathrm{AF}(10-90)$ represents the case when 10 percent of the total power is sent in Phase 1 and 90 percent in Phase 2 and so on.

is in the midway region. From Fig. 4.17 it is also seen that by using our optimized power allocation there are throughput gains over the method used in [37].

In [37] the authors have proposed a power allocation scheme from an information theoretic angle with the aim of maximizing the channel capacity and SNR in a relaying system using regenerative DF protocol. There the transmitter knows the instantaneous channel state information, which allows it to switch the relay off when the relay to destination link instantaneous SNR $\left(a_{2}\right)$, is as good or worse than the direct link instantaneous SNR $\left(a_{0}\right)$ or when the direct link instantaneous SNR is better than the source to relay link instantaneous SNR $\left(a_{1}\right)$ but worse than the relay to destination link instantaneous SNR. Otherwise, when the direct link is worse or as good as the source to relay link and also worse than the relay to destination link, according to [37], the power $p_{1}$ in Phase 1 is given by

$$
p_{1}=\frac{a_{2}}{a_{1}+a_{2}-a_{0}} \times \mathcal{P}_{\text {Total }}
$$




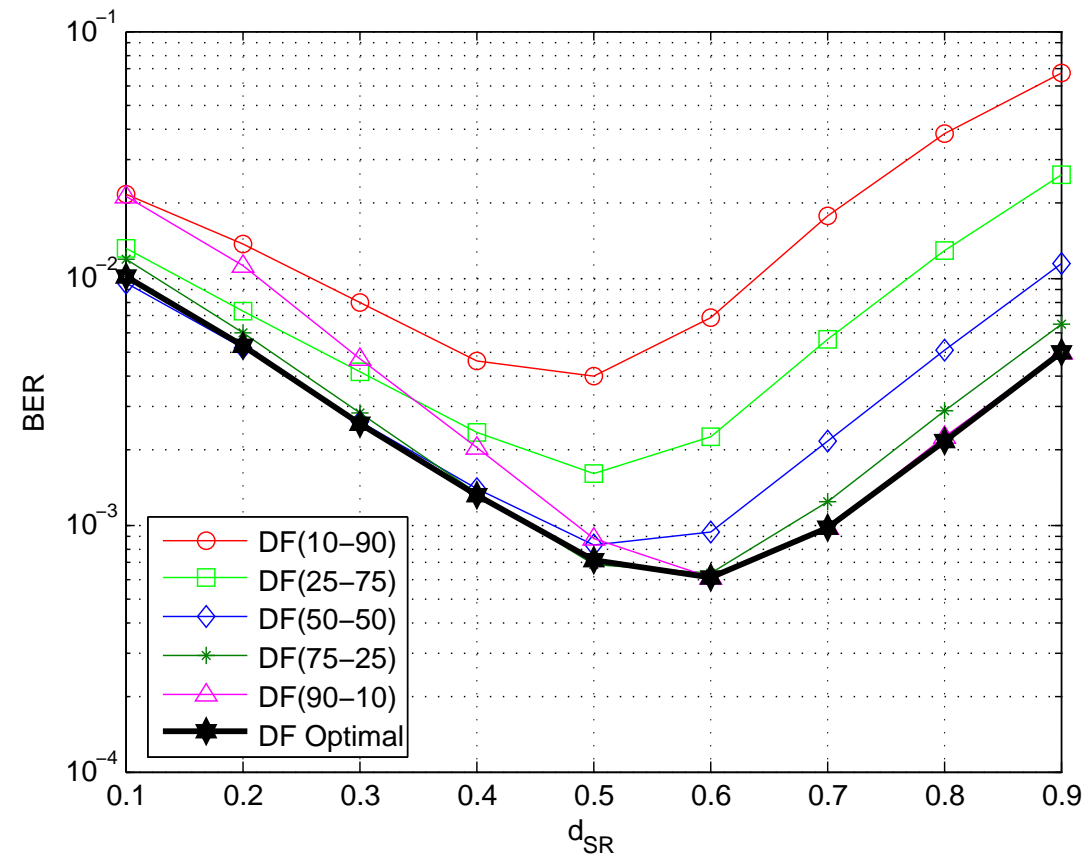

Figure 4.15: BER performance of DF with arbitrary and optimal power allocation. The received $\mathrm{SNR}$ is $10 \mathrm{~dB}$. Source at $\mathrm{x}, \mathrm{y}=(0,0)$ and destination at $\mathrm{x}, \mathrm{y}=(1,0)$. $\mathrm{DF}(10-90)$ represents the case when 10 percent of the total power is sent in Phase 1 and 90 percent in Phase 2 and so on.

From Fig. 4.18 we can see that our DF protocol, with optimized power, matches or outperforms theirs at most places, even though the relay in our system is never fully switched off. When we use the power allocation in [37] we see that in areas where $a_{1} \geq a_{0}$ and $a_{2}>a_{0}$, $p_{1}$ in (4.7) becomes a very small portion of the total power and hence the loss in throughput and degeneration of BER in those regions. This can be clearly seen in Fig. 4.17 and Fig. 4.18 between $d_{s, r}=0.15$ and $d_{s, r}=0.5$.

Lastly, we compare the throughput performance of AF and DF, over the grid we considered, in Fig. 4.19 and notice that the throughput for DF is better than AF at most places and even at places where it is worse, it is only by a very small amount.

\subsection{Conclusion}

Position dependent optimized power allocation in cooperative relay networks provide performance enhancement irrespective of the forwarding protocol used at the relay and over 


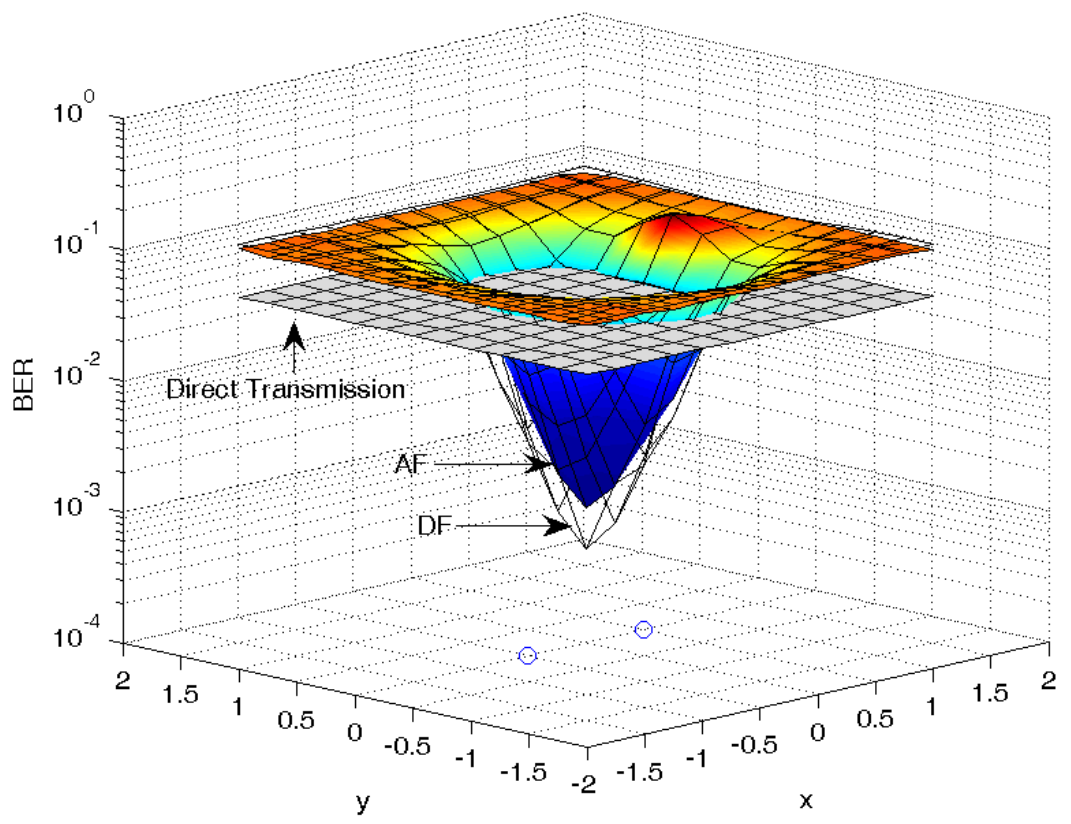

Figure 4.16: BER performance of $\mathrm{AF}$ and $\mathrm{DF}$ with optimal power allocation and direct transmission. The received SNR is $10 \mathrm{~dB}$. Source at $\mathrm{x}, \mathrm{y}=(-0.5,0)$ and destination at $\mathrm{x}, \mathrm{y}=$ $(0.5,0)$.

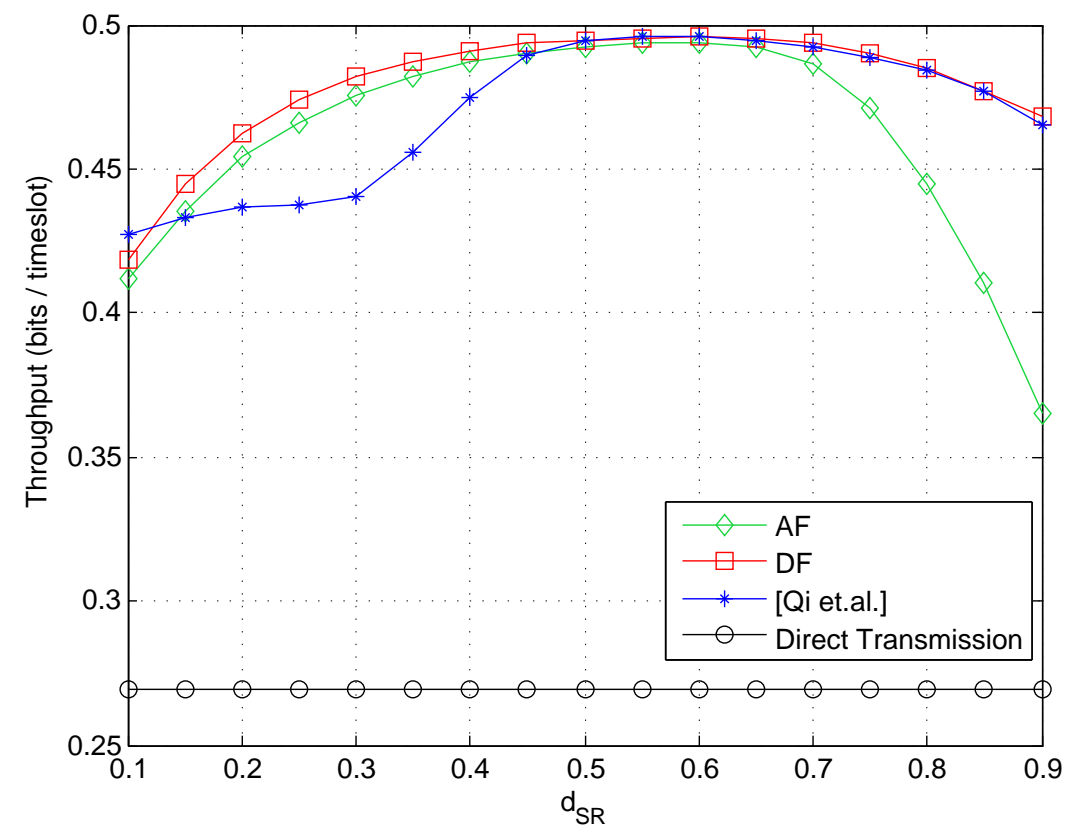

Figure 4.17: Comparison of throughput performance between [Qi et.al.] and our model for DF and AF with optimal power allocation. The received SNR is 10dB. Source at $\mathrm{x}, \mathrm{y}=(0,0)$ and destination at $\mathrm{x}, \mathrm{y}=(1,0)$. 


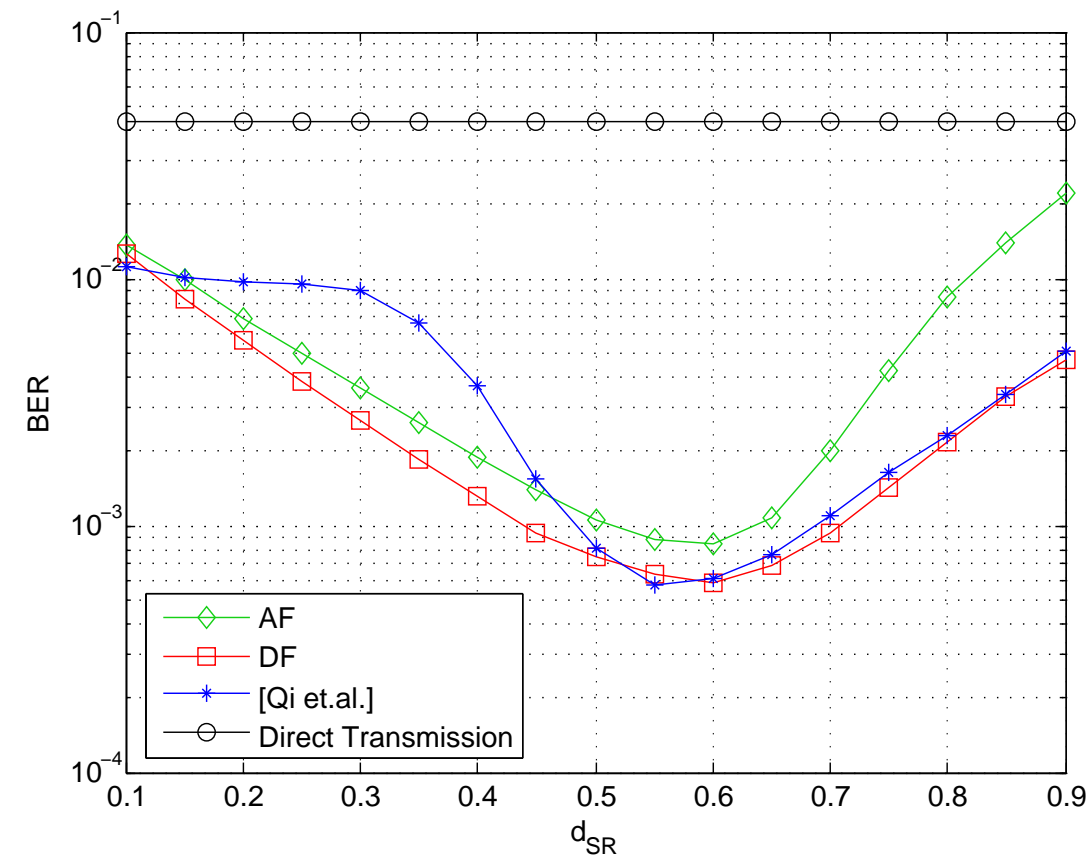

Figure 4.18: Comparison of BER performance between [Qi et.al.] and our model for DF. The received SNR is $10 \mathrm{~dB}$. Source at $\mathrm{x}, \mathrm{y}=(0,0)$ and destination at $\mathrm{x}, \mathrm{y}=(1,0)$.

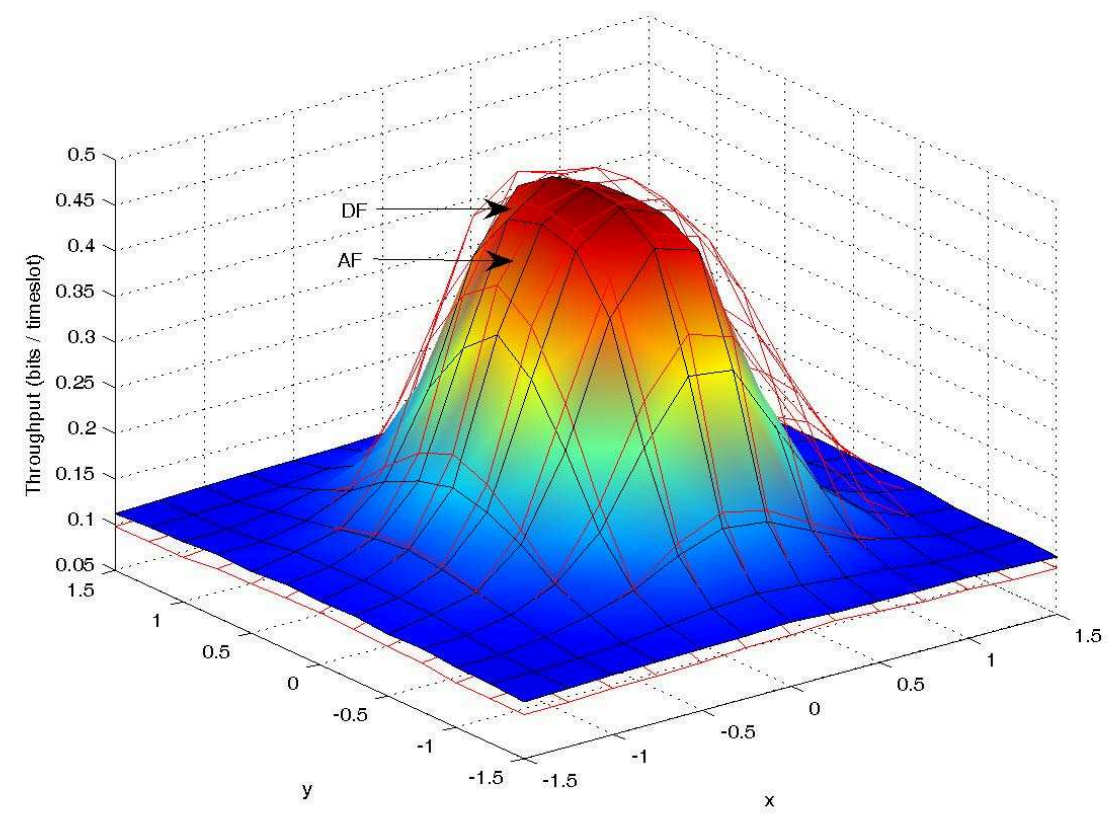

Figure 4.19: Comparison of throughput performances of AF and DF using our power optimized model. The received SNR is $10 \mathrm{~dB}$. Source at $\mathrm{x}, \mathrm{y}=(-0.5,0)$ and destination at $\mathrm{x}, \mathrm{y}=$ $(0.5,0)$. 
nonoptimal power allocation schemes. Some of the nonoptimal schemes work differently in $\mathrm{AF}$ and DF at certain areas, but the optimal power allocation is very similar for both the protocols, pointing to the fact that there are certain powers which perform best in both AF and DF for a particular area. This, with further studies, might lead into a better understanding about the effect and importance of power allocation in cooperative relay networks. We identify the cardioid and elliptical shaped regions for AF and DF where relaying would provide better performance than direct transmission and show that this area increases with increasing SNR for both the forwarding protocols. It is also noticed that this region is greater for DF than AF i.e. for the same received SNR, DF has a wider area around the source and destination where relaying provides performance benefits than AF. Also in this region DF outperforms AF. We conclude that, though we can place the relay anywhere in our grid and use any forwarding technique, it makes practical sense to use DF and place the relay around the midway region between the source and destination, which in turn lies inside the region where relaying is relevant, because around that region our optimal power allocation scheme can extract the best performance of the system. 


\section{Chapter 5}

\section{Forwarding with Noncoherent Modulation}

\subsection{Introduction}

In a real world scenario like a fast fading environment the transmitter or receiver may not have the luxury of knowing the channel state information (CSI). Realistic wireless signals are prone to undergo various kinds of fading and time delay which sometimes make it difficult to accurately estimate the channel state information. In additive Gaussian noise environments coherent demodulation has a $3 \mathrm{~dB}$ performance gain over noncoherent demodulation, even though the receiver structure for the former is more complex. So noncoherent demodulation, with a simpler receiver structure, can be a boon in practice, as it requires no CSI and also performs well in fast frequency selective fading channels with considerable phase noise, large doppler spread and co-channel interference. We will use frequency shift keying, specifically 4-FSK, with a noncoherent receiver structure as described in Fig. 5.1] [31], which is another way of representing $M$-FSK other than the one described in Chapter 2.

For our case the number of symbols used, $M=4$, since we are using 4-FSK. Here a decision can be arrived at by detecting the energy of the signal at each frequency and then checking which one of the $M$ branches has the highest energy amongst them. The receiver then outputs that $i^{\text {th }}$ message, $m_{i}$ as the decision. So we see that there is no coherent phase dependence in this receiver. If we transmit the $i^{\text {th }}$ symbol, the corresponding transmitted 


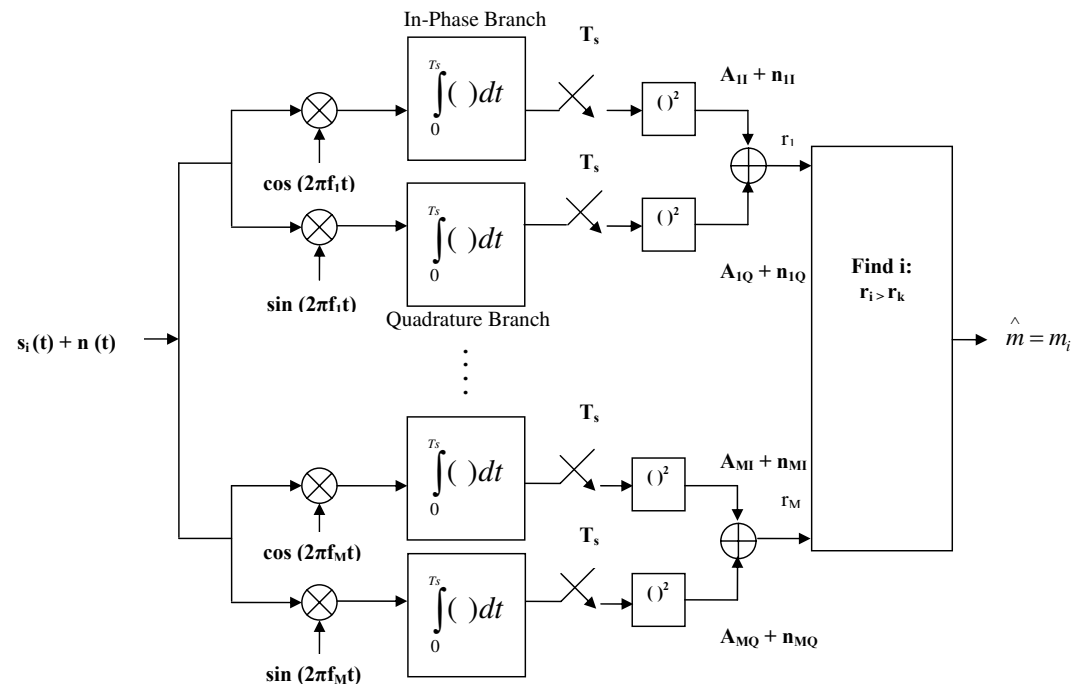

Figure 5.1: Receiver structure for M-FSK with noncoherent modulation.

signal would be

$$
s(t)=A \cos \left(2 \pi f_{i} t+\phi\right)=A \cos (\phi) \cos \left(2 \pi f_{i} t\right)-A \sin (\phi) \sin \left(2 \pi f_{i} t\right), 0 \leq t<T_{s},
$$

where $T_{s}$ is the symbol period and $\phi$ the phase offset between the transmitter and receiver oscillators at frequency $f_{i}$. In case the phase offset, $\phi=\frac{\pi}{2}$, the first term $A \cos (\phi) \cos \left(2 \pi f_{i} t\right)$ of the received signal can be almost zero. A coherent receiver which only detects the first term can, in such scenarios produce erratic results. In order to fix this problem the receiver in Fig. 5.1 splits the received signal into $M$ branches corresponding to each frequency $f_{k}$, $k=1, \ldots, M$. The received signal is multiplied by a noncoherent in-phase and quadrature carrier for each carrier frequency $f_{k}, k=1, \ldots, M$ and then integrated over a symbol time, sampled and squared. The output of the squarer of the $k^{\text {th }}$ branch for the in-phase and quadrature component is denoted by $A_{k I}+n_{k I}$ and $A_{k Q}+n_{k Q}$ correspondingly, where $n_{k I}$ and $n_{k Q}$ are effects of the receiver input noise $\mathrm{n}(\mathrm{t})$. Now for $i=k$ we get, $A_{k I}=A^{2} \cos ^{2}(\phi)$ and $A_{k Q}=A^{2} \sin ^{2}(\phi)$ and for $i \neq k$ we get, $A_{k I}=A_{k Q}=0$. It can now be clearly seen that the input to the decision device of the $i^{\text {th }}$ branch is going to be $A^{2} \cos ^{2}(\phi)+A^{2} \sin ^{2}(\phi)=A^{2}$ in the absence of noise, which is independent of $\phi$, and all the other branches will have zero input. So for every symbol period the branch with the largest energy is chosen and the corresponding symbol is chosen as the output. 
Using our system model described in Chapter 3 and 4-FSK instead of QPSK, we move on to investigate the effects of $\mathrm{AF}$ and $\mathrm{DF}$ in a noncoherent scenario.

\subsection{Performance Improvement Offered by Amplify and Forward and Decode and Forward}

In this section we would see that even with noncoherent modulation there could be performance benefits over direct communication when using intermediate relays between the source and the destination. We used both AF and DF at the relay and optimized the power allocation in Phase 1 and Phase 2 depending on the relay position. In both cases our scheme performed better than direct communication.

\subsubsection{Amplify and Forward:Collinear Case}

We will start with a scenario when the relay is allowed to move collinearly between the source and destination. Since in this case we assume no CSI, the performance may not be as good as with coherent demodulation. But it would be our aim to extract the best performance from the system, with the usual constraint of transmit power.

Using AF at the relay we evaluate the performance of the system for a number of different power allocations. This is shown in Fig. 5.2. At the relay, the signal with the highest energy is chosen and transmitted towards the destination. The destination also chooses the signal with the highest energy from the transmission it receives directly from the source. The destination finally chooses the highest energy signal from both the signals it receives from the relay and source and add them up before making decision on the received data. The received $\mathrm{SNR}$ is $10 \mathrm{~dB}$ for all the cases. We also plot the best BER envelope,

$$
\operatorname{BER}_{\text {min }}(d)=\min _{p_{1}, p_{2}} \operatorname{BER}\left(p_{1}, p_{2}, d\right),
$$

by picking the best BER at each relay position, to get a glimpse of the system performance with optimal power allocation.

As seen in the previous chapters, it is again noticed that different power allocations behave differently at different regions. We plot the performance of the direct communication also 


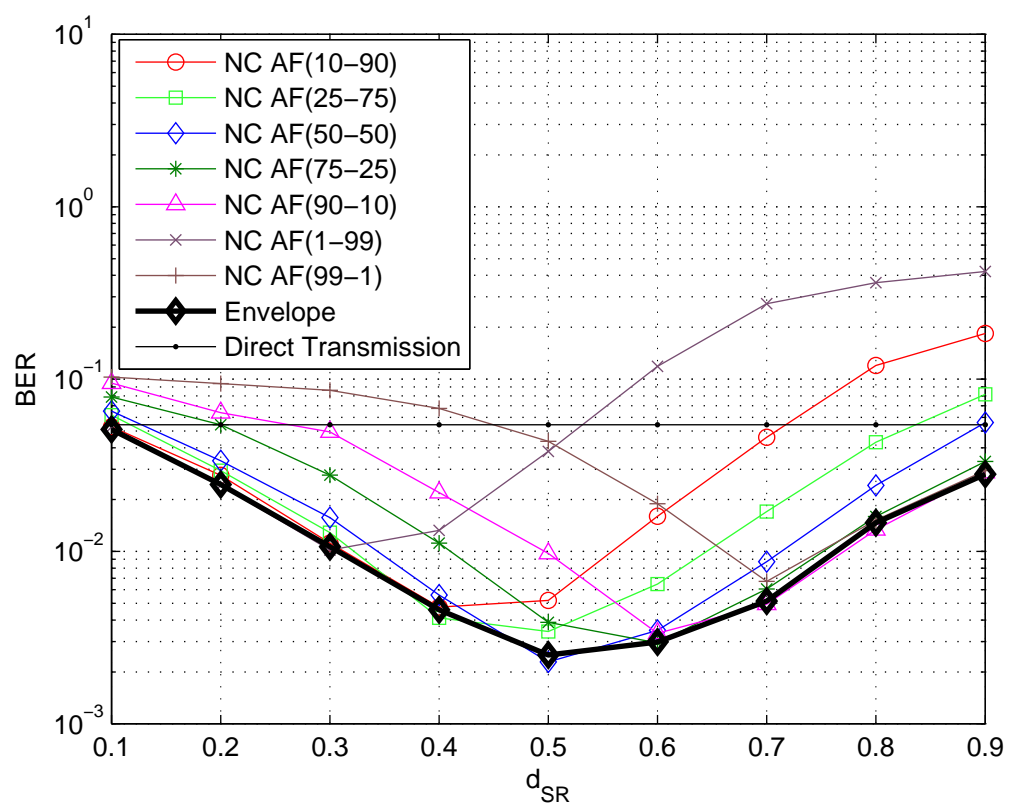

Figure 5.2: Comparison of simulated BER performance of AF with various different power allocations in Phase 1 and Phase 2 and direct communication. The envelope represents the best $\mathrm{BER}$ performance given by $\min _{p_{1}, p_{2}} \operatorname{BER}\left(p_{1}, p_{2}, d\right)$.

and see that at certain places it works better than some power allocations. But the fact that there could be a position dependant transmission power allocation, indicated by the envelope, is also recognized. But before we exploit this potential for performance enhancement, we will evaluate the behavior of DF in the same setting.

\subsubsection{Decode and Forward:Collinear Case}

The forwarding technique used here is, DF, as explained in detail in Chapter 4. In this case, where we use noncoherent modulation, we will see how it performs under different power allocations assuming total transmit power constraint. The signals are combined at the destination in the same way as in noncoherent AF. Fig. 5.3 shows the performance of different power allocations and that of direct transmission for $10 \mathrm{~dB}$ received SNR.

The performances appear to be very similar to those of AF so we proceed to compare the BER curves of AF and DF in Fig. 5.4. The dashed lines represent results for AF and the solid ones that for DF. We see that, indeed the performance of both AF and DF are 


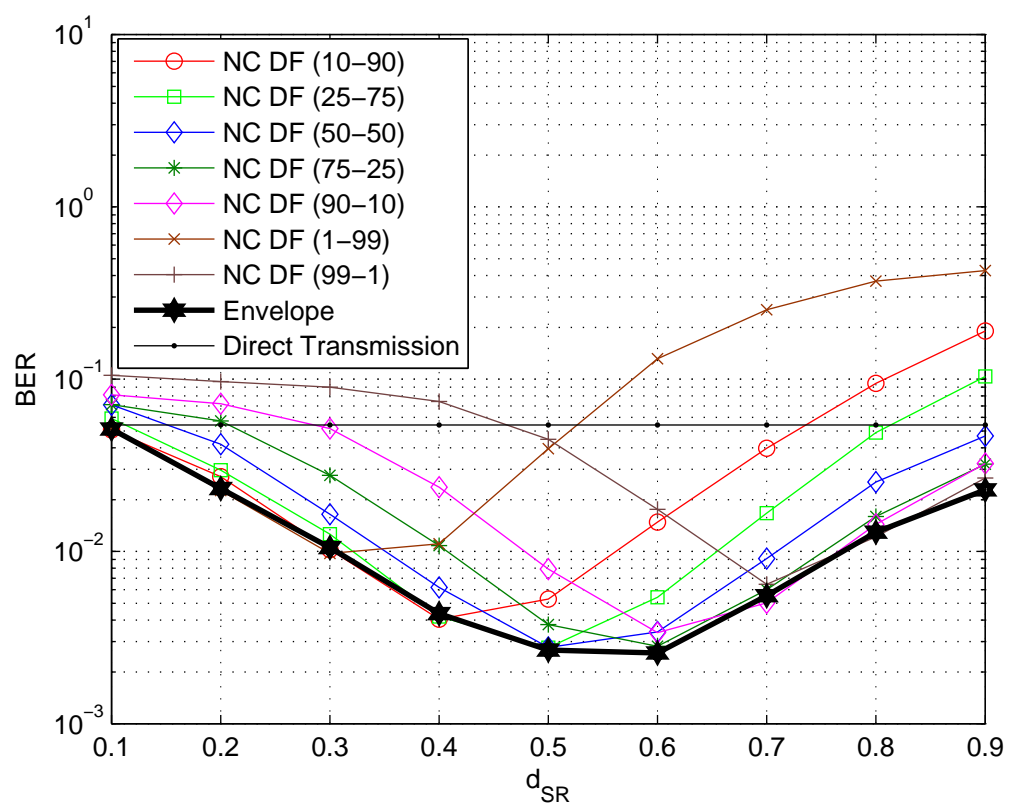

Figure 5.3: Comparison of simulated BER performance of DF with various different power allocations in Phase 1 and Phase 2 and direct communication. The envelope represents the best BER performance given by $\min _{p_{1}, p_{2}} \operatorname{BER}\left(p_{1}, p_{2}, d\right)$.

almost identical when noncoherent modulation is used. We should reiterate here that in our DF protocol the relay forwards only correctly received and decoded data.

\subsection{Power Optimization in Collinear Case}

In Chapter 4 we have discussed in detail the power optimization proposed by Qi et.al. in [37]. We adapt it to our model and now proceed to check its performance in the noncoherent scenario. We see from the Fig. 5.5 that Qi's power allocation scheme follows the best performance envelope closely, except very near the destination. In their scheme CSI at transmitter is assumed and they reserve no power for the relay if the direct link is better or as good as the relay to destination link or if the direct link is better than the source to relay link but worse than the relay to destination link. In our case the destinations always listens to the relay-destination channel and does not know if the relay is not allotted power for transmission in Phase 2. It might make practical sense to reserve even a very small amount of power $\left(\approx 10^{-5} W\right)$ for the relay to transmit in Phase 2 and with that diversity 


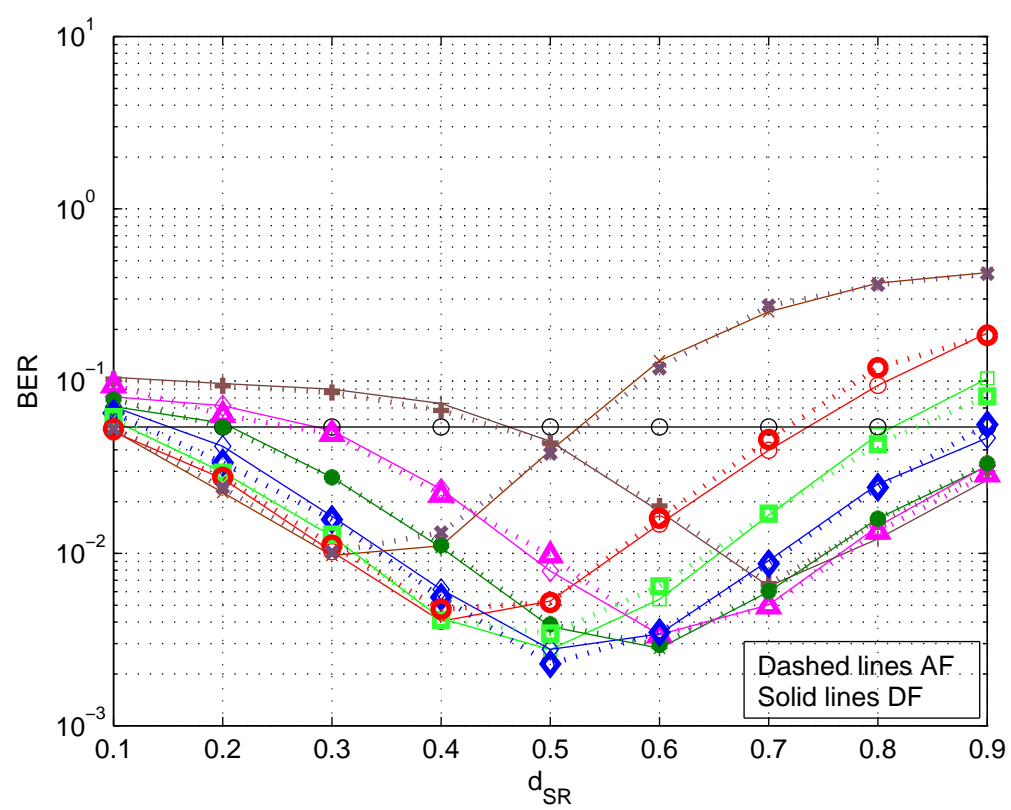

Figure 5.4: Comparison of simulated BER performance of AF and DF with various different power allocations in Phase 1 and Phase 2. The straight line represents direct communication.

the system performance in those areas should improve. Keeping this in mind we modify Qi's power allocation and reserve a very small amount of power for use by the relay in Phase 2 . We reevaluate the system performance and notice in Fig. 5.6 that the assumption was right and there is actual improvement of performance in the region near the destination. The new performance curve also matches the best performance envelope.

Since in noncoherent modulation using our model the performance of AF and DF is almost identical, we follow the same line for DF and and arrive at the modified power allocation scheme. First we check Qi's power allocation [37] and see in Fig. 5.7, that the same problem of bad performance near the destination, as seen in AF, is present here too. Arguing as before in AF, we modify Qi's scheme and allow the relays to transmit with a very small amount of power, instead reserving no power for them. Using this scheme, the performance of DF in the collinear case is simulated and the result shown in Fig. 5.7.

It is again seen that the modified power allocation scheme and the best performance envelope match closely. So the fact that reserving even a small amount of power for the relay to transmit in Phase 2 provides gain in terms of system performance, is reconfirmed. But it might happen that even with this modified scheme, the system performance becomes worse 


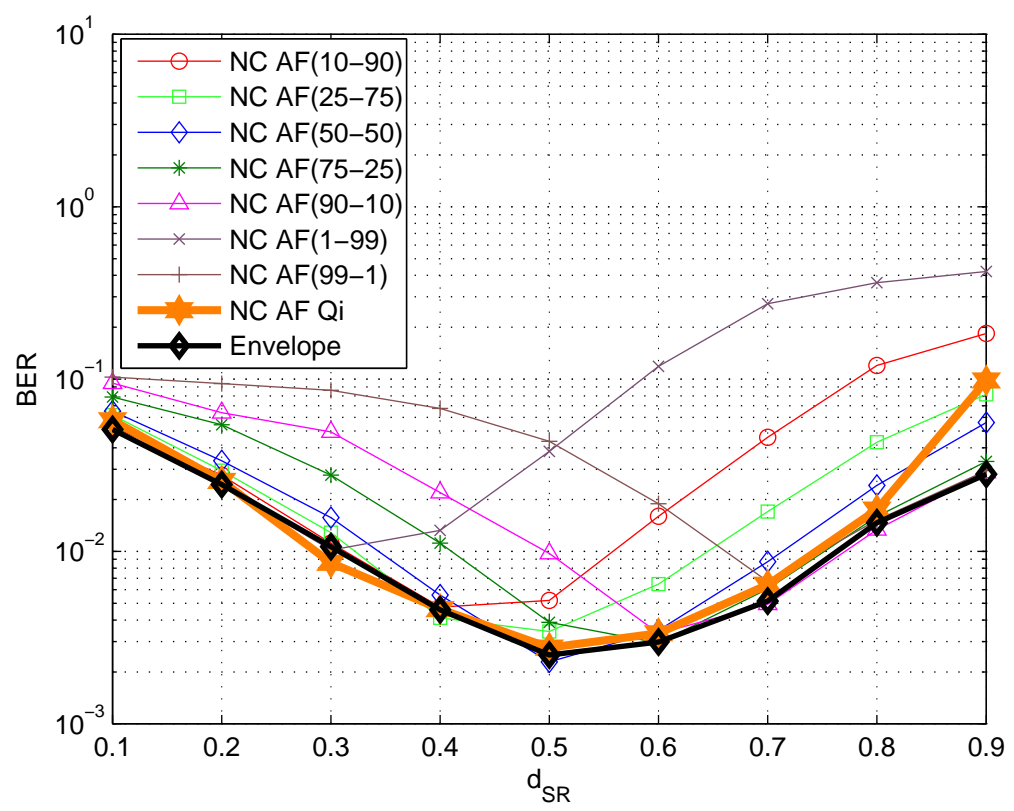

Figure 5.5: Comparison of simulated BER performance of AF with various different power allocations in Phase 1 and Phase 2 and Qi's power allocation scheme. The envelope represents the best BER performance given by $\min _{p_{1}, p_{2}} \operatorname{BER}\left(p_{1}, p_{2}, d\right)$.

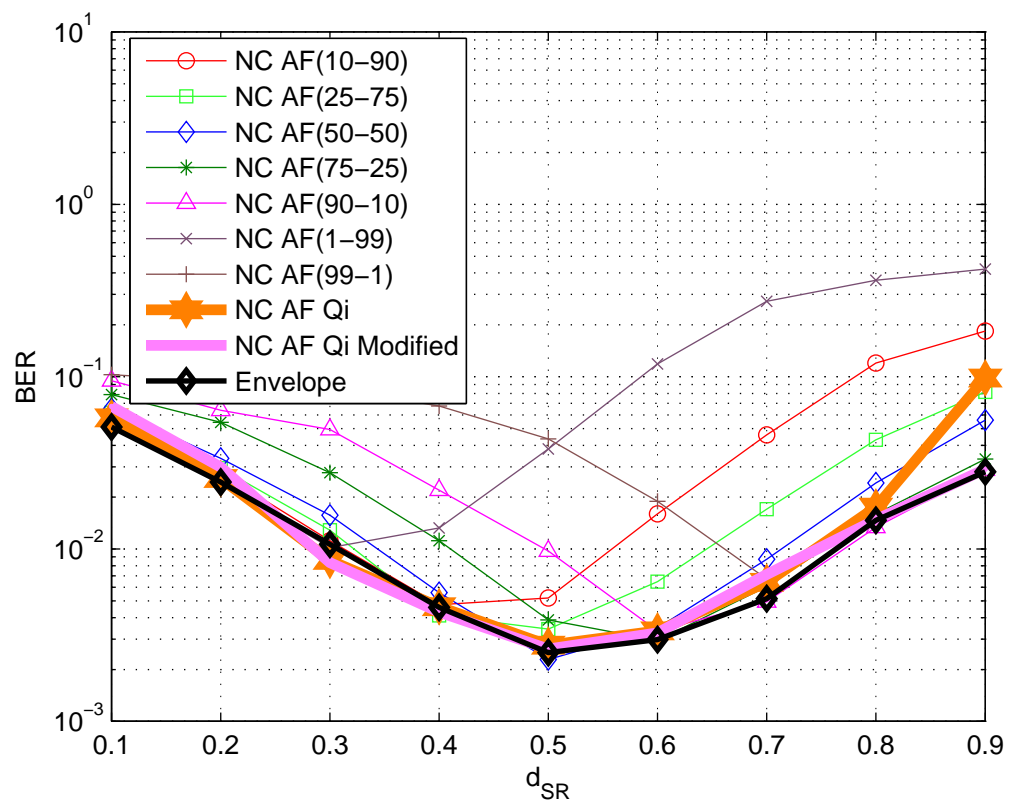

Figure 5.6: Comparison of simulated BER performance of AF with various different power allocations in Phase 1 and Phase 2 and Qi's original and modified power allocation scheme. The envelope represents the best $\operatorname{BER}$ performance given by $\min _{p_{1}, p_{2}} \operatorname{BER}\left(p_{1}, p_{2}, d\right)$. 


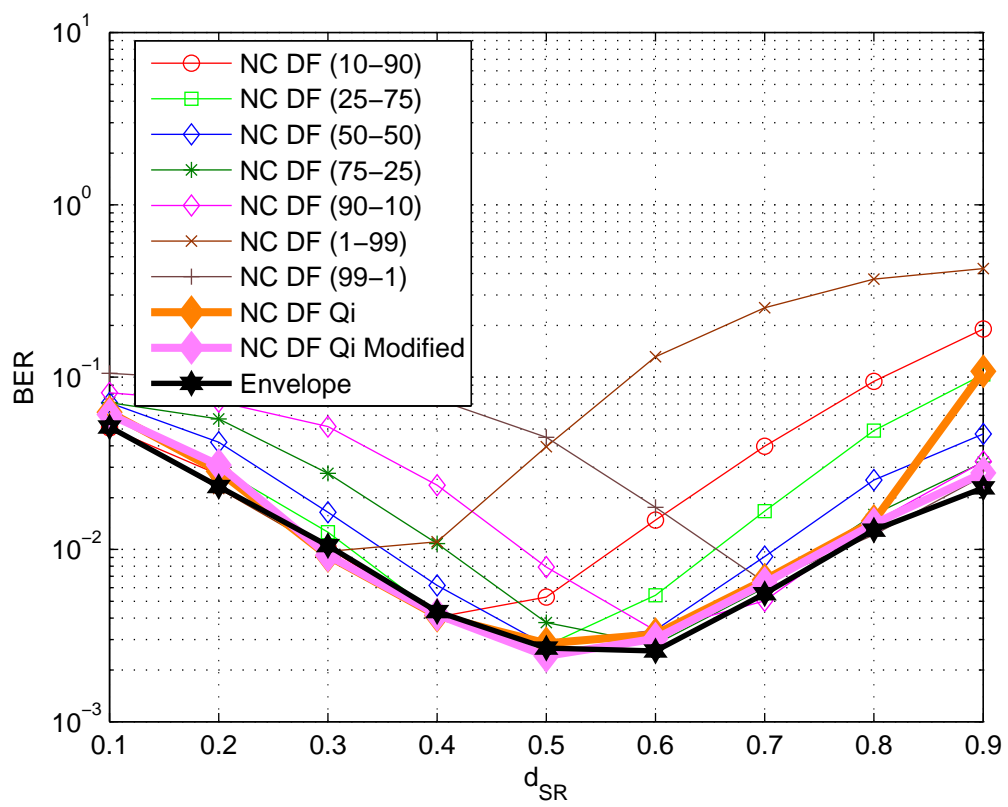

Figure 5.7: Comparison of simulated BER performance of DF with various different power allocations in Phase 1 and Phase 2 and Qi's original and modified power allocation scheme. The envelope represents the best $\mathrm{BER}$ performance given by $\min _{p_{1}, p_{2}} \operatorname{BER}\left(p_{1}, p_{2}, d\right)$.

than direct communication as the relay moves farther away from the source or destination and it is in those region relaying would be of no consequence. To verify this idea we move on from the collinear case and simulate the system performance over a grid around the source and destination.

But before that we will see how much of a difference does increasing the received SNR makes. To this end, we repeat the the collinear scenarios for noncoherent AF and DF for a received SNR of $20 \mathrm{~dB}$ and present the results in Fig. 5.8. Since AF and DF have same performance they are represented by the same curve. There is definitely a considerable improvement in performance with increase of SNR but we also notice that there is no major change in the shape of the BER curves for different SNRs. Using optimal power allocation, the performance of direct communication is almost as good or better than the relay communication near the source. But near the destination our relay communication performs better than the direct communication even for different received SNRs. The throughput for higher SNR, as seen in Fig. 5.9, is also generally higher but there is a some change in the shape and it does not deteriorate as much near the source or relay. 


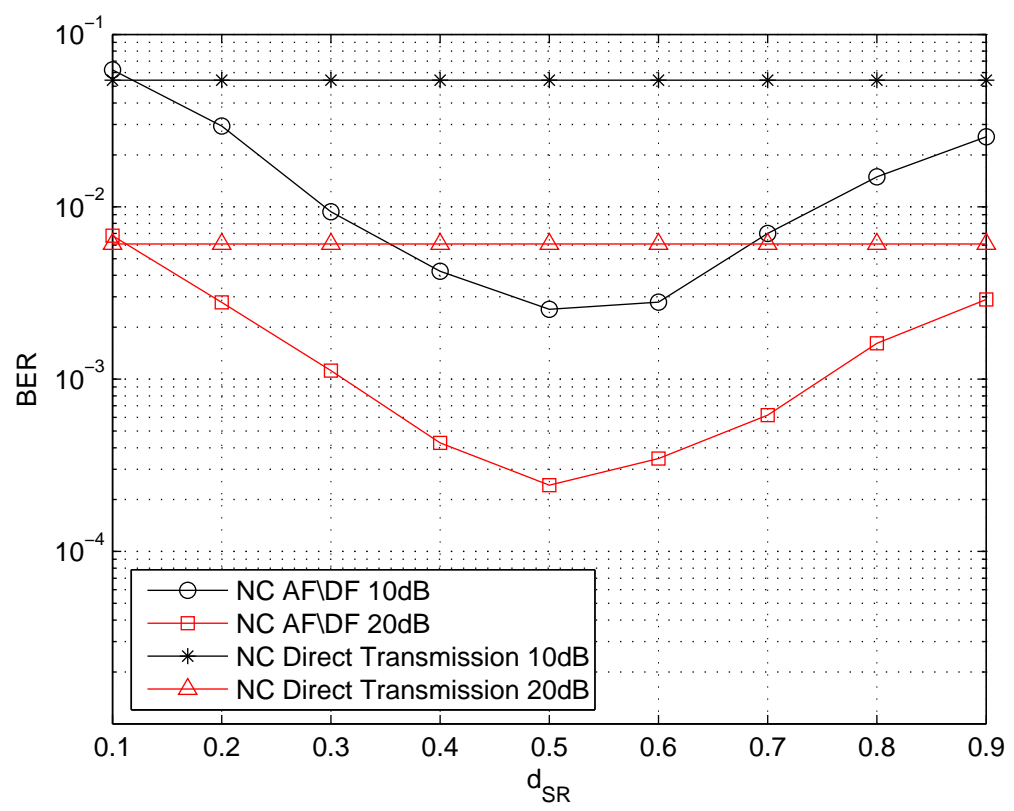

Figure 5.8: Comparison of BER performances of AF and DF with power allocation modified from Qi's, and direct transmission for received SNRs of $10 \mathrm{~dB}$ and $20 \mathrm{~dB}$.

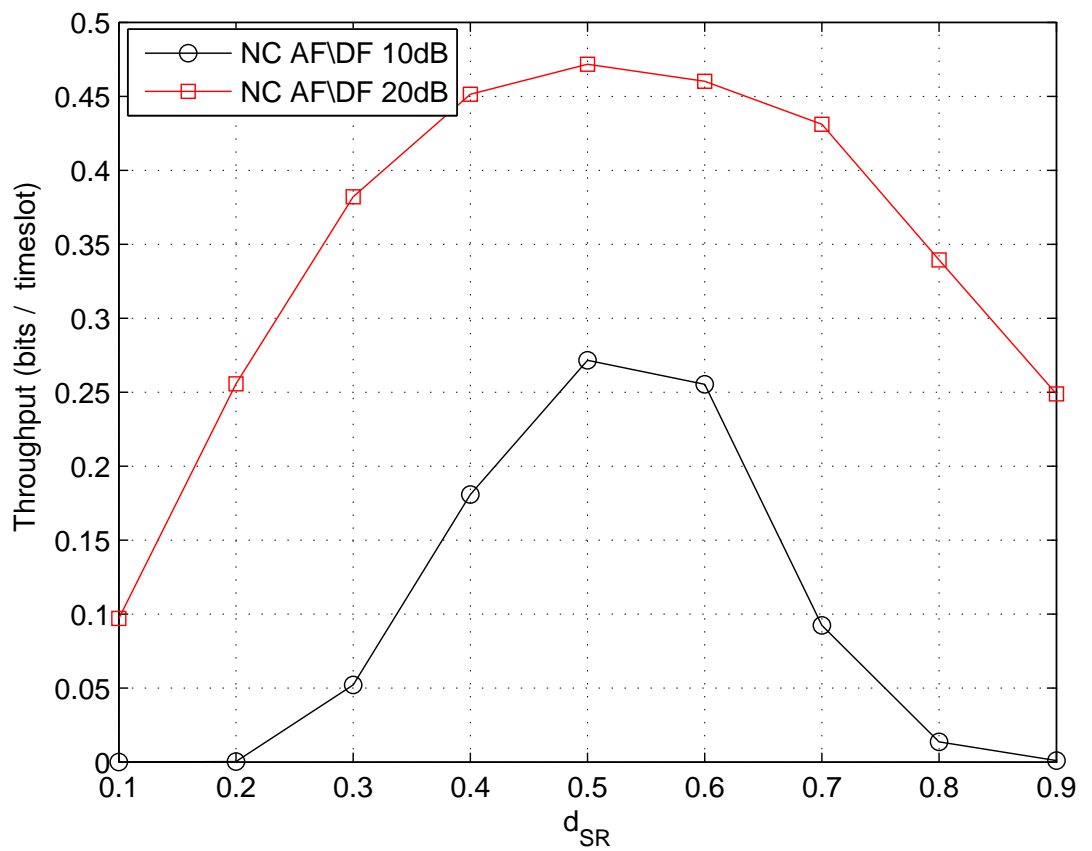

Figure 5.9: Comparison of throughput performances of AF and DF with power allocation modified from Qi's, and direct transmission for received SNRs of 10dB and 20dB. 


\subsection{Power Optimization Over a Grid and Area of In- terest}

As discussed in the last section we will simulate the performance of both the forwarding protocols, $\mathrm{AF}$ and $\mathrm{DF}$, over a $3 \times 3$ plane containing the three nodes viz. the source, the destination and the relay with the relay free to move over the plane. The received SNR is considered to be $10 \mathrm{~dB}$. The Fig. 5.10 and Fig. 5.11 shows the performance of AF and DF respectively over the grid and that of the direct communication. Again we notice that both the protocols perform almost equally in the noncoherent modulation scenario.

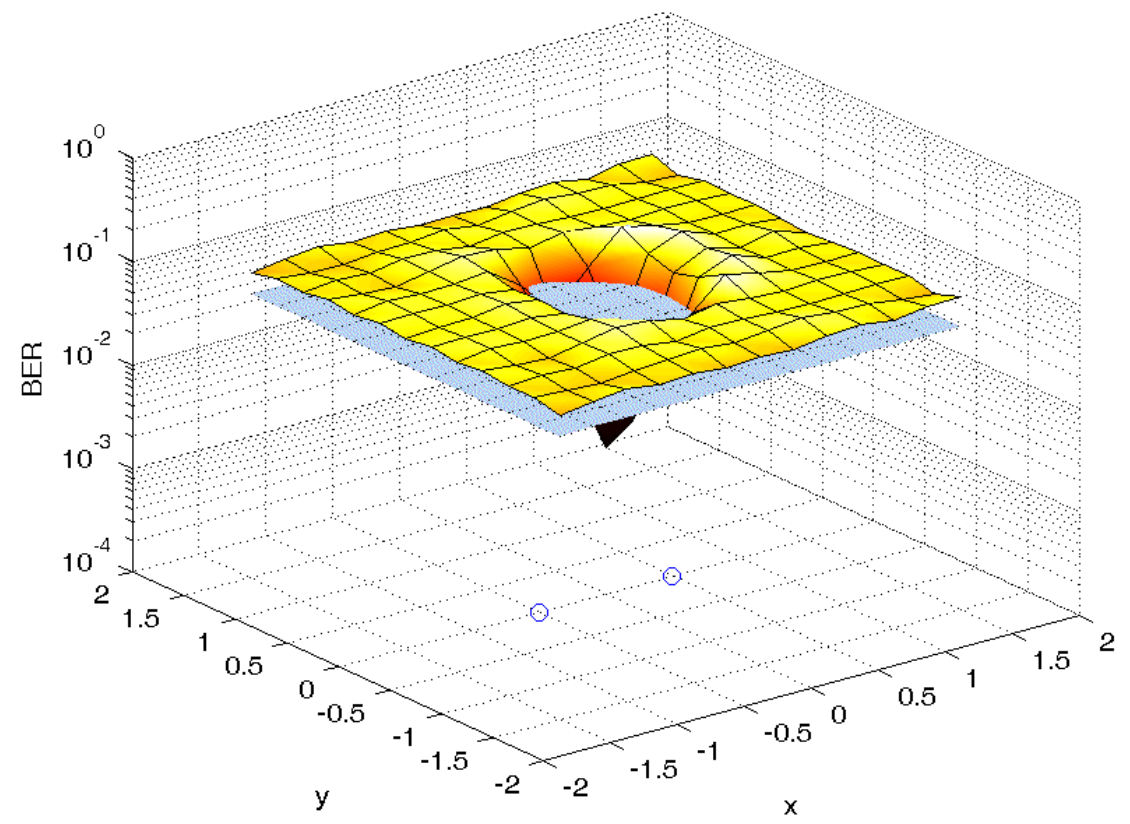

Figure 5.10: BER performance of AF, with power allocation modified from Qi's, and direct transmission. The received $\mathrm{SNR}$ is $10 \mathrm{~dB}$. Source at $\mathrm{x}, \mathrm{y}=(-0.5,0)$ and destination at $\mathrm{x}, \mathrm{y}=$ $(0.5,0)$.

As in the collinear case, we will see how the BER performance behaves over the grid when the system has different received SNRs in Fig. 5.12. Both the AF and DF, have essentially similar performances so we present the performances of DF as the representative one. From the Fig. 5.12 it can be clearly seen that the BER performance achieved at the higher SNRs is predictably better than that for the lower one. We also plot the throughput per timeslot, for both the SNRs in Fig. 5.12 and notice that, generally the throughput is lower than the 


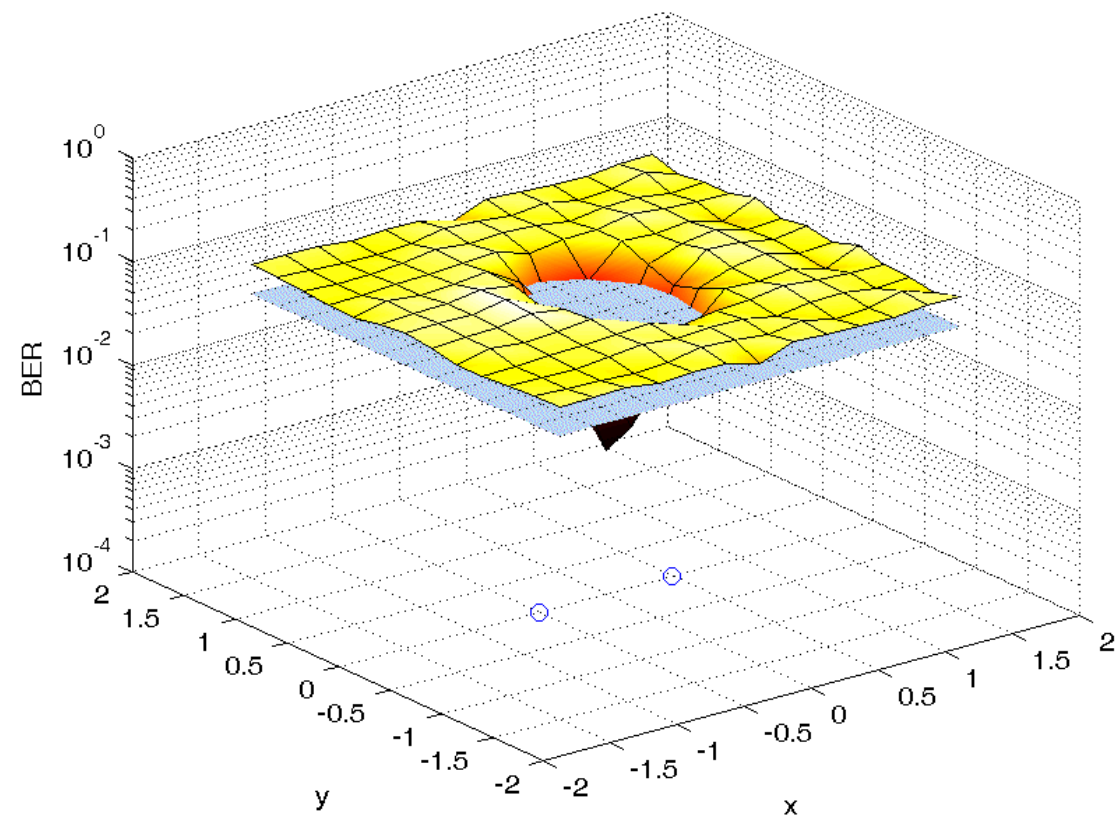

Figure 5.11: BER performance of DF, with power allocation modified from Qi's, and direct transmission. The received SNR is $10 \mathrm{~dB}$. Source at $\mathrm{x}, \mathrm{y}=(-0.5,0)$ and destination at $\mathrm{x}, \mathrm{y}=$ $(0.5,0)$.

coherent case, specially so for areas relatively farther away from the source and destination. Also, for a higher SNR the throughput is markedly better, that too over a wider region than the lower SNR.

To identify the regions where relaying actually helps in AF or DF, we look at Fig. 5.10 and Fig. 5.11, respectively, from the top. Analyzing Fig. 5.14 and Fig. 5.15 we see that there is a certain oval shaped area around the region between the source and destination, almost identical for both AF and DF, where a performance gain over direct transmission is achieved by using a relay.

Using our system model we see that the performance of AF and DF are almost identical in noncoherent scenario. As in previous chapters, we proceed to check if there is a change in the area, where relaying is relevant, with changes in received SNR and represent it in Fig. 5.16. The left hand plot of the figure represents the scenario when received SNR is $10 \mathrm{~dB}$ and the right hand one that for $20 \mathrm{~dB}$. It is evident from this figure that there is almost no change in area where relaying is helpful in the noncoherent scenario. This indicates that the BER performance of the relay system and that of direct communication change in such 


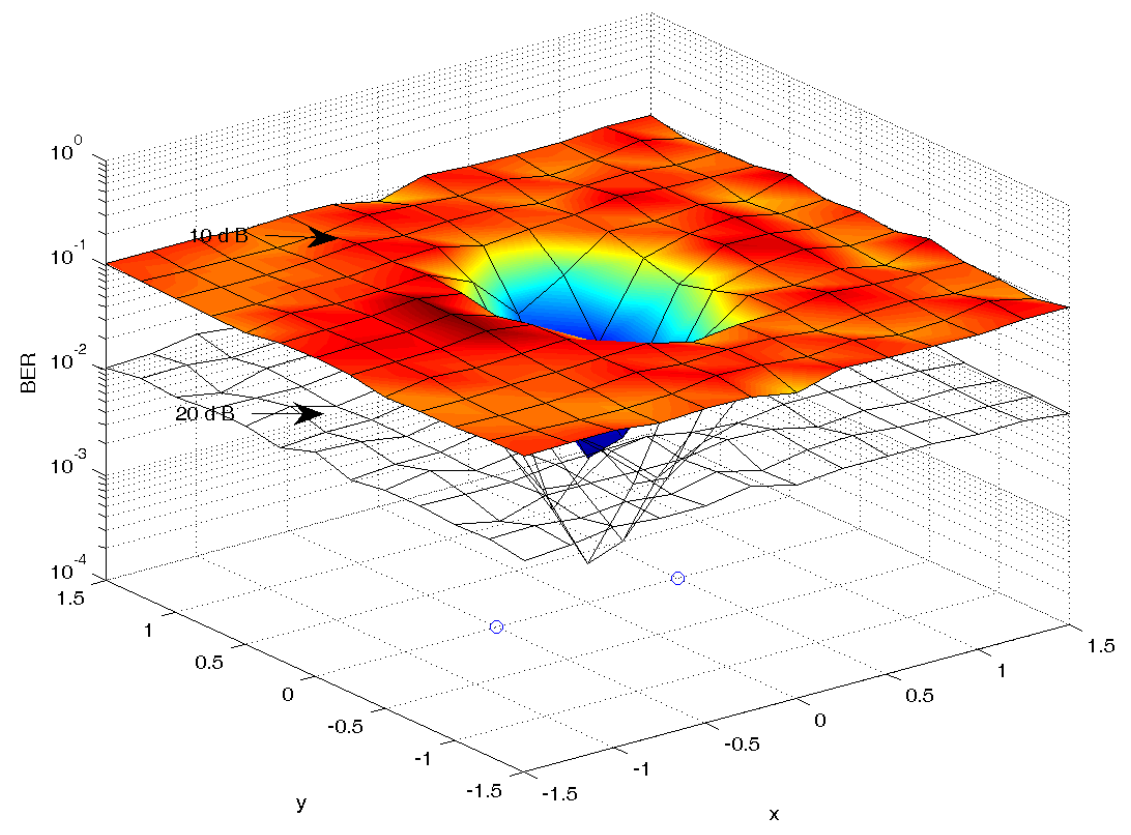

Figure 5.12: BER performances of noncoherent DF, with power allocation modified from Qi's with received SNRs of $10 \mathrm{~dB}$ and $20 \mathrm{~dB}$. Source at $\mathrm{x}, \mathrm{y}=(-0.5,0)$ and destination at $\mathrm{x}, \mathrm{y}$ $=(0.5,0)$.

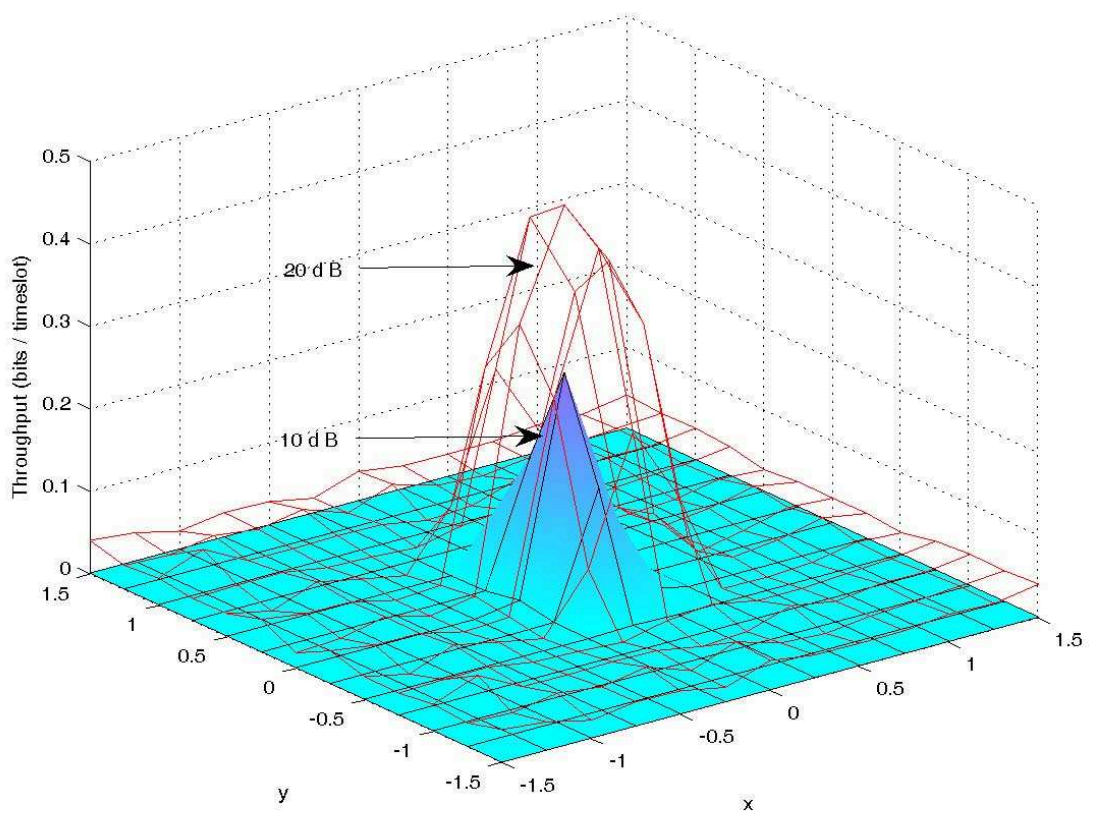

Figure 5.13: Throughput performance of noncoherent DF, with power allocation modified from Qi's with received SNRs of $10 \mathrm{~dB}$ and $20 \mathrm{~dB}$. Source at $\mathrm{x}, \mathrm{y}=(-0.5,0)$ and destination at $\mathrm{x}, \mathrm{y}=(0.5,0)$. 


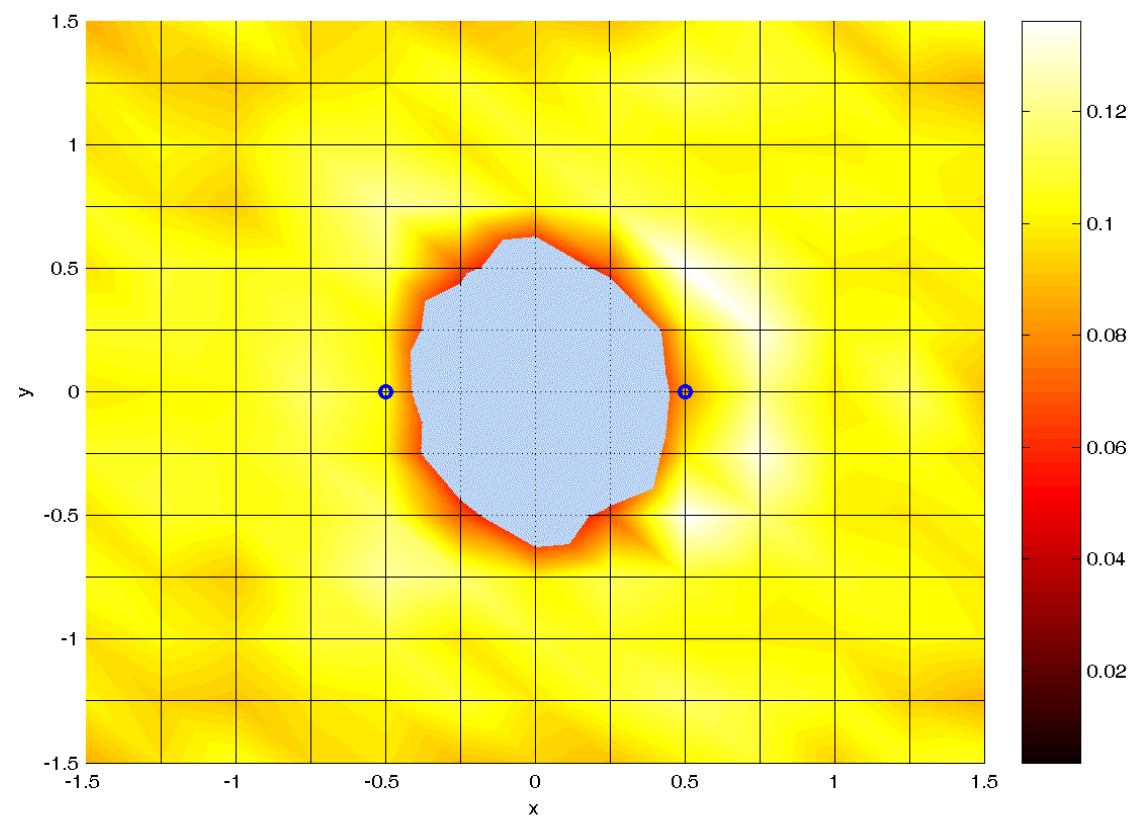

Figure 5.14: The inner lighter area represents the region for relaying in AF with noncoherent modulation. The received SNR is $10 \mathrm{~dB}$. Source at $\mathrm{x}, \mathrm{y}=(-0.5,0)$ and destination at $\mathrm{x}, \mathrm{y}=$ $(0.5,0)$.

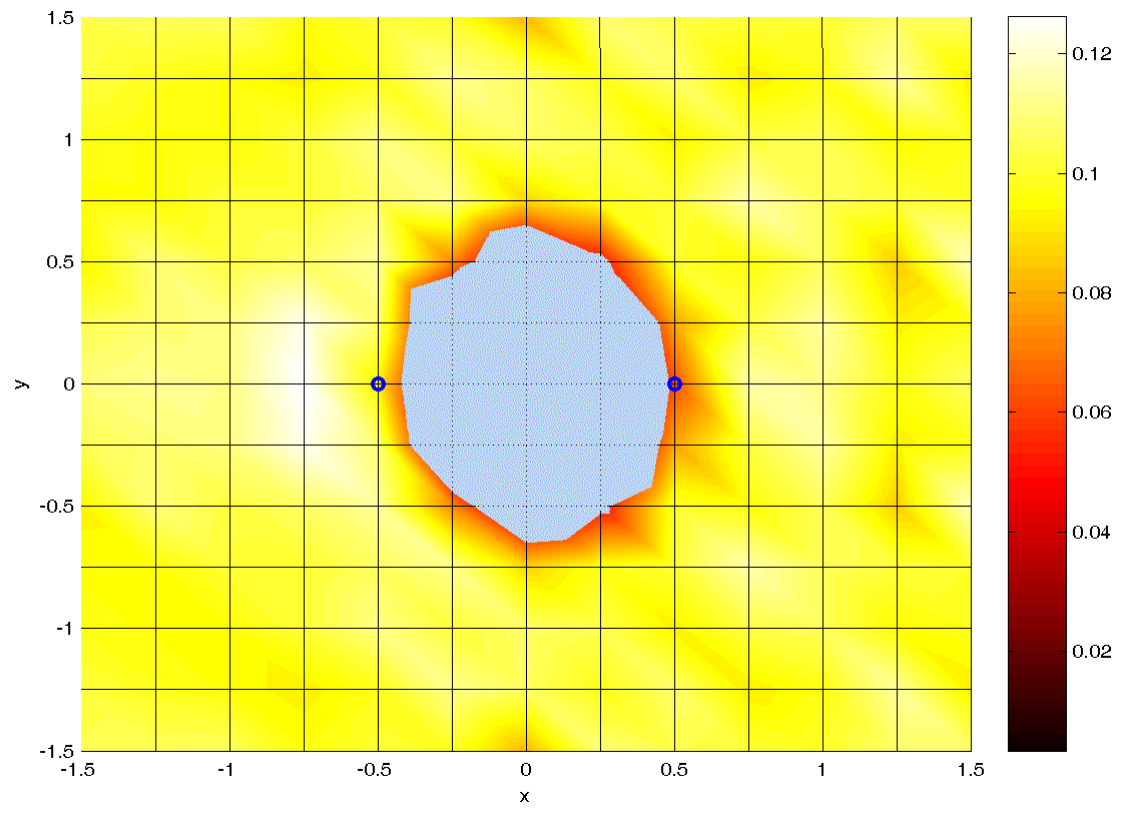

Figure 5.15: The inner lighter area represents the region for relaying in DF with noncoherent modulation. The received SNR is $10 \mathrm{~dB}$. Source at $\mathrm{x}, \mathrm{y}=(-0.5,0)$ and destination at $\mathrm{x}, \mathrm{y}=$ $(0.5,0)$. 
a way with changing SNR that it has no effect on the area of interest, where relaying is helpful. Fig. 5.17 shows the same thing for DF. As before the left hand plot shows the area of interest for noncoherent DF with 10dB received SNR and the right hand one shows that for $20 \mathrm{~dB}$ received SNR. Comparing Fig. 5.16 and Fig. 5.17 we can see that AF and DF behaves almost identically with change in received SNR and has almost similar areas where relaying is useful.
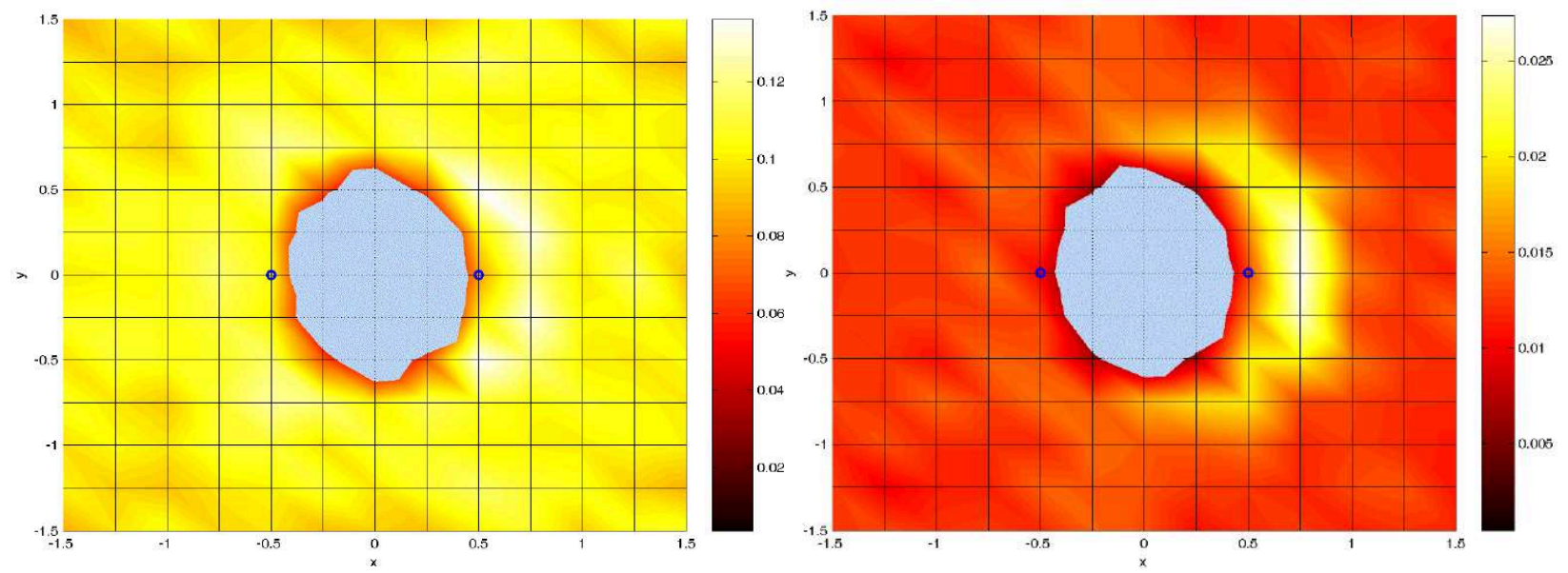

Figure 5.16: The inner lighter areas represent the region for relaying in noncoherent environment AF. The received SNR for the left hand plot is $10 \mathrm{~dB}$ and that for the right hand one is $20 \mathrm{~dB}$. Source at $\mathrm{x}, \mathrm{y}=(-0.5,0)$ and destination at $\mathrm{x}, \mathrm{y}=(0.5,0)$.
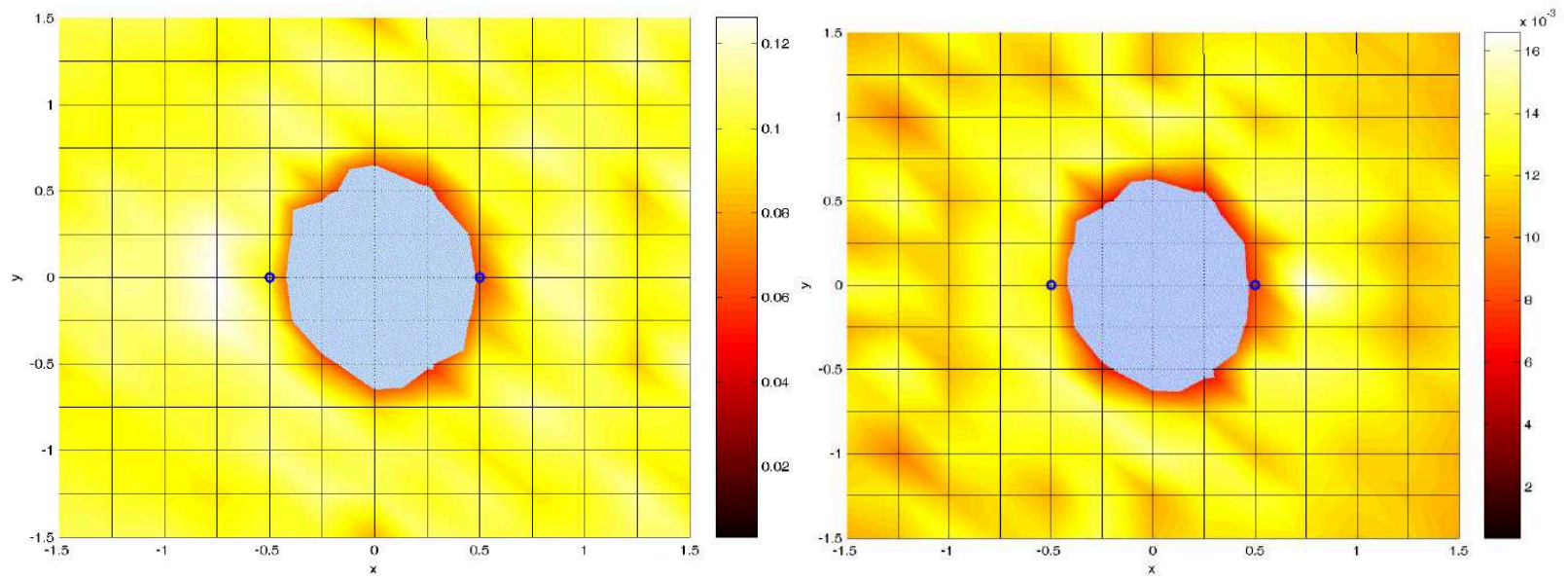

Figure 5.17: The inner lighter areas represent the region for relaying in noncoherent environment using DF. The received SNR for the left hand plot is $10 \mathrm{~dB}$ and that for the right hand one is $20 \mathrm{~dB}$. Source at $\mathrm{x}, \mathrm{y}=(-0.5,0)$ and destination at $\mathrm{x}, \mathrm{y}=(0.5,0)$. 


\subsection{Conclusion}

In a scheme with noncoherent demodulation one would normally take into account some inherent performance penalties. But using power optimization, in a relay environment with noncoherent demodulation, we are able to show that there is performance benefits at certain areas, specially in the general area between the source and destination. Here too, as in the coherent case, power optimized schemes perform better than arbitrary power allocations. It was also noticed that the performance of $\mathrm{AF}$ and $\mathrm{DF}$, with power optimization, are very close and hence probably it is a good idea to use AF, the less complex of the two, at the relay with almost none or minimal performance penalty. It was also noticed that with different received SNRs, the area where relaying improves performance, stays almost the same. So there is a particular region, valid for a range of moderate SNRs, where the relay should be positioned to achieve performance gains. Also as seen in the coherent case, we observe that the best performance is extracted from the system when the relay is in the midway region between the source and the destination, making that a target area to place the relay in practical scenarios. 


\section{Chapter 6}

\section{Conclusions}

\subsection{Summary and Conclusions}

We started this work with the preliminary goal to investigate the behavior of a relay network, with a mobile relay and concluded by proposing a position dependant optimal power allocation scheme. Both coherent and noncoherent demodulation were considered along with practical modulation schemes. In both the scenarios we evaluated the performance of AF and DF techniques. It was seen that in the coherent environment DF performed better than AF generally but in the noncoherent case the performance of the two forwarding protocols were almost the same. Keeping in mind these results, we went on optimizing the power allocation in the different transmit phases, between the transmitter and the relay. For the coherent case we optimized the power allocation, based on the combined instantaneous SNR of the system and saw that this scheme outperforms other arbitrary power allocations and also some existing schemes using information theoretic approaches. For the noncoherent case we used a slightly modified version of Qi et. al.'s power allocation scheme, adapted to our system and observed that here too the position based power optimization outperforms other arbitrary power allocations. We also identified the areas of interest where relaying is relevant for all these scenarios. It was noticed that in coherent environment, AF has a cardioid shaped area where relaying is useful, whereas DF has a elliptical area. In the coherent case, for the same received SNR, the size of this area of interest is bigger for DF than AF. With increasing received SNR in the coherent case, the size of these areas for both 
AF and DF increased. Their shape remained the same and the DF still had a bigger area where relaying would be useful. While trying to see the same effects in the noncoherent case, we found that for both AF and DF, the area where relaying is beneficial, is almost of the same size and shape. Another interesting result was that for higher SNRs the performance of power optimized AF and DF improves but the shape and size of the area of interest does remains almost the same. It is a known fact that the when the relay is placed around the midway region between the source and the destination, the best overall performance is achieved. We observed that this fact is true for our position dependant power optimized scheme too.

To sum it up we propose that DF with our power optimized scheme should be used in coherent cases, as it offers overall BER and throughput improvements. While using our model in the noncoherent case however, using the simpler and cheaper to implement AF would suffice, since DF does not offer any practical improvements here.

\subsection{Future Work}

While working on the thesis we observed that some of the nonoptimal power allocation schemes work differently in $\mathrm{AF}$ and $\mathrm{DF}$ at certain areas, but the optimal power allocation is very similar for both the protocols, pointing to the fact that there are certain powers which perform best in both AF and DF for a particular area. This, with further studies, might lead into a better understanding about the effect and importance of power allocation in cooperative relay networks. More research can be done to come up with analytical results in the noncoherent case. The effect of multiple relays could also be evaluated. We did some work on orthogonal multiple relays and initial results showed promise with improved BER but the orthogonality introduces system latency and reduces throughput. Further work could be done to mitigate this problem. It would also be interesting to know if there is a particular and practical SNR regime in which relaying is important. We have done some preliminary work with the incorporation of convolutional codes in the system along with Viterbi decoding. The behavior of the system with coding, under the various conditions considered would be an interesting insight. 


\section{References}

[1] R. Ahlswede, "Multi-way communication channels," in Proc. IEEE Int. Symp. on Inform. Theory (ISIT), Thakadsor, USSR, Sept. 1971, pp. 105-135. 1

[2] J. Winters, "On the capacity of radio communication systems with diversity in a Rayleigh fading environment," IEEE J. Select. Areas Commun., vol. 5, pp. 871-878, June 1987. 1

[3] G. J. Foschini, "Layered space-time architecture for wireless communication in fading environments when using multi-element antennas," Bell Labs Technical Journal, Autumn 1996. 1

[4] G. J. Foschini and M. Gans, "On the limits of wireless communications in a fading environment when using multiple antennas," Wireless Personal Commun., vol. 6, no. 3, pp. 311-335, Mar. 1998. 1

[5] P. F. Driessen and G. J. Foschini, "On the capacity formula for multiple input-multiple output wireless channels: A geometric interpretation," IEEE Trans. Commun., vol. 47, no. 2, pp. 173 - 176, Feb. 1999. 1

[6] E. Telatar, "Capacity of multi-antenna Gaussian channels," ATEST-Bell Labs Internal Memo, June 1995. 1

[7] E. Telatar, "Capacity of multi-antenna Gaussian channels," European Trans. on Telecommun. (ETT), vol. 10, pp. 585-596, Nov. 1999. 1

[8] S. Alamouti, "A simple transmit diversity technique for wireless communications," IEEE J. Select. Areas Commun., vol. 16, no. 8, pp. 1451-1458, Oct. 1998. 1

[9] V. Tarokh, H. Jafarkhani, and A. R. Calderbank, "Space-time block codes from orthogonal designs," IEEE Trans. Inform. Theory, vol. 45, no. 5, pp. 1456-1467, July 1999. 1

[10] V. Tarokh, H. Jafarkhani, and A. R. Calderbank, "Space-time block coding for wireless communications: Performance results," IEEE Trans. Inform. Theory, vol. 17, no. 3, pp. 451-460, Mar. 1999. 1

[11] V. Tarokh, N. Seshadri, and A. R. Calderbank, "Space-time codes for high data rate wireless communications: Performance criterion and code construction," IEEE Trans. Inform. Theory, vol. 44, no. 2, pp. 744-765, Mar. 1998. 1 
[12] F. A. Tobagi, "Modeling and performance analysis of multihop packet radio networks," in Proc. IEEE, pp. 135-155, Jan. 1987. 1

[13] M.B. Pursley, "The role of spread spectrum in packet radio networks," in Proc. IEEE , pp. 116-134, Jan. 1987. 1

[14] L. Kleinrock and J. Silvester, "Optimum transmission radii for packet radio networks or why six is a magic number," in Proc. IEEE Natl. Telecomm. Conf., pp. 4.3.1- 4.3.5, Dec. 1978. 1

[15] A. Ephremides, J. E. Wieselthier, and D. J. Baker, "A design concept for reliable mobile radio networks with frequency hopping signaling.," in Proc. IEEE , pp. 56-73, Jan. 1987. 1

[16] R. E. Kahn, S. A. Gronemeyer, J. Burchfiel, and R. C. Kunzelman, "Advances in packet radio technology," in Proc. IEEE, vol. 66, pp. 1468-1496, Nov. 1978. 1, 17

[17] A. Goldsmith and S. Wicker, "Design challenges for energy-constrained ad hoc wireless networks," IEEE Wireless Communications, vol. 9, no. 4, pp. 8-27, Aug. 2002. 1

[18] A. Agarwal and P. R. Kumar, "Capacity bounds for ad hoc and hybrid wireless networks," ACM SIGCOMM Computer Communication Review, vol. 34, July 2004. 1

[19] S. Cui, A. J. Goldsmith and A. Bahai, "Energy-efficiency of MIMO and cooperative MIMO in sensor networks," IEEE J. Select. Areas Commun., vol. 22, no. 6, Aug. 2004. 2

[20] I. Maric and R. D. Yates, "Cooperative multihop broadcast for wireless networks," IEEE J. Select. Areas Commun., vol. 22, no. 6, pp. 1080-1088, Aug. 2004. 2

[21] A. Sendonaris, E. Erkip, B. Aazang, "User cooperative diversity - Part I: System description," IEEE Trans. Commun., vol. 51, no. 1, pp. 1927-1938, Nov. 2003. 2

[22] A. Sendonaris, E. Erkip, B. Aazang, "User cooperative diversity - Part II: Implementation aspects and performance analysis," IEEE Trans. Commun., vol. 51, no. 1, pp. 1939-1948, Nov. 2003. 2

[23] G. Kramer, M. Gastpar, and P. Gupta, "Cooperative strategies and capacity theorems for relay networks," IEEE Trans. Inform. Theory, vol. 51, no. 9, pp. 3037-3063, Sept. 2005. 3

[24] M. Gastpar and M. Vetterli, "On the capacity of large Gaussian relay networks," IEEE Trans. Inform. Theory, vol. 51, no. 3, pp. 765-779, Mar. 2005. 3

[25] J. N. Laneman, D. N. C. Tse, and G. W. Wornell, "Cooperative diversity in wireless networks: Efficient protocols and outage behavior," IEEE Trans. Inform. Theory, vol. 50, no. 12 , pp. 3062-3080, Dec. 2004. 3 
[26] Y. W. Ding, J. K. Zhang and K. M. Wong, "The amplify and forward half duplex cooperative system: Pairwise error probability and precoder design," IEEE Trans. Inform. Theory, vol. , to appear. 3

[27] R. U. Nabar, F. W. Kneubühler and H. Bölcskei , "Performance limits of Amplify and Forward based fading relay channels," in Proc. Int. Conf. on Acoustics, Speech and Signal Processing (ICASSP), May 2004. 3

[28] S. Yang and J.C. Belfiore, "Towards the optimal Amplify and Forward cooperative diversity scheme," IEEE Trans. Inform. Theory, Mar. 2006, submitted for publication. 3

[29] X. Bao and J. Li, "Decode-Amplify-Forward (DAF): A new class of forwarding strategy for wireless relay channels," in Proc. of IEEE Workshop on Signal Processing Advances in Wireless Communications (SPAWC), New York, NY, June 2005. 3, 22

[30] M. Yu, T. Li and H.R. Sadjadpour, "Amplify-Forward and Decode-Forward: The impact of location and capacity contour," in Proc. IEEE Military Commun. Conf. (MILCOM ), 2005. 3

[31] A. Goldsmith, Wireless Communications, Publisher: Cambridge University Press, ISBN: 0521837162, 08 August 2005. 4, 21, 58

[32] W. Zhang and J. N. Laneman, "Benefits of spatial correlation for multi-antenna noncoherent communication over fading channels at low SNR," IEEE Trans. Wireless Comm., vol. , Jan. 2006, revised and submitted for publication. 5]

[33] Y. Liang and V. V. Veeravalli, "Gaussian orthogonal relay channel: Optimal resource allocation and capacity," IEEE Trans. Inform. Theory, Sept. 2005, to appear. 5, 23

[34] M. O. Hasna and M. S. Alouini, "Optimum power allocation for selective transmitdiversity systems over Nakagami fading channels," in Proc. Int. Conf. on Acoustics, Speech and Signal Processing (ICASSP), Orlando, Florida, USA, May 2002. 5, 23

[35] A. Reznik, S. Kulkarni and S. Verdú, "Degraded Gaussian multi-relay channel: Capacity and optimal power allocation," IEEE Trans. Inform. Theory, vol. 50, no. 10, pp. 30373046, Dec. 2004, to appear. 5, 22

[36] P. Larsson, "Large-scale cooperative relaying network with optimal coherent combining under aggregate relay power constraint," in Proc. Future Telecommunications Conference, Beijing, 2003. 5, 23

[37] Z. Qi, Z. Jingmei, S. Chunju, W. Ying, Z. Ping and H. Rong, "Power allocation for regenerative relay channel with Rayleigh fading," in Proc. IEEE Veh. Tech. Conf. (VTC), vol. 2, Spring 2004. 5, 23, 53, 54, 62, 63

[38] J.G. Proakis, Digital Communications: Third Edition, New York, NY: McGraw-Hill, ISBN:0072321113, 1995. 10, 16, 17, 27 
[39] F. Gray, "Pulse code communication," U.S. patent no. 2,632,058, Mar.17 1953. 12

[40] T. Rappaport, Wireless Communication Systems: Third Edition, New York, NY:McGraw-Hill, ISBN:0130422320, 1995. 13, 17, 19, 20, 21, 27, 80

[41] R. E. Ziemer and R. L. Peterson, Introduction to Digital Communications, Macmillan Publishing Company, 1992. 15

[42] D. Reynolds, "Wireless communication systems," Course Notes, WVU, Fall 2003. 17, 19

[43] D.C. Cox, R.Murray and A. Norris, " $800 \mathrm{MHz}$ attenuation measured in and around suburban houses," AT and T Bell Laboratory Technical Journal, vol. 673, no. 6, JulyAug. 1984. 21

[44] R.C. Bernhardt, "Macroscopic diversity in frequency reuse systems," IEEE J. Select. Areas Commun., vol. SAC-5, no. 6, pp. 862-878, June 1987. 21

[45] C. E. Shannon, "A mathematical theory of communication," Bell System Technical Journal, vol. 27, no. 6, pp. 379 to 423 and 623 to 656, July and Oct. 1948. 22

[46] R.M. Fano, "A heuristic discussion of probabilistic decoding," IEEE Trans. Inform. Theory, vol. IT-9, no. 2, pp. 64-74, Apr. 1963. 22

[47] E. C. van der Meulen, "Three-Terminal communication channels," Adv. Appl. Probab., vol. 3, pp. 120-154, 1971. 22

[48] T. M. Cover and A. A. El Gamal, "Capacity theorems for the relay channel," IEEE Trans. Inform. Theory, vol. IT-25, no. 5, pp. 572-584, Sept. 1979. 22

[49] T. M. Cover, "Broadcast Channels," IEEE Trans. Inform. Theory, vol. IT-18, no. 1, pp. 2-4, Jan. 1972. 22

[50] T. M. Cover, A. El Gamal, and M. Salehi, "Multiple access channels with arbitrarily correlated sources," IEEE Trans. Inform. Theory, vol. IT-26, pp. 648-657, Nov. 1980. 22

[51] T. M. Cover, R. J. McEliece, and E. C. Posner, "Asynchronous Multiple Access Channel Capacity," IEEE Trans. Inform. Theory, vol. IT-27, pp. 409-413, July 1981. 22

[52] A. A. El Gamal, M. R. Aref, "The capacity of the semideterministic relay channel," IEEE Trans. Inform. Theory, vol. 28, no. 3, pp. 536, July 1982. 22

[53] E. van der Meulen, "Introduction of the relay channel," MSRI Workshop: Mathematics of Relaying and Cooperation in Communication Networks, Apr. 10-12 2006. 22

[54] I. Maric and R. Yates, "Forwarding strategies for Gaussian parallel-relay networks," in Conf. on Inform. Sciences and Sys. (CISS), Mar. 2004. 23 
[55] P. Herhold, E. Zimmermann and G. Fettweis, "A simple cooperative extension to wireless relaying," 2004 Int. Zurich Seminar on Communications, Zurich, Switzerland, Feb. 2004, accepted. 23

[56] S. Datta Gupta and D. Reynolds, "Position dependant power allocation strategies in cooperative relay networks," in IEEE Military Commun. Conf. (MILCOM), Washington, DC, USA, Oct. 2006. 24, 40

[57] D. J. Goodman and N. B. Mandayam, "Power control for wireless data," IEEE Personal Commun., vol. 7, no. 2, pp. 48-54, Apr. 2000. 26

[58] D. P. Bertsekas, Nonlinear Programming: Second Edition, Belmont, MA: Athena Scientific, ISBN:1886529000 , 1999. 32, 45, 83

[59] D. F. Shanno, "Conditioning of Quasi-Newton methods for function minimization," Mathematics of Computation, vol. 24, no. 111, pp. 647-656, July 1970, Mathematics of Computation is currently published by American Mathematical Society. 32, 45, 83

[60] S. Verdú, Multiuser Detection, Cambridge, UK: Cambridge University Press, ISBN:0521593735, 1998. 80

[61] A. Leon-Garcia, Probability and Random Processes for Electrical Engineering: Second Edition, Addison-Wesley Publishing Company,Inc., ISBN:020150037X, 1994. 81

[62] A. S. Tanenbaum, Computer Networks: Fourth Edition, Prentice Hall India, New Delhi-110001,India, ISBN:8120321758, 2002. 82

[63] T. Moon and W. Stirling, Mathematical Methods and Algorithms for Signal Processing, Prentice-Hall, Upper Saddle River, NJ, USA, ISBN:0201361868, 2000. 83 


\section{Appendix}

\section{A Q-Function}

Q-function act as a very handy tool for determining the theoretical probability of error in a host of communication processes $[40,60]$. Finding the area under the tail of the Gaussian or Normal probability density function is required when dealing with probabilities of Gaussian processes. This is shown by the left hand side plot in Fig. .1. It shows that the probability of a Gaussian random variable $x$, with mean $m$ and variance $\sigma^{2}$, exceeding $x_{0}$ is given by

$$
\operatorname{Pr}\left(x \geq x_{0}\right)=\int_{x_{0}}^{\infty} \frac{1}{\sigma \sqrt{2 \pi}} \mathrm{e}^{\frac{-(x-m)^{2}}{2 \sigma^{2}}} d x
$$

It is seen that $(\mathrm{A}-1)$ is not integrable in closed form. Now using the substitution, $y=\frac{(x-m)}{\sigma}$
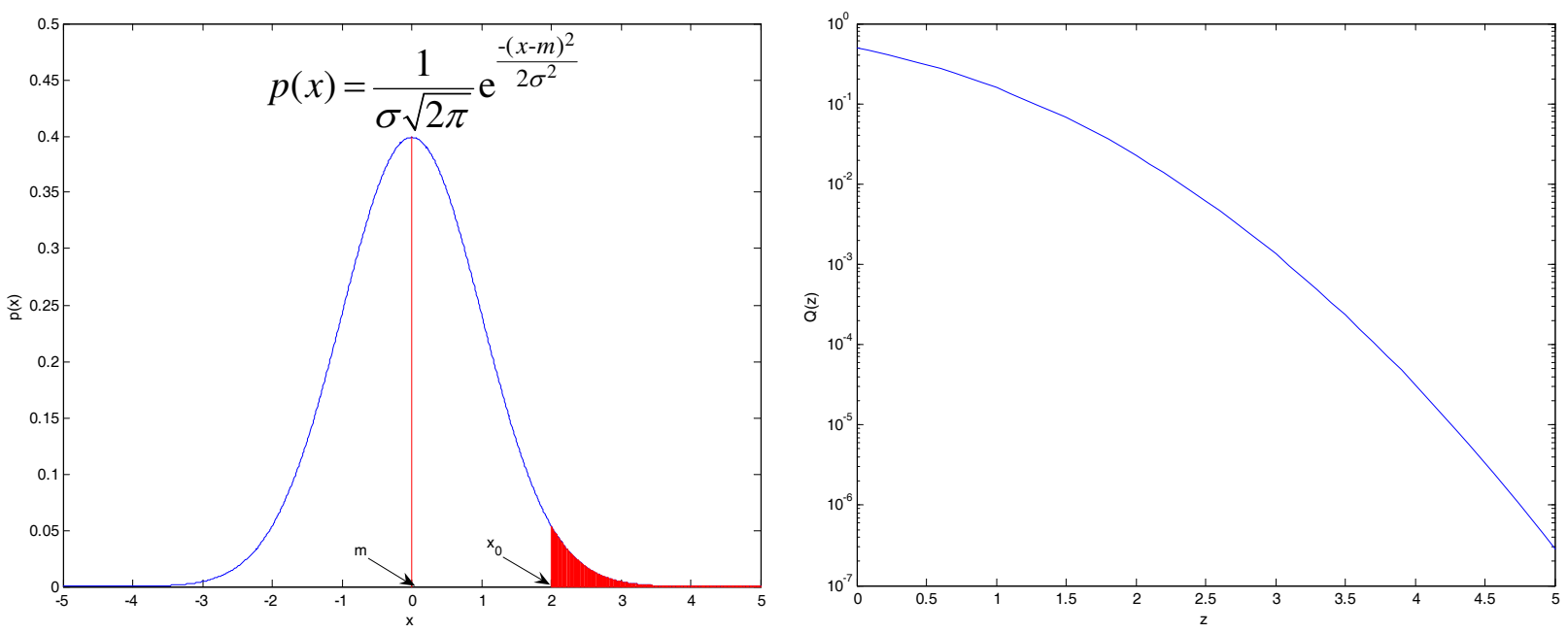

Figure .1: The left hand plot shows the Gaussian probability density function with 0 mean and standard deviation 1 . The shaded area represents the area under the tail of a Gaussian random variable i.e. $\operatorname{Pr}\left(x \geq x_{0}\right)$. The right hand plot represents the plot of the Q-function. 
and $z=\frac{(x-m)}{\sigma}$, the integral in A-1 can be evaluated as,

$$
Q(z)=\int_{z}^{\infty} \frac{1}{\sqrt{2 \pi}} \mathrm{e}^{\frac{-y^{2}}{2}} d y .
$$

It is this equation, (A-2), which is known as Q-function. The right hand plot shows the plot of the Q-function. A couple of important properties of it are,

$$
\begin{array}{r}
Q(-z)=1-Q(z) \\
Q(0)=\frac{1}{2} .
\end{array}
$$

There is a relation between Q-function and error function and complimentary error function which sometimes come in handy in numerical evaluation of Q-function. The error function can be defined as,

$$
\operatorname{erf}(z)=\frac{2}{\sqrt{\pi}} \int_{0}^{z} \mathrm{e}^{-x^{2}} d x
$$

and the complimentary error function can be defined as,

$$
\operatorname{erfc}(z)=\frac{2}{\sqrt{\pi}} \int_{z}^{\infty} \mathrm{e}^{-x^{2}} d x
$$

The relation between erf and $\operatorname{erfc}$ and Q-function is given by

$$
\begin{gathered}
Q(z)=\frac{1}{2}\left[1-\operatorname{erf}\left(\frac{z}{\sqrt{2}}\right)\right] \\
=\frac{1}{2} \operatorname{erf}\left(\frac{z}{\sqrt{2}}\right) .
\end{gathered}
$$

\section{B Bernoulli Random Variable}

Bernoulli random variable is a discrete random variable, which takes value 1 with probability $p$ and value 0 with probability $q=1-p[61]$. If we define $A$ as the event depending on the results of some random experiment then the indicator function for $A$ can be defined as

$$
I_{A}(\eta)= \begin{cases}0 & \text { if } \eta \text { not in } A \\ 1 & \text { if } \eta \text { in } A\end{cases}
$$

So $I_{A}$ is a discrete random variable since it assigns discrete numbers to each outcome of the experiment and has a range $S_{X}=\{0,1\}$. Its probability mass function is $p_{I}(0)=1-p$ 
and $p_{I}(1)=p$. Now if we associate $I_{A}=1$ with a success, we can define $P(A)=p I_{A}$ as a Bernoulli random variable. We have modeled the event of the relay decoding a signal correctly, as a Bernoulli random variable, in our thesis.

\section{Cyclic Redundancy Check}

Cyclic redundancy check (CRC) is widely used in communication to detect bit errors [62]. Though theoretically it is possible for errors to go undetected even after going through CRC, but CRC using well designed and long enough generator polynomials are robust enough for practical purposes. The generator polynomial $G(x)$ is critical for CRC. The start and end bits of $G(x)$ needs to be 1 and the frame length of the message polynomial, $M(x)$ should be longer than $G(x)$. All the arithmetic is done using modulo 2 for CRC.

To start finding the CRC checksum the same number of zeros as the degree of $G(x)$ is appended to the least significant end of $M(x)$. The resulting polynomial is then divided using modulo 2 by $G(x)$. The remainder obtained is subtracted using modulo 2 from the zero-appended message signal to give the CRC check-summed frame. On the receiver side the check-summed frame is divided again by $G(x)$ using modulo 2. If an error occurs and the error is not divisible by $G(x)$ then that error will be detected. But if the error happens to be divisible by $G(x)$ then it will not be detected.

A CRC code with $r$ check bits will detect all burst errors of length $\leq r$. Also the probability of not detecting an error when the burst length is $(r+1)$ bits is $\frac{1}{2^{r-1}}$ and when the burst is more than $(r+1)$ bit long, the probability of an error going unnoticed is $\frac{1}{2^{r}}$. We have used CRC-5-USB, with the generator polynomial $x^{5}+x^{2}+1$, in our thesis for demonstration purposes. CRC-12, CRC-16-CCITT or CRC-32 used in IEEE 802 protocol are some of the better and commonly used CRC codes. 


\section{Quasi-Newton Method}

Minimization using Quasi-Newton method is a variation of minimization using Newton's method [58, 59, 63]. We assume that a function $f(x)$ is twice differentiable and we define

$$
F(x)=f^{\prime}(x)
$$

So there must be an extremum of $f(x)$ at the point $x^{*}$ where $F\left(x^{*}\right)=0$. Now, if $F^{\prime}\left(x^{*}\right)=$ $f^{\prime \prime}\left(x^{*}\right)>0$ at the extremum point, then $x^{*}$ is a minimizer for $x$ and the update for Newton's method is

$$
x^{[n+1]}=x^{[n]}-\frac{f^{\prime}\left(x^{[n]}\right)}{f^{\prime \prime}\left(x^{[n]}\right)} .
$$

It can also be used in a multidimensional case and then a step in Newton's method can be written as

$$
\mathbf{x}^{[n+1]}=\mathbf{x}^{[n]}-\left[\mathbf{f}^{\prime}\left(\mathbf{x}^{[n]}\right)\right]^{-1} \mathbf{f}\left(\mathbf{x}^{[n]}\right)
$$

It can also be used to minimize functions of multiple variables. Suppose $g(\mathbf{x})$ is a function of multiple variable then there is an extremum for $g(\mathbf{x})$ where $\frac{\partial g}{\partial \mathbf{x}}=0$. So we define $\mathbf{f}(\mathbf{x})=\frac{\partial \mathbf{g}}{\partial \mathbf{x}}$ and find the zero of $\mathbf{f}$. In order to derive Newton's method for this minimization we perform a Taylor series expansion of $g(\mathbf{x})$ about the point $g\left(\mathbf{x}^{[n]}\right)$ as

$$
g(\mathbf{x})=\mathbf{g}\left(\mathbf{x}^{[\mathbf{n}]}\right)+\left(\mathbf{x}-\mathbf{x}^{[\mathbf{n}]}\right) \nabla \mathbf{g}+\frac{\mathbf{1}}{\mathbf{2}}\left(\mathbf{x}-\mathbf{x}^{[\mathbf{n}]}\right)^{\mathbf{T}} \nabla^{\mathbf{2}} \mathbf{g}\left(\mathbf{x}-\mathbf{x}^{[\mathbf{n}]}\right)+\text { higher order terms. }
$$

Here,

$$
\nabla g=\frac{\partial g}{\partial \mathbf{x}}=\left[\begin{array}{c}
\frac{\partial g}{\partial x_{1}} \\
\frac{\partial g}{\partial x_{2}} \\
\vdots \\
\frac{\partial g}{\partial x_{n}}
\end{array}\right]
$$

and

$$
\nabla^{2} g=\left(\begin{array}{ccc}
\frac{\partial^{2} g}{\partial x_{1} \partial x_{1}} & \frac{\partial^{2} g}{\partial x_{1} \partial x_{2}} & \cdots \frac{\partial^{2} g}{\partial x_{1} \partial x_{n}} \\
\frac{\partial^{2} g}{\partial x_{2} \partial x_{1}} & \frac{\partial^{2} g}{\partial x_{2} \partial x_{2}} & \cdots \frac{\partial^{2} g}{\partial x_{2} \partial x_{n}} \\
\vdots & & \\
\frac{\partial^{2} g}{\partial x_{n} \partial x_{1}} & \frac{\partial^{2} g}{\partial x_{n} \partial x_{2}} & \cdots \frac{\partial^{2} g}{\partial x_{n} \partial x_{n}}
\end{array}\right)
$$


The matrix $\nabla^{2} g$ is also known as the Hessian of $g$ and if the Hessian is positive definite then the extremum obtained is the minimum. Ignoring the higher order terms (D-13) its quadratic approximation can be written as

$$
Q=g(\mathbf{x})=\mathbf{g}\left(\mathbf{x}^{[\mathbf{n}]}\right)+\left(\mathbf{x}-\mathbf{x}^{[\mathbf{n}]}\right) \nabla \mathbf{g}+\frac{\mathbf{1}}{\mathbf{2}}\left(\mathbf{x}-\mathbf{x}^{[\mathbf{n}]}\right)^{\mathbf{T}} \nabla^{\mathbf{2}} \mathbf{g}\left(\mathbf{x}-\mathbf{x}^{[\mathbf{n}]}\right) .
$$

Now if the gradient of $(\overline{D-16})$ is taken with respect to $\left(\mathbf{x}-\mathbf{x}^{[n]}\right)$ and equate it to zero, the Newton's equation would be obtained as

$$
\nabla^{2} g \mathbf{x}^{[n]}\left(\mathbf{x}-\mathbf{x}^{[n]}\right)+\nabla g \mathbf{x}^{[n]}=0 .
$$

Solving this for the minimizing value of $\mathbf{x}$, we get

$$
\mathbf{x}^{[n+1]}=\mathbf{x}^{[n]}-\left(\nabla^{2} g \mathbf{x}^{[n]}\right)^{-1} \nabla g \mathbf{x}^{[n]} .
$$

We can write $(\bar{D}-18)$ as

$$
\mathbf{x}^{[n+1]}=\mathbf{x}^{[n]}-H\left(\mathbf{x}^{[n]}\right)^{-1} \nabla g \mathbf{x}^{[n]}
$$

where $H\left(\mathbf{x}^{[n]}\right)$ is the Hessian. Sometimes the evaluation of the Hessian becomes difficult and impractical and this leads to the Quasi-Newton methods where an approximation of the inverse Hessian is used. In quasi-Newton methods, instead of the true Hessian, an initial matrix $H\left(\mathbf{x}^{[0]}\right)$ is chosen (usually $H\left(\mathbf{x}^{[0]}\right)=I$ ) which is subsequently updated by the formula

$$
H\left(\mathbf{x}^{[n+1]}\right)=H\left(\mathbf{x}^{[n]}\right)+H\left(\mathbf{x}^{[n]}\right)^{u},
$$

where $H\left(\mathbf{x}^{[n]}\right)^{u}$ is the update matrix. Starting with two points $\mathbf{x}^{[n]}$ and $\mathbf{x}^{[n+1]}$, we define $t^{[n]}=\nabla g\left(\mathbf{x}^{[n]}\right)$ and $t^{[n+1]}=\nabla g\left(\mathbf{x}^{[n+1]}\right)$. Also, let $p^{[n]}=\mathbf{x}^{[n+1]}-\mathbf{x}^{[n]}$, then

$$
t^{[n+1]}-t^{[n]} \approx H\left(\mathbf{x}^{[n]}\right) p^{[n]} .
$$

This can be written as

$$
q^{[n]}=H\left(\mathbf{x}^{[n]}\right) p^{[n]}
$$

if the Hessian is constant and in that case the following condition should also be true.

$$
H^{-1}\left(\mathbf{x}^{[n+1]}\right) q^{[i]}=p^{[i]}, \text { for } 0 \leq i \leq k .
$$

This is called the quasi-Newton condition. The update formulas proposed by Davidon Fletcher-Powell (DFP) and Broyden-Fletcher-Goldfarb-Shanno (BFGS) are two of the widely accepted and used formulas. 